\title{
WestVirginiaUniversity
}

THE RESEARCH REPOSITORY @ WVU

Graduate Theses, Dissertations, and Problem Reports

1998

\section{Local damage analysis of fiber -reinforced polymer matrix composites}

Arsenio Domingo Caceres

West Virginia University

Follow this and additional works at: https://researchrepository.wvu.edu/etd

\section{Recommended Citation}

Caceres, Arsenio Domingo, "Local damage analysis of fiber -reinforced polymer matrix composites" (1998). Graduate Theses, Dissertations, and Problem Reports. 3143.

https://researchrepository.wvu.edu/etd/3143

This Dissertation is protected by copyright and/or related rights. It has been brought to you by the The Research Repository @ WVU with permission from the rights-holder(s). You are free to use this Dissertation in any way that is permitted by the copyright and related rights legislation that applies to your use. For other uses you must obtain permission from the rights-holder(s) directly, unless additional rights are indicated by a Creative Commons license in the record and/ or on the work itself. This Dissertation has been accepted for inclusion in WVU Graduate Theses, Dissertations, and Problem Reports collection by an authorized administrator of The Research Repository @ WVU.

For more information, please contact researchrepository@mail.wvu.edu. 


\title{
LOCAL DAMAGE ANALYSIS OF FIBER REINFORCED POLYMER MATRIX COMPOSITES
}

\author{
by \\ Arsenio Caceres
}

\begin{abstract}
A DISSERTATION
Submitted to

The College of Engineering

at

West Virginia University
\end{abstract}

in partial fulfillment of the requirements

for the degree of

Doctor of Philosophy

in

Mechanical Engineering

Department of Mechanical and Aerospace Engineering Morgantown, West Virginia

1998 


\section{ACKNOWLEDGEMENTS}

First of all, I would like to thank my advisor, Dr. Ever Barbero, for the opportunity he has given me to work in this challenging field of composite materials. His advice and suggestions were always appropriate and constructive to the completion of this degree.

Funding for the first two years was provided by the USDOT/Federal Railroad Administration through Grant/Contract NO: DTFR53-94-G-00039. The Southern Regional Educational Board (SREB) provided the funds for the last year through a scholarship for minority students.

My wife, Cecilia, also deserves a special thank for having placed a parenthesis in her career to come and be with me in Morgantown. She always helped me in many ways.

In moments of need I received emotional and financial support from my parents-in-law, Don Pedro and Doña Aida. God bless their kind hearts.

There were other people who helped to the successful completion of this degree. To all of them: thank you very much for your help. 


\begin{abstract}
While it is widely recognized that failure occurs as a result of damage accumulation, characterization of composite material behavior is currently limited to obtaining the elastic properties and the strength values. With such information, it is not possible to assess the remaining life of a structural component or to estimate the likelihood of failure as a function of the time in service. The present investigation concerns the development of a continuum damage mechanics (CDM) formulation for polymer-matrix composites assuming the damage accumulates in the matrix, fiber, and fiber-matrix interphase regions. A local approach was taken by dividing the analysis of damage in the three phases. The phases may be initially isotropic but become orthotropic as a result of damage. The damage evolution in each phase is orthotropic without any a priori imposition of preferential orientation for the damage or orthotropy of the degraded material properties. Orthotropic damage is represented by a second order symmetric tensor. The development of the degraded constitutive equations is carried out using an equivalent fourth order tensor. Great effort was invested in developing a physically and mathematically consistent representation of damage evolution, which implied significant development of tensor analysis. The identification of material constants is based on accepted simple models of fiber and matrix behavior, thus minimizing the amount of testing required.
\end{abstract}




\section{Contents}

1 INTRODUCTION 1

1.1 Composite materials .......................... 1

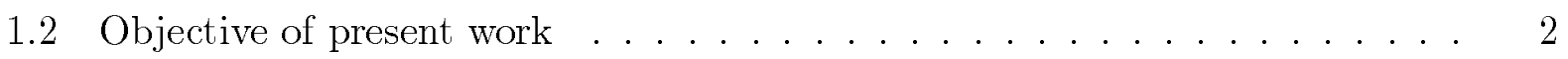

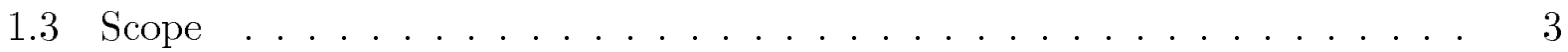

2 DAMAGE MECHANICS $\quad 4$

2.1 Introduction . . . . . . . . . . . . . . . . . 4

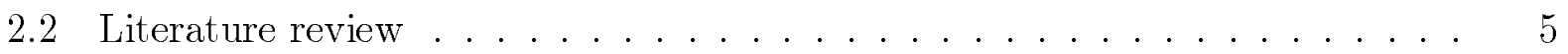

2.3 Measure of damage . . . . . . . . . . . . . . . . 5

2.4 Representative volume element . . . . . . . . . . . . . . 6

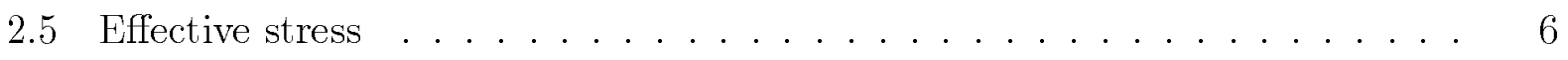

2.6 Critical damage state . . . . . . . . . . . . . . . . 9

2.7 Strain equivalence . . . . . . . . . . . . . . . . 9

2.8 Energy equivalence . . . . . . . . . . . . . . . 10

2.9 Damage effect tensor $M$ and damage tensor $\Omega \ldots \ldots 11$

2.10 Thermodynamic process in damage mechanics . . . . . . . . . . 17

2.11 Damage surface . . . . . . . . . . . . . . . . 18

2.12 Damage evolution . . . . . . . . . . . . . . . . 20

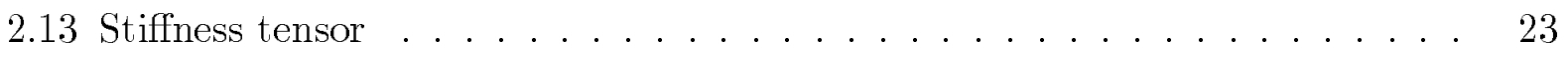

2.14 Summary . . . . . . . . . . . . . . . . . . . 24 
3 DAMAGE IN COMPOSITES $\quad 26$

3.1 Introduction . . . . . . . . . . . . . . . . . . 26

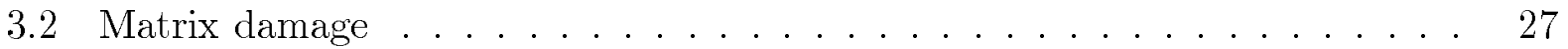

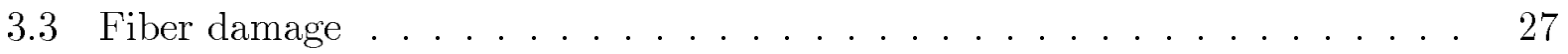

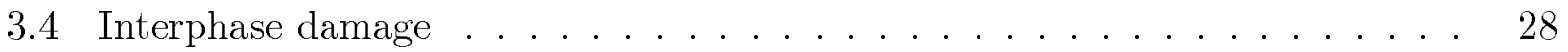

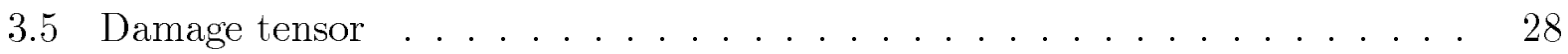

3.6 Micromechanical representation . . . . . . . . . . . . . . . . . 29

3.7 Damage representation . . . . . . . . . . . . . . . . . . . . . 29

3.8 Stress and strain concentration factors $\ldots \ldots \ldots \ldots \ldots$

3.8 .1 Effective configuration . . . . . . . . . . . . . . 31

3.8 .2 Partially damaged configuration . . . . . . . . . . . . 33

3.8 .3 Damaged configuration . . . . . . . . . . . . . . . . 34

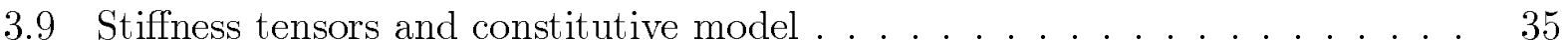

3.9.1 Constitutive model for matrix and fiber . . . . . . . . . . . 36

3.9 .2 Constitutive equation for the composite . . . . . . . . . . 37

3.10 Summary . . . . . . . . . . . . . . . . . . . . 43

4 CONSTANTS DETERMINATION 44

4.1 Introduction . . . . . . . . . . . . . . . . . 44

4.2 Thermodynamic force . . . . . . . . . . . . . . . . . 44

4.3 Hardening parameter and damage surface $\ldots \ldots \ldots \ldots$. . . . . . 47

4.4 Damage evolution law . . . . . . . . . . . . . . . . . . . 49

4.5 Graphical determination of damage parameters $\ldots \ldots \ldots \ldots$

4.5 .1 Kinematic law $X \ldots \ldots \ldots \ldots \ldots \ldots$

4.5.2 Damage multiplier threshold $u_{o} \ldots \ldots \ldots \ldots \ldots$

4.5 .3 Hardening parameter $\kappa \ldots \ldots \ldots \ldots \ldots \ldots \ldots$

4.6 Hardening factor $\eta$ and hardening exponent $\xi \ldots \ldots \ldots \ldots$

4.6 .1 Strain equivalence principle case . . . . . . . . . . . . 56 
$4.6 .2 \quad$ Energy equivalence principle . . . . . . . . . . . . . 57

4.7 Stress-strain curve from model . . . . . . . . . . . . . . . . . . 58

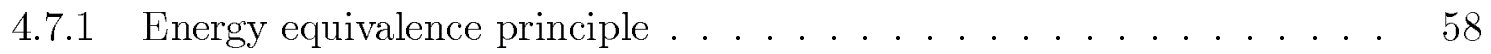

4.8 Application to Janson and Hult model . . . . . . . . . . . . . . . . 59

4.8 .1 Strain equivalence principle $\ldots \ldots \ldots \ldots \ldots$

$4.8 .2 \quad$ Energy equivalence principle . . . . . . . . . . . . . . . 61

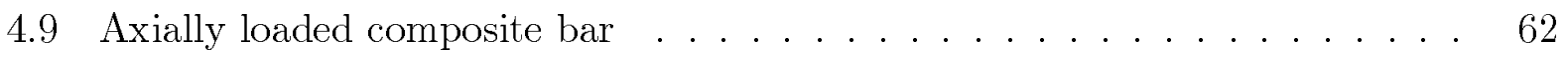

4.9.1 Stress concentration factors in the damaged configuration . . . . . . 63

4.9.2 Determination of in-situ matrix stress-strain curve . . . . . . . . . 65

4.9 .3 Parameter identification . . . . . . . . . . . . . . . 69

4.9 .4 Accuracy of the model . . . . . . . . . . . . . . 70

4.10 Transversely loaded composite bar . . . . . . . . . . . . . . . . 71

4.11 Summary . . . . . . . . . . . . . . . . . . 76

$\begin{array}{lll}5 & \text { FINITE ELEMENT IMPLEMENTATION } & \mathbf{7 7}\end{array}$

5.1 Introduction . . . . . . . . . . . . . . . . . 77

5.2 Damaged stiffness and evolution law $\ldots \ldots \ldots \ldots \ldots \ldots$

5.3 Nodal forces . . . . . . . . . . . . . . . . . . . 78

5.4 Computation of damage for the composite $\ldots \ldots \ldots \ldots$. . . . . 82

6 SUMMARY AND CONCLUSIONS

A Derivatives of $M$ with respect to $\Omega \quad 86$

$\begin{array}{ll}\text { B Tensor Algebra } & 91\end{array}$

B.1 Tensor symmetries . . . . . . . . . . . . . . . . . . . . . 91

B.2 Matrix representation of a tensor $\ldots \ldots \ldots \ldots \ldots \ldots$

B.3 Inner-product tensor multiplication $\ldots \ldots \ldots \ldots \ldots . \ldots \ldots$

B.4 Tensor inversion . . . . . . . . . . . . . . . . . . . . . . . . 94 


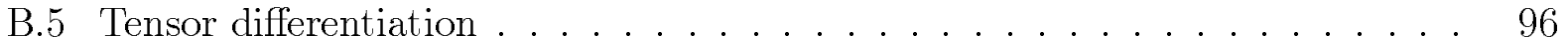

B.5.1 Derivative of a tensor with respect to itself . . . . . . . . . 96

B.5.2 Derivative of the inverse of a tensor with respect to the tensor . . . 96

$\begin{array}{lr}\text { C Minimum square of type } y=a[f(x)]^{b} & \mathbf{9 8}\end{array}$

$\begin{array}{ll}\text { D Elastic stress and strain concentration factors } & 100\end{array}$ 


\section{List of Figures}

$1.1 \quad$ A composite representative volume. . . . . . . . . . . . . . . 1

2.1 Configurations for an axially loaded bar. . . . . . . . . . . . 8

2.2 Loading in damage process. . . . . . . . . . . . . . . . 20

3.1 Matrix, fiber and composite stresses in Representative Volume Element (RVE). 29

3.2 Configurations and phases in damaged composite . . . . . . . . . . 30

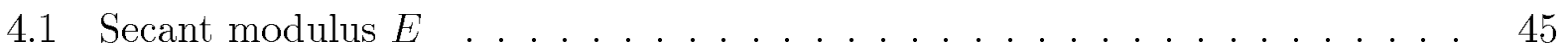

4.2 Determination of $X$. Strain equivalence principle. . . . . . . . . . . . 51

4.3 Determination of $X$. Energy equivalence principle. . . . . . . . . . . 52

4.4 Graphical determination of parameter $u_{o}$. Strain equivalence principle. . . 54

4.5 Graphical determination of hardening parameter $\kappa \ldots \ldots 6$

4.6 Stress-strain curve for a Janson and Hult material. . . . . . . . . . . . . . 60

4.7 Phases and transformation between damage configurations of a composite. $\quad$. 62

4.8 Stress strain curve for fiber T300 (Rosen model). . . . . . . . . . . . . 67

4.9 Stress-strain curves for a matrix, fiber and the composite. . . . . . . . . 68

4.10 Stress-strain curve for matrix and fiber bundle. . . . . . . . . . . 70

4.11 Composite curve obtained from matrix and fiber curve. . . . . . . . . . 71

4.12 Comparison of model and real stress-strain curve for the fibers. . . . . . . . 72

4.13 Bar transversely loaded. . . . . . . . . . . . . . . . . 73

4.14 Quantification of debonding damage. . . . . . . . . . . 73 
4.15 Stress transformations for a transversely loaded bar. . . . . . . . . . . . 74

5.1 Elastic and damaged components of a stress increment. . . . . . . . . . 81

5.2 Configurations for damage computation . . . . . . . . . . . 82 


\section{List of Tables}

4.1 Experimental data for fiber and composite . . . . . . . . . . . . . 69

4.2 Additional data for matrix and fiber bundle . . . . . . . . . . . . . 69

4.3 Tangent modulus at damage onset and hardening parameter . . . . . . . 70

4.4 Values of $\eta$ and $\xi \ldots \ldots \ldots \ldots 71$

4.5 Summary of material parameters . . . . . . . . . . . . 76 


\section{Chapter 1}

\section{INTRODUCTION}

\subsection{Composite materials}

A composite is a material made up of two or more constituents. Usually one phase acts as a reinforcement of a second phase. This reinforcement is usually strong fibers and the phase that it reinforces is called the matrix (see Figure 1.1). The fibers might be carbon, silicon carbide, nylon, or metal whiskers, and the matrix might be a metal, an epoxy resin, ceramics, or rubber.

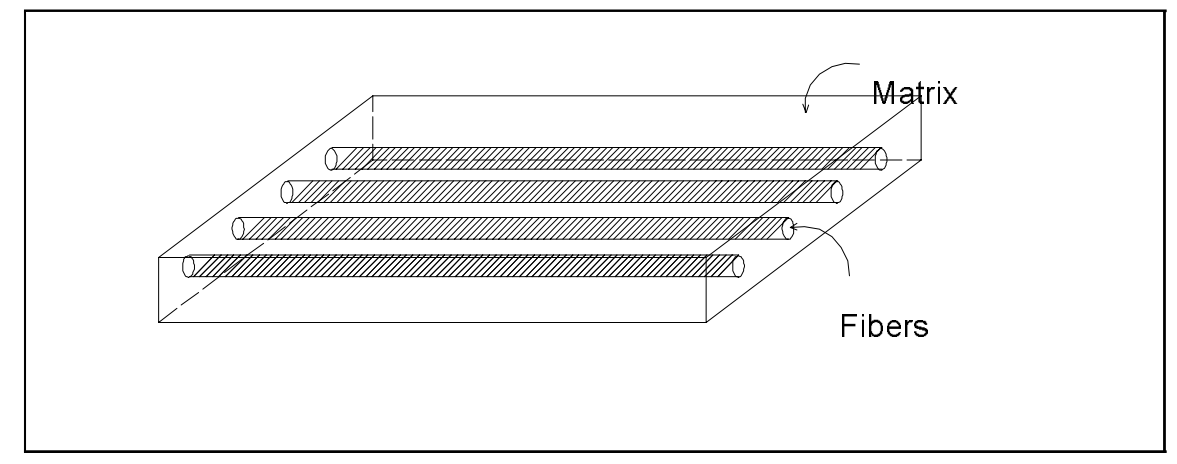

Figure 1.1: A composite representative volume.

The volume ratio $(v)$ is the relation between the volume of one constituent and the total volume. It is generally defined for the fibers: 


$$
v_{f}=\frac{A_{f}}{A}
$$

The introduction of a family of fibers possessing a definite orientation immediately introduces a preferred direction in the material. Thus, even if the constituents are isotropic, the composite itself will initially be anisotropic or transversely isotropic.

Development of composites started in the early 1960s. The development of new materials and applications for composites is accelerating nowadays, especially in applications needing high stiffness/density and strength/density ratio. Originally, composites applications were restricted to specialty areas such as athletic equipment and aerospace structures. Now we are in the verge of an increase in the use of composites for civil engineering applications, for example the recent opening of an all-composite bridge in West Virginia. Other promising applications include all-composite automobile, skins on super/hypersonic atmospheric flight vehicles, hot gas turbine components, heat transfer devices, and space structures. Fiber reinforced plastic (FRP) composites will probably replace conventional materials in many civil engineering structures including buildings, highway bridges, sewage and water treatment facilities, off-shore structure and transportation systems (Barbero and Gangarao [25], [26]).

\subsection{Objective of present work}

The objectives of this work are the following:

- Introduce an anisotropic damage model to capture more accurately the mechanical behavior of brittle matrix composites. This model will include matrix and fiber damage as well as debonding damage.

- Write a Finite Element program to solve numerically the proposed model.

- Formulate a numerical procedure to determine from experimental data the material constants needed for the model.

- Compare the theoretical results with experimental data.

- Solve the problem of the plate with a center hole. 


\subsection{Scope}

A review of damage mechanics is presented in Chapter 2. The equations are presented for the case of a material under damage. Differences between the strain and energy equivalence principle are discussed. The constitutive equations and evolution law are also formulated. A definition of the fourth-order damage tensor $M$ and its relation with the second-order damage tensor $\Omega$ is presented.

The damage mechanics in composite materials is analyzed in Chapter 3. The different modes of damage and their relationship between one another are discussed. The relation among the different phases of the composite material is also described.

The characterization of material constants needed to make use of the model is explained in Chapter 4. Also, the methods to determine these constants from experimental data from composites and fiber are described.

A Finite Element formulation is presented in Chapter 5.

The findings, conclusions and suggestions for further study are presented in Chapter 6. 


\section{Chapter 2}

\section{DAMAGE MECHANICS}

\subsection{Introduction}

From a physical point of view, damage represents discontinuities in the form of microcracks or volume discontinuities in the form of cavities in a material. They are formed as the material undergoes an increasing load.

The objective of damage mechanics is to predict, through mechanical variables, the response of a material in the presence of damage. Damage is initiated at some stress level and it generally increases with increasing stress from the virgin state up to a macroscopic crack initiation or failure. From this point on, the phenomenon is dealt with by fracture mechanics.

Damage is considered a non-reversible process. It can only grow and there is no recovering or healing even as the material unloads. The evolution of damage results in a progressive deterioration of the stiffness matrix in a non-linear, inelastic response. Unlike plasticity, as a result of damage there is no permanent or remaining strains after unloading.

Due to the difficulty associated with measuring damage directly at a microscopic scale numerous damage theories have been proposed, both micromechanical and phenomenological. These different theories try to account for the degree of approximation with which the internal variables describe the important aspects of the macroscopic effects. 


\subsection{Literature review}

The earliest published works on damage mechanics appear to be those by Kachanov [1] and Rabotnov [2] on the application of a continuous damage variable to creep failure of metals under uniaxial loads. The concept was generalized, within the framework of irreversible thermodynamics and extended to ductile fracture and fatigue fracture, for isotropic materials under multiaxial loads by Lemaitre and Chaboche [3], Hult [4], Leckie [5], and Murakami [6], among others.

\subsection{Measure of damage}

Damage can be detected by measuring macroscopic properties of the composite: moduli, electrical conductivity or light scattering, X-ray absorption, or ultrasonic attenuation. The measurement of moduli is particularly attractive because, being the stiffness a fourth order tensor, it offers the possibility of distinguishing and measuring different components of damage.

The damage can be measured by means of a variable, and according to the nature of the damage process that variable can be:

- Scalar representation $D$ : This variable is convenient when the microcrack distribution can be assumed to be approximately isotropic. This, in turn, may occur when the microcrack density is a weak function of the plane orientation and a single scalar defines fully the microcrack distribution. The thermodynamic damage variable $D$ represents the amount of volumetric material damage. The lack of detail in order to model the anisotropy associated with damage is the major weakness of the isotropic theories. An additional, and somewhat related, deficiency is the inability to differentiate between compressive and tensile loading. Due to its simplicity the scalar damage variable representation has been extensively used in the past (Kachanov [9], Lemaitre and Chaboche [10], Hayhurst[11].)

- Vector representation $\rho_{i}$ : In this representation, a crack is totally defined by its area, normal to its surface and the coordinates of the crack center (Davidson and Stevens [27], Krajcinovic and Fonseca [28].)

- Second-order tensor $\Omega_{i j}$ : In a general case the microcrack distribution is characterized by a varying degree of anisotropy. When the crack distribution induces an orthotropic 
phenomenon, the damage variable can be represented by a second-order tensor $\Omega_{i j}$. The orthotropic damage may be induced by the stresses, or be a function of the variations of strength and stiffness with orientation. The principal planes of the damage density is often perpendicular assuming that the state of stress is simple and the loads proportional.

- Fourth-order tensor $M_{i j k l}$ : This is the general case of non-proportional loading where all the trends of the microcrack distribution can be captured.

\subsection{Representative volume element}

A volume defining the minimum size of a matter for which the continuum theories are still valid is called a representative volume element (RVE). It is, therefore, the smallest volume of the material

- that possesses the same material characteristics as the test specimen

- for which the continuum mechanics methods based on the averaged (effective) moduli and volume averages of the phase variables still lead to results of acceptable accuracy.

In general the size of the RVE must satisfy two criteria: it must be large enough to contain a statistically valid sample of constituents, grains, aggregates and defects or other sources of heterogeneity, and yet be small enough to render its mapping on a material point meaningful.

\subsection{Effective stress}

The appearance of discontinuities leads directly to the concept of effective stress, that is, the stress calculated over the section which effectively resists the forces. In the case of a uniaxial tensile load, the nominal or Cauchy stress is computed as (see Figure 2.1)

$$
\sigma=\frac{P}{A}
$$

where $P$ is the axial load applied and $A$ is the cross sectional area. 
If the stress reaches a certain value, damage starts appearing. This stress level is called damage threshold. After this and due to the presence of damage in the cross section, the resisting area is diminished to $A_{e}$, and the effective stress acting on the resisting section is

$$
\bar{\sigma}=\frac{P}{A_{e}}
$$

The damage is defined as the fraction of the cross sectional area that has disappeared.

$$
\Omega=\frac{A-A_{e}}{A}
$$

From Eqns. (2.2), (B.18), and (2.3) a relation between the nominal and effective stress is

$$
\bar{\sigma}=\frac{\sigma}{1-\Omega}
$$

The above relationship is valid for both strain and energy equivalence principles (See sections 2.7 and 2.8). From the above considerations, it is evident that

$$
0 \leq \Omega \leq 1
$$

Here $\Omega=0$ corresponds to the undamaged or virgin material, while $\Omega=1$ corresponds to the breaking of the material into two parts. Furthermore, through a process of instability the failure may occur when $\Omega<1$ (See Section 2.6)

Some adjustment must be made when there is a compressive stress, since some defects close, even though the damage itself remains unchanged. This effect is taken into account through the use of a compression coefficient $C_{n}$ in Section 2.9.

It is helpful to introduce two configurations in a damaged material (see Figure 2.1):

- the damaged configuration where the material is homogenized with the voids and subjected to the nominal stress. 


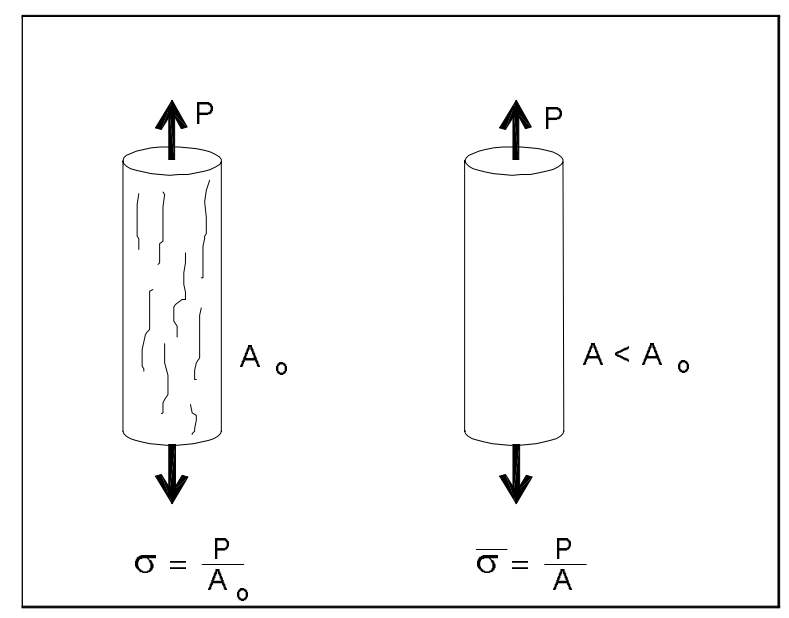

Figure 2.1: Configurations for an axially loaded bar.

- the fictitious undamaged configuration, also called the effective configuration, where all damage has been removed and the material is subjected to the effective stress. A bar over a variable indicates a quantity defined in the effective configuration, e.g. $\bar{\sigma}$.

When the damage is orthotropic a second-order tensor is used to represent it. The effective stress of Eqn. (2.4) takes the form given by Murakami [13]

$$
\bar{\sigma}_{i j}=\left(\delta_{k j}-\Omega_{k j}\right)^{-1} \sigma_{i k}
$$

The effective stress concept states that any deformation behavior, whether uniaxial, multiaxial, elastic, plastic or viscoplastic, of a damaged material is represented by the constitutive laws of the virgin material in which the applied stress $\sigma$ is replaced by the effective stress $\tilde{\sigma}$.

The resulting effective stress given in Eqn. (2.6) is not symmetric and therefore must be symmetrized by an appropriate procedure, one of which is given in Section 2.9.

It may be convenient to express the relation between the stresses in the damaged and effective configurations by means of a fourth-order tensor, related conveniently to the secondorder tensor $\Omega_{i j}$. The transformation represented by Eqn. (2.6) takes the form

$$
\bar{\sigma}_{i j}=M_{i j k l} \sigma_{k l}
$$


The relation between $\Omega_{i j}$ and $M_{i j k l}$ is derived in Section 2.9.

\subsection{Critical damage state}

Usually the limit value of the damage variable is $\Omega=1$. Actually, the failure often occurs earlier when the effective stress of Eqn. (2.4) on the RVE reaches the stress which induces atomic decohesion, $\sigma_{u}$ in uniaxial conditions. Denoting by $\sigma_{R}$ the ultimate stress of a real material with flaws and by $\Omega^{*}$ the critical damage at initiation of rupture, they can be related by

$$
\sigma_{u}=\frac{\sigma_{R}}{1-\Omega^{*}}
$$

out of which the critical damage at rupture or ultimate stress is

$$
\Omega^{*}=1-\frac{\sigma_{R}}{1-\sigma_{u}}
$$

Experiments show that this critical value of damage is not only material-dependent, but also varies with the kind and history of loading. Nevertheless the critical value given by Eqn. (2.9) may be considered a good first approximation.

\subsection{Strain equivalence}

One approach to solve the damage problem is to establish that the strain tensor is the same in the effective and damaged state (see section 2.5)

$$
\bar{\varepsilon}_{i j}=\varepsilon_{i j}
$$

In the damaged state, the elastic relation is given by

$$
\sigma_{i j}=E_{i j k l} \varepsilon_{k l}
$$


and in the effective state

$$
\bar{\sigma}_{i j}=\bar{E}_{i j k l} \varepsilon_{k l}
$$

and by considering Eqn. (2.7) the damaged elastic (unloading) stiffness tensor is

$$
E_{i j k l}=M_{i j m n}^{-1} \bar{E}_{m n k l}
$$

Note that based on this principle, the damaged elastic stiffness tensor $E_{i j k l}$ is not guaranteed to be symmetric.

\subsection{Energy equivalence}

Another approach is to postulate that the elastic energy stored per unit volume are the same in the effective and damaged state. The hypothesis of strain energy equivalence states:

the elastic energy of the damaged material is the same in form as that of an undamaged material except the stress (strain) is replaced by the effective stress (strain)

By the above definition, the elastic energy for both states are

$$
\frac{1}{2} \bar{\sigma}_{i j} \bar{\varepsilon}_{i j}=\frac{1}{2} \sigma_{i j} \varepsilon_{i j}
$$

Again in the damaged state

$$
\sigma_{i j}=E_{i j k l} \varepsilon_{k l}
$$

and in the effective state

$$
\bar{\sigma}_{i j}=\bar{E}_{i j k l} \bar{\varepsilon}_{k l}
$$


The effect of damage in the stress and strains is

$$
\bar{\sigma}_{i j}=M_{i j k l} \sigma_{k l}
$$

By combining Eqns. (2.14) and (2.17);

$$
\bar{\varepsilon}_{i j}=M_{k l i j}^{-1} \varepsilon_{k l}
$$

Again combining Eqns. (2.15), (2.16), (2.17), and (2.18) the effective elastic modulus is found

$$
E_{i j k l}=M_{i j m n}^{-1} \bar{E}_{m n p q} M_{k l p q}^{-1}
$$

Unlike in the strain equivalence principle, one important characteristic of the energy equivalence principle is that the damaged elastic modulus is always symmetric, regardless of the nature of the tensor $M$. The energy equivalence principle is used in this work.

\subsection{Damage effect tensor $M$ and damage tensor $\Omega$}

In this work, a fourth-order damage tensor is used to model damage, as set forth in Eqn. (2.7). Nevertheless, the damage considered is orthotropic, and therefore, representable by a second-order tensor $\Omega_{i j}$. In this section a relation between these two tensors is presented. This relation is based on the principal values of the damage tensor. At the same time the effect of crack closure in compressive and shear stresses is considered.

The principal values of $\Omega_{i j}$ are found by solving the system

$$
\left[\Omega_{i j}-\Omega^{(q)} \delta_{i j}\right] n_{i}^{(q)}=0
$$

combined with the condition

$$
n_{i}^{(q)} n_{i}^{(q)}=1
$$


The roots of (2.20) are given by

$$
\Omega^{(i) 3}-I_{1} \Omega^{(i) 2}+I_{2} \Omega^{(i)}-I_{3}=0
$$

where

$$
\begin{aligned}
I_{1} & =\Omega_{i i} \\
I_{2} & =\frac{1}{2}\left(\Omega_{i i} \Omega_{j j}-\Omega_{i j} \Omega_{j i}\right) \\
I_{3} & =\epsilon_{i j k} \Omega_{1 i} \Omega_{2 j} \Omega_{3 k}
\end{aligned}
$$

Let $T_{i j}$ be the transformation tensor that diagonalizes $\Omega_{i j}$, defined as

$$
[T]=\left[\begin{array}{ccc}
n_{1}^{(1)} & n_{2}^{(1)} & n_{3}^{(1)} \\
n_{1}^{(2)} & n_{2}^{(2)} & n_{3}^{(2)} \\
n_{1}^{(3)} & n_{2}^{(3)} & n_{3}^{(3)}
\end{array}\right]
$$

or

$$
T_{i j}=n_{j}^{(i)}
$$

where the elements $n_{j}^{(i)}$ are the eigenvectors of $\Omega_{i j}$. The damage tensor is not diagonal when represented in material coordinates (1-2-3) following the usual notation for composites. This means that damage is not restricted to three orthogonal planes of cracks aligned with the material orientation (1-2-3) but to three orthogonal planes oriented arbitrarily in space.

The principal values of $\Omega_{i j}$ are therefore given by

$$
\Omega_{i j}^{\prime}=T_{i k} T_{j l} \Omega_{k l}
$$

The Cauchy stress $\sigma_{i j}$, acting on the material orientation, may be transformed into the direction of principal axes of $\Omega_{i j}$ by the same transformation. 


$$
\sigma_{i j}^{\prime}=T_{i k} T_{j l} \sigma_{k l}
$$

Due to the effect of crack closure different types of stresses influence differently on the damage behavior. To take into account this phenomenon the stress tensor $\sigma^{\prime}$ is split into its tensile part $\sigma^{(t)}$, the compressive part $\sigma^{\prime(c)}$, and the shearing part $\sigma^{(s)}$. Using the superposition principle one may write

$$
\sigma_{i j}^{\prime}=\sigma_{i j}^{(t)}+\sigma_{i j}^{(c)}+\sigma_{i j}^{(s)}
$$

or

$$
\begin{aligned}
{\left[\begin{array}{lll}
\sigma_{11} & \sigma_{12} & \sigma_{12} \\
\sigma_{21} & \sigma_{22} & \sigma_{23} \\
\sigma_{31} & \sigma_{32} & \sigma_{33}
\end{array}\right]=} & {\left[\begin{array}{ccc}
\left\langle\sigma_{11}\right\rangle & 0 & 0 \\
0 & \left\langle\sigma_{22}\right\rangle & 0 \\
0 & 0 & \left\langle\sigma_{33}\right\rangle
\end{array}\right]-\left[\begin{array}{ccc}
\left\langle-\sigma_{11}\right\rangle & 0 & 0 \\
0 & \left\langle-\sigma_{22}\right\rangle & 0 \\
0 & 0 & \left\langle-\sigma_{33}\right\rangle
\end{array}\right] } \\
& +\left[\begin{array}{ccc}
0 & \sigma_{12} & \sigma_{12} \\
\sigma_{21} & 0 & \sigma_{23} \\
\sigma_{31} & \sigma_{32} & 0
\end{array}\right]
\end{aligned}
$$

where the brackets $\langle$.$\rangle represent the Heaviside function, defined as$

$$
\begin{aligned}
& \left\langle X_{i j}\right\rangle=1 \text { if } X_{i j}>0 \\
& \left\langle X_{i j}\right\rangle=0 \text { if } X_{i j} \leq 0
\end{aligned}
$$

The effective stress in the principal axes of $\Omega_{i j}$ is given by Eqn. (2.6)

$$
\begin{aligned}
\tilde{\sigma}_{i j}^{\prime}= & \sigma_{i k}^{(t)}\left(\delta_{k j}-\Omega_{k j}^{\prime}\right)^{-1}+\sigma_{i k}^{\prime(c)}\left(\delta_{k j}-C_{n} \Omega_{k j}^{\prime}\right)^{-1} \\
& +\sigma_{i k}^{\prime(s)}\left(\delta_{k j}-C_{s} \Omega_{k j}^{\prime}\right)^{-1}
\end{aligned}
$$

The coefficient $C_{n}$ is introduced to take into account that for compressive stress, voids and cracks may close and therefore the effective resisting area may increase. If the crack 
closes completely $C_{n}=0$. If the crack does not close at all, $C_{n}=1$. In a general case $0 \leq C_{n} \leq 1$. Similarly, $C_{s}$ takes into account that the crack surface may not be perfectly smooth, therefore some amount of shearing force can be transmitted through the crack when the stress is compressive.

The effective stress given by Eqn. (2.31) is not symmetric. Since it is inconvenient to formulate constitutive and evolution equations of damaged materials by using an asymmetric tensor $\tilde{\sigma}_{i j}$, it must be symmetrized in some appropriate way. One possible way is to adopt the symmetric part of the Cartesian decomposition for $\tilde{\sigma}_{i j}$.

$$
\begin{aligned}
\tilde{\sigma}_{i j}^{\prime}= & \frac{1}{2}\left[\sigma_{i k}^{(t)}\left(\delta_{k j}-\Omega_{k j}^{\prime}\right)^{-1}+\left(\delta_{i k}-\Omega_{i k}^{\prime}\right)^{-1} \sigma_{k j}^{(t)}\right] \\
& +\frac{1}{2}\left[\sigma_{i k}^{(c)}\left(\delta_{k j}-C_{n} \Omega_{k j}^{\prime}\right)^{-1}+\left(\delta_{i k}-C_{n} \Omega_{i k}^{\prime}\right)^{-1} \sigma_{k j}^{(c)}\right] \\
& +\frac{1}{2}\left[\sigma_{i k}^{(s)}\left(\delta_{k j}-C_{s} \Omega_{k j}^{\prime}\right)^{-1}+\left(\delta_{i k}-C_{s} \Omega_{i k}^{\prime}\right)^{-1} \sigma_{k j}^{(s)}\right]
\end{aligned}
$$

Taking into account that the damage tensor $\Omega_{i j}^{\prime}$ is diagonal

$$
\begin{aligned}
\tilde{\sigma}_{i j}^{\prime}= & \frac{\sigma_{i j}^{(t)}}{1-\Omega_{i j}^{\prime}}+\frac{\sigma_{i j}^{(c)}}{1-C_{n} \Omega_{i j}^{\prime}}+\frac{1}{2}\left[\frac{\sigma_{i j}^{(s)}}{1-C_{s} \Omega_{i i}^{\prime}}+\frac{\sigma_{i j}^{(s)}}{1-C_{s} \Omega_{j j}^{\prime}}\right] \\
& (i, j \text { not summed })
\end{aligned}
$$

Let the operators $R^{t}, R^{c}$, and $R^{s}$ be defined such that

$$
\begin{aligned}
\sigma_{i j}^{(t)} & =R_{i j k l}^{t} \sigma_{k l}^{\prime} \\
\sigma_{i j}^{\prime(c)} & =R_{i j k l}^{c} \sigma_{k l}^{\prime} \\
\sigma_{i j}^{\prime(s)} & =R_{i j k l}^{s} \sigma_{k l}^{\prime}
\end{aligned}
$$

That is, the tensor $R_{i j k l}$ separates the tensile, compressive and shear components in the principal axes of the damage tensor. It should be noted that the tensor $\mathrm{R}$ is constant as long: as the signs of the normal components of $\sigma_{i j}^{\prime}$ do not change. Also, at any time

$$
R_{i j k l}^{t}+R_{i j k l}^{c}+R_{i j k l}^{s}=I_{i j k l}
$$


where $I_{i j k l}$ is the fourth order identity tensor, defined in Eqn. (B.10).

Now let the tensor $\zeta_{i j k l}$ be defined such that;

$$
\begin{array}{ll}
\zeta_{i j k l}=1 & \text { if } \\
\zeta_{i j k l}=0 & \text { otherwise }
\end{array}
$$

It can be shown that

$$
\begin{aligned}
& R_{i j k l}^{s}=I_{i j k l}-\zeta_{i j k l} \\
& R_{i j k l}^{t}=\zeta_{i j k l}\left\langle\sigma_{k l}^{\prime}\right\rangle . . \text { no summation on } k l \\
& R_{i j k l}^{c}=\zeta_{i j k l}\left\langle-\sigma_{k l}^{\prime}\right\rangle . . \text { no summation on } k l
\end{aligned}
$$

By introducing Eqns. (2.34) and (2.37), Eqn. (2.33) can now be rewritten as

$$
\tilde{\sigma}_{i j}^{\prime}=F_{i j k l} \sigma_{k l}^{\prime}
$$

with

$$
\begin{aligned}
F_{i j k l}= & \frac{\zeta_{i j k l}\left\langle\sigma_{k l}^{\prime}\right\rangle}{1-\Omega_{i j}^{\prime}}+\frac{\zeta_{i j k l}\left\langle-\sigma_{k l}^{\prime}\right\rangle}{1-C_{n} \Omega_{i j}^{\prime}}+\frac{I_{i j k l}-\zeta_{i j k l}}{2}\left(\frac{1}{1-C_{s} \Omega_{i i}^{\prime}}+\frac{1}{1-C_{s} \Omega_{j j}^{\prime}}\right) \\
& (i, j \text { not summed })
\end{aligned}
$$

Considering the symmetry of tensor $F_{i j k l}$, it can be represented by a $6 \times 6$ matrix as follows 


$$
\begin{gathered}
{[F]=\left[\begin{array}{ccc}
\frac{1}{1-\left(\left\langle\sigma_{11}^{\prime}\right\rangle+C_{n}\left\langle-\sigma_{11}^{\prime}\right\rangle\right) \Omega_{11}^{\prime}} & 0 & 0 \\
0 & \frac{1}{1-\left(\left\langle\sigma_{22}^{\prime}\right\rangle+C_{n}\left\langle-\sigma_{22}^{\prime}\right\rangle\right) \Omega_{22}^{\prime}} & 0 \\
0 & 0 & \frac{1}{1-\left(\left\langle\sigma_{33}^{\prime}\right\rangle+C_{n}\left\langle-\sigma_{33}^{\prime}\right\rangle\right) \Omega_{33}^{\prime}} \\
0 & 0 & 0 \\
0 & 0 & 0 \\
0 & 0 & 0 \\
0 & 0 & 0 \\
0 & 0 & 0 \\
0 & 0 & 0 \\
0 & 0 & \frac{1}{4}\left(\frac{1}{1-C_{s} \Omega_{33}^{\prime}}\right) \\
0 & \left.\frac{1}{1-C_{s} \Omega_{22}^{\prime}}+\frac{1}{1-C_{s} \Omega_{33}^{\prime}}\right) & 0 \\
\frac{1}{4}\left(\frac{1}{1-C_{s} \Omega_{11}^{\prime}}+\frac{1}{1-C_{s} \Omega_{22}^{\prime}}\right)
\end{array}\right]}
\end{gathered}
$$

and considering Eqn. (2.27)

$$
\tilde{\sigma}_{i j}^{\prime}=F_{i j k l} T_{k m} T_{l n} \sigma_{m n}
$$

This tensor is transformed back to its original material coordinates

$$
\tilde{\sigma}_{p q}=T_{p i} T_{q j} F_{i j k l} T_{k m} T_{l n} \sigma_{m n}
$$

Now considering the fourth order damage tensor $M_{i j k l}$, which relates the Cauchy and effective stresses and whose expression is given by Eqn. (2.7), it can be seen by comparison with equation (2.42) that the components of $M_{i j k l}$ must be

$$
M_{i j k l}=T_{i m} T_{j n} F_{m n p q} T_{p k} T_{q l}
$$




\subsection{Thermodynamic process in damage mechanics}

The classical way to formulate the dissipative phenomena like plasticity and damage is to postulate the existence of energy potentials from which the state laws and kinetic constitutive equations can be derived. Two potentials can be introduced within the framework of the Static Kinetic Coupling Theory (Lemaitre, [35]).

- The state potential, written as a function of the state variables. It defines the state laws and the variables associated with the state variable used to describe the power involved in each physical process.

- The potential of dissipation written with the associated variables accounts for the kinetic laws of evolution of the flux of dissipative variables.

Within the hypothesis of small strains and small displacements, $\Omega_{i j}$ is the damage variable which is associated with the thermodynamic force $\bar{Y}_{i j}$. Using the Helmhotz free energy function $\Psi$ from which the state laws are derived, the expression for $\bar{Y}_{i j}$ is

$$
\bar{Y}=\rho \frac{\partial \Psi}{\partial \Omega}
$$

In the absence of plastic deformations, the Helmotz free function can be written as

$$
\Psi=\frac{1}{2} \sigma_{i j} E_{i j k l}^{-1} \sigma_{k l}
$$

In order to work with a positive quantity, let

$$
Y=-\bar{Y}
$$

From Eqn. (2.44) the thermodynamic force is given in terms of the damaged elastic stiffness and Cauchy stress as

$$
Y_{m n}=\frac{1}{2} \sigma_{i j} \frac{\partial E_{i j k l}^{-1}}{\partial \Omega_{m n}} \sigma_{k l}
$$


or using Eqn. (2.19), they are given in terms of the initial elastic stiffness

$$
Y_{m n}=\frac{1}{2} \sigma_{i j}\left(\frac{\partial M_{p q i j}}{\partial \Omega_{m n}} M_{r s k l}+M_{p q i j} \frac{\partial M_{r s k l}}{\partial \Omega_{m n}}\right) \bar{E}_{p q r s}^{-1} \sigma_{k l}
$$

\subsection{Damage surface}

Under the assumptions of this work, the fibers are elastic and the matrix undergoes brittle damage. An anisotropic damage model is presented to account for non-proportional loading. This can be accomplished by expressing the damage surface in terms of a tensorial hardening parameter $H_{i j}$. The damage criterion is of the form suggested by Mroz [8]

$$
g=g(Y, H)
$$

The general form of this relation can be formulated by requiring that $g$ be an isotropic function of $Y_{i j}$ and $H_{i j}$, such as

$$
g=H_{i j}^{-1} H_{j k}^{-1} Y_{k l} Y_{l i}-1
$$

A particular form of the hardening tensor $H_{i j}$ for orthotropic materials is given by Voyiadjis and Park [7], as a generalization of the anisotropic model proposed by Stumvol and Swoboda [30]

$$
H_{i j}=\left(u_{i k}\right)^{1 / 2} \Omega_{k l}\left(u_{l i}\right)^{1 / 2}+V_{i j}
$$

where the hardening and the hardening threshold tensors $H_{i j}$ and $V_{i j}$ are here defined for orthotropic materials as

$$
u_{i j}=\left[\begin{array}{ccc}
u_{1}^{0}+\lambda_{1} \eta_{1}\left(\frac{\kappa}{\lambda_{1}}\right)^{\xi_{1}} & 0 & 0 \\
0 & u_{2}^{0}+\lambda_{2} \eta_{2}\left(\frac{\kappa}{\lambda_{2}}\right)^{\xi_{2}} & 0 \\
0 & 0 & u_{3}^{0}+\lambda_{3} \eta_{3}\left(\frac{\kappa}{\lambda_{3}}\right)^{\xi_{3}}
\end{array}\right]
$$


and

$$
V_{i j}=\left[\begin{array}{ccc}
\lambda_{1} v_{1}^{2} & 0 & 0 \\
0 & \lambda_{2} v_{2}^{2} & 0 \\
0 & 0 & \lambda_{3} v_{3}^{2}
\end{array}\right]
$$

The material constants $u_{i}^{0}, \eta_{i}, \xi_{i}$, and $v_{i}$ are obtained with experimental data. The damage multiplier threshold values $u_{i}^{0}$ are related to the slope of the stress-strain curve at the onset of damage as explained in Section 4.5.2. The hardening factor $\eta$ is related to the size of the stress-strain curve and the hardening exponent $\xi$ is related to its shape. They can be determined with the use of the stress-strain curve as explained in Section 4.6. The terms $\lambda_{i}$ are the Lame constants obtained from the elastic stiffness tensor of the material. The terms $v_{i}$ are related to the damage threshold and they can be found at the stress level where the material starts exhibiting non-linearity as

$$
v_{i}=\frac{\sigma_{i 0}}{\sqrt{E_{i} \lambda_{i}}}
$$

from three uniaxial tests.

The scalar hardening parameter $\kappa$ is given by

$$
\kappa=\int_{0}^{t} Y_{i j} \dot{\Omega}_{i j} d t
$$

From the definition of the thermodynamic force $Y_{i j}$ given by Eqn. (2.44), the hardening parameter $\kappa$ can also be expressed as:

$$
\kappa=|\Psi|_{\Omega}-|\Psi|_{\Omega=0}
$$

It must be noticed that $\kappa$ can only increase and that the increase must be due to an increase of damage. Any other type of variation will not produce a variation of $\kappa$ in Eqn. $(2.56)$.

Eqn. (2.49) defines a surface, in the $Y$ space, representing the limit of the undamaged state. A damaging state is any state that satisfies the condition $g=0$. 


\subsection{Damage evolution}

The term damage evolution is commonly used to designate a non-linear deformation process during which three different phenomena take place: nucleation of microcracks, growth of the already existing microcracks, and their coalescence into a macrodefect (macrocrack, microcrack bands, etc.). An incremental analysis, based on the minimization of the dissipative energy of the system, is performed here to establish the damage evolution law.

In an increment of the thermodynamic conjugate force $Y_{i j}$, the following four situations can be distinguished (See Figure 2.2)

$$
\begin{array}{ll}
g<0 & \text { loading or unloading from an elastic state } \\
g=0 & \frac{\partial g}{\partial Y_{i j}} d Y_{i j}<0 \text { elastic unloading } \\
g=0 & \frac{\partial g}{\partial Y_{i j}} d Y_{i j}=0 \text { neutral loading } \\
g=0 & \frac{\partial g}{\partial Y_{i j}} d Y_{i j}>0 \text { loading from a damaging state }
\end{array}
$$

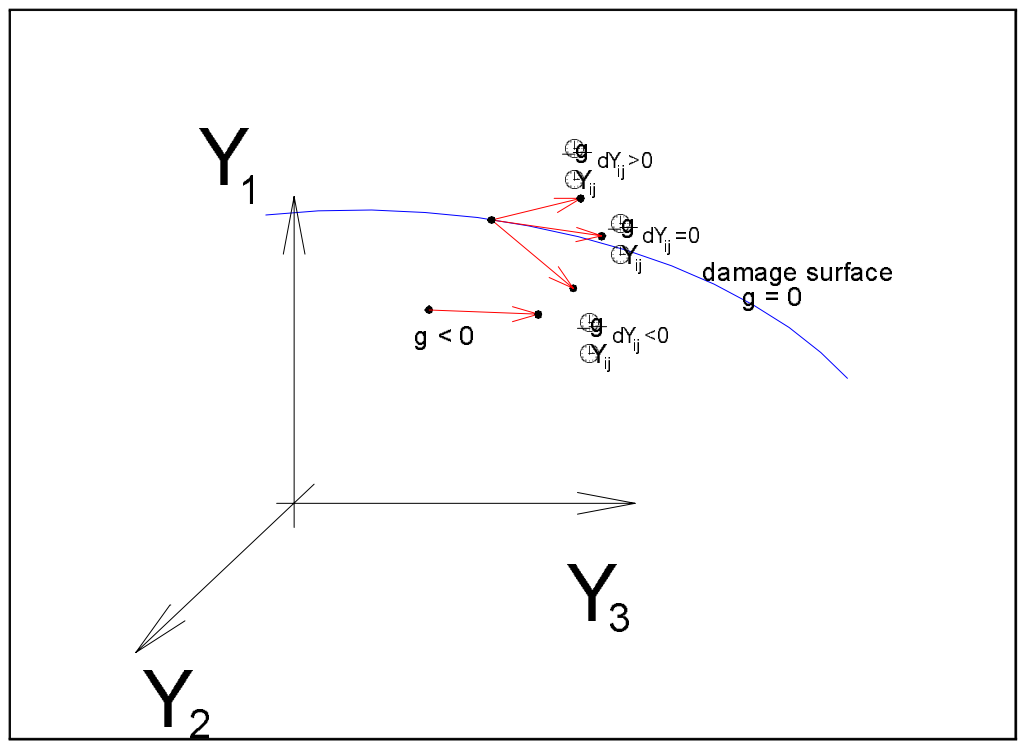

Figure 2.2: Loading in damage process.

Since $d \Omega_{i j}=0$ for the first three cases, the loading from a damaging state is the only 
non-trivial case to be considered. Two principles of dissipative phenomena are considered for this case:

- the flow rule which states that any increment in damage is perpendicular to the damage surface; that is

$$
d \Omega_{i j}=d \Lambda \frac{\partial g}{\partial Y_{i j}}
$$

where $\frac{\partial g}{\partial Y_{i j}}$ is the gradient of the damage surface $g$, and therefore perpendicular to $g$, and $d \Lambda$ is a scalar.

- the consistency condition which states that after an increment of damage, the element must be again on the damage surface

$$
d g=0
$$

By considering Eqn. (2.50) the consistency condition is

$$
d g=\frac{\partial g}{\partial H_{i j}} d H_{i j}+\frac{\partial g}{\partial Y_{i j}} d Y_{i j}=0
$$

From Eqn. (2.48) the thermodynamic force conjugate to damage is a function $Y=$ $Y(\sigma, \Omega)$ and this yields

$$
d Y_{i j}=\frac{\partial Y_{i j}}{\partial \sigma_{m n}} d \sigma_{m n}+\frac{\partial Y_{i j}}{\partial \Omega_{m n}} d \Omega_{m n}
$$

The hardening tensor (Eqns. (2.51), and (2.52)) is $H=H(\Omega, \kappa)$, resulting in

$$
d H_{i j}=\frac{\partial H_{i j}}{\partial \Omega_{m n}} d \Omega_{m n}+\frac{\partial H_{i j}}{\partial \kappa} d \kappa
$$


By looking at (2.55), the increment in the scalar hardening parameter is

$$
d \kappa=\frac{\partial \kappa}{\partial \sigma_{m n}} d \sigma_{m n}+Y_{m n} d \Omega_{m n}
$$

Now, considering the expression for $\kappa$ given by Eqn. (2.56)

$$
\kappa=\frac{1}{2} \sigma_{i j} \hat{E}_{i j k l}^{-1} \sigma_{k l}-|\Psi|_{\Omega=0}
$$

differentiating Eqn. (2.64) and considering Eqn. (2.19)

$$
\frac{\partial \kappa}{\partial \sigma_{m n}}=\frac{1}{2} M_{p q i j} E_{p q r s}^{-1} M_{r s k l}\left(J_{i j m n} \sigma_{k l}+\sigma_{i j} J_{k l m n}\right)
$$

Introducing Eqns. (2.58), (2.61), (2.62), and (2.63) into (2.60) and solving for the scalar parameter $d \Lambda$

$$
d \Lambda=-\frac{\left(\frac{\partial g}{\partial H_{m n}} \frac{\partial H_{m n}}{\partial \kappa} \frac{\partial \kappa}{\partial \sigma_{k l}}+\frac{\partial g}{\partial Y_{m n}} \frac{\partial Y_{m n}}{\partial \sigma_{k l}}\right) d \sigma_{k l}}{\left[\frac{\partial g}{\partial H_{m n}}\left(\frac{\partial H_{m n}}{\partial \Omega_{k l}}+\frac{\partial H_{m n}}{\partial \kappa} Y_{k l}\right)+\frac{\partial g}{\partial Y_{m n}} \frac{\partial Y_{m n}}{\partial \Omega_{k l}}\right] \frac{\partial g}{\partial Y_{k l}}}
$$

and using the flow rule (2.58), the increment of damage is

$$
d \Omega_{i j}=-\frac{\left(\frac{\partial g}{\partial H_{m n}} \frac{\partial H_{m n}}{\partial \kappa} \frac{\partial \kappa}{\partial \sigma_{k l}}+\frac{\partial g}{\partial Y_{m n}} \frac{\partial Y_{m n}}{\partial \sigma_{k l}}\right) d \sigma_{k l}}{\left[\frac{\partial g}{\partial H_{m n}}\left(\frac{\partial H_{m n}}{\partial \Omega_{k l}}+\frac{\partial H_{m n}}{\partial \kappa} Y_{k l}\right)+\frac{\partial g}{\partial Y_{m n}} \frac{\partial Y_{m n}}{\partial \Omega_{k l}}\right] \frac{\partial g}{\partial Y_{k l}}} \frac{\partial g}{\partial Y_{i j}}
$$

or

$$
d \Omega_{i j}=X_{i j k l} d \sigma_{k l}
$$

where 


$$
X_{i j k l}=-\frac{\left(\frac{\partial g}{\partial H_{m n}} \frac{\partial H_{m n}}{\partial \kappa} \frac{\partial \kappa}{\partial \sigma_{k l}}+\frac{\partial g}{\partial Y_{m n}} \frac{\partial Y_{m n}}{\partial \sigma_{k l}}\right) \frac{\partial g}{\partial Y_{i j}}}{\left[\frac{\partial g}{\partial H_{m n}}\left(\frac{\partial H_{m n}}{\partial \Omega_{k l}}+\frac{\partial H_{m n}}{\partial \kappa} Y_{k l}\right)+\frac{\partial g}{\partial Y_{m n}} \frac{\partial Y_{m n}}{\partial \Omega_{k l}}\right] \frac{\partial g}{\partial Y_{k l}}}
$$

or in terms of the increment of strain

$$
d \Omega_{i j}=X_{i j k l} D_{k l m n} d \varepsilon_{m n}=Z_{i j m n} d \varepsilon_{m n}
$$

\subsection{Stiffness tensor}

As in the case of plasticity the instantaneous stiffness tensor varies at each step of the

loading process, and it can be computed by using the following equations. Differentiating Eqns. (2.17) and (2.18)

$$
\begin{aligned}
d \bar{\sigma}_{i j} & =d M_{i j k l} \sigma_{k l}+M_{i j k l} d \sigma_{k l} \\
d \bar{\varepsilon}_{i j} & =d M_{k l i j}^{-1} \varepsilon_{k l}+M_{k l i j}^{-1} d \varepsilon_{k l}
\end{aligned}
$$

Since $M_{i j k l}$ is a function of $\Omega_{m n}$ one can write

$$
\begin{aligned}
d M_{i j k l} & =\frac{\partial M_{i j k l}}{\partial \Omega_{m n}} d \Omega_{m n} \\
d M_{i j k l}^{-1} & =\frac{\partial M_{k l i j}^{-1}}{\partial \Omega_{m n}} d \Omega_{m n}
\end{aligned}
$$

and with Eqn. (2.68)

$$
\begin{aligned}
d M_{i j k l} & =\frac{\partial M_{i j k l}}{\partial \Omega_{m n}} X_{m n p q} d \sigma_{p q} \\
d M_{i j k l}^{-1} & =\frac{\partial M_{k l i j}^{-1}}{\partial \Omega_{m n}} X_{m n p q} d \sigma_{p q}
\end{aligned}
$$

The elastic relations in the undamaged and damaged configurations are, respectively 


$$
\begin{aligned}
\bar{\sigma}_{i j} & =\bar{E}_{i j k l} \bar{\varepsilon}_{k l} \\
\sigma_{i j} & =E_{i j k l} \varepsilon_{k l}
\end{aligned}
$$

The incremental relations in the undamaged and damaged configurations are, respectively

$$
\begin{aligned}
d \bar{\sigma}_{i j} & =\bar{E}_{i j k l} d \bar{\varepsilon}_{k l} \\
d \sigma_{i j} & =D_{i j k l} d \varepsilon_{k l}
\end{aligned}
$$

By substituting Eqns. (2.71), together with Eqn. (2.73) into Eqn. (2.75), the following expression is obtained

$$
\frac{\partial M_{i j k l}}{\partial \Omega_{m n}} X_{m n r s} \sigma_{k l}+M_{i j r s}-\bar{E}_{i j k l} \frac{\partial M_{m n k l}^{-1}}{\partial \Omega_{p q}} X_{p q r s} E_{m n t u}^{-1} \sigma_{t u}=\bar{E}_{i j k l} M_{m n k l}^{-1} D_{m n r s}^{-1}
$$

Let the $N_{i j r s}$ be defined as

$$
N_{i j r s}=\frac{\partial M_{i j k l}}{\partial \Omega_{m n}} X_{m n r s} \sigma_{k l}+M_{i j r s}-\bar{E}_{i j k l} \frac{\partial M_{m n k l}^{-1}}{\partial \Omega_{p q}} X_{p q r s} E_{m n t u}^{-1} \sigma_{t u}
$$

Solving Eqn. (2.77) for $D_{i j k l}$ and using Eqn. (2.78) the damaged stiffness tensor is

$$
D_{i j k l}=N_{i j m n}^{-1} \bar{E}_{m n p q} M_{k l p q}^{-1}
$$

\subsection{Summary}

The most important equations for the development of a complete damage model have been presented in this chapter. The uniaxial case was used to introduce the concepts of effective stress and the damaged and effective configurations. Two approaches to establish the relationship between configurations were presented: the strain equivalence approach and the energy equivalence approach. The stresses in both configurations were related by means of 
a second order damage tensor $\Omega_{i j}$ and a fourth-order damage tensor $M_{i j k l}$. A relationship between these two tensors was derived. The effect of crack closure was also accounted for.

A dissipative potential was proposed to obtain the thermodynamic force conjugate to the damage variable. The concept of a limiting state of stress for which damage evolves was presented by means of a damage surface.

The principle of minimization of the dissipative energy was used to derive the incremental damage evolution law in terms of the increment of stress. To complete the model the stiffness tensor was determined in terms of the damage tensor. 


\section{Chapter 3}

\section{DAMAGE IN COMPOSITES}

\subsection{Introduction}

The most common types of damage in composite materials are:

- Fiber breakage

- Fiber/matrix debonding

- Matrix cracking

- Fiber kinking

- Radial cracks of fibers (for large diameter fibers)

The methods that researchers use to analyze composite materials generally fall into two categories:

- Continuum concept. The composite system is treated as a whole continuum and the equations of anisotropic elasticity are used in the analysis (Arnold and Kruch [12], Talreja [15], Christensen [16]). This method relies heavily on phenomenological datafitting to adjust the many empirical constants needed.

- Micromechanical models with averaging procedures and homogenization. The composite is considered to be composed of a number of phases, and local equations are 
formulated for these phases. A suitable homogenization procedure is then used to deduce the overall response of the composite system from the local analysis (Hill [17], Dvorak and Bahei-El-Din [18]). This is the approach followed in the present work.

For example, Dvorak \& Bahei-El-Din [18] employed a technique proposed initially by Hill [17] to analyze the elastoplastic behavior of fiber-reinforced composites with elastic fibers and elastoplastic matrix. However, no damage effects were introduced in the constitutive equations.

The approach taken in the present work is to consider the damage variable at the constituent level, that is fiber and matrix. The individual constituent response is assembled to yield the composite response. Then, any composite type of damage is added to obtain the overall damage response. Three types of damage are considered next.

\subsection{Matrix damage}

The matrix undergoes brittle damage, that is, a crack is initiated without a large amount of plastic strain. The brittle damage may be in the form of voids, and microcracks, possibly influenced by other damage modes. Eventually, these microdefects become a macrocrack, as seen in experimental tests.

\subsection{Fiber damage}

The primary factor contributing to the complexity of the fiber breakage in composites is the variability of the fiber strength. A consequence of this behavior is that not all fibers will be stressed to their maximum value at the same time. Also, the fibers that break earlier will cause perturbations in the stress fields resulting in localized high interphase shear stresses, and in stress concentrations in adjacent fibers.

At each local fiber break, several possible events may occur. In the neighborhood of the fiber break the local stresses are highly non-uniform. This may result in a crack propagating along the fiber interphase or across the composite. In the former case the fibers may separate from the composite after breaking and the composite material may be no stronger than a dry bundle of fibers. In the second case, the composite fail due to a propagating normal crack or due to a fiber break propagation and the strength of the composite may no be greater 
than that of the weakest fiber (weakest link failure). If the matrix and interphase properties are of sufficient strength and toughness to prevent or arrest these failure mechanisms, then continued load increase will produce new fiber failures at other locations in the material, resulting in a statistical accumulation of internal damage (fiber break propagation failure, Kelly and Barbero [32]).

\subsection{Interphase damage}

Since the fibers act as reinforcement, a major proportion of the load must be carried by them. However the load is usually applied to the matrix, and so must be transferred to the fibers through adhesion or friction. This gives rise to a complex state of stresses and strains in both constituents. Fiber debonding has been analyzed by Needleman [19], Tvergaard [20], and Aboudi [21]. Debonding damage can be modeled as a loss of displacement continuity at the interphase by introducing a thin elastic film as the flexible interphase. The shear stress in the film is taken to be proportional to the relative tangential displacement at the interphase, and the normal stress is proportional to the relative normal displacement. The modeling is therefore reduced to the determination of two parameters that represent the degree of decohesion in the normal and tangential directions.

\subsection{Damage tensor}

Each of the damage modes in a composite material can be represented by a damage tensor. The information on each separate mode is not relevant unless they are assembled to obtain an overall measure of the damage in the composite. In this work, the three damage modes are represented by separate damage tensors. These three damage tensors are:

- $M^{m}$ reflecting all types of damage that can develop in the matrix, mainly matrix cracking.

- $M^{f}$ reflecting all types of damage that can develop in the fibers, mainly fiber breakage.

- $M^{d}$ representing the debonding or interphase damage developed between fibers and matrix. 


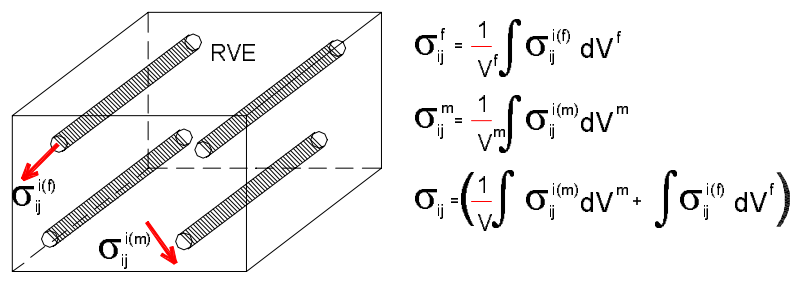

Figure 3.1: Matrix, fiber and composite stresses in Representative Volume Element (RVE).

\subsection{Micromechanical representation}

The micromechanical model represents the composite as an array of fibers allowing the analysis of a RVE rather than the entire composite. An average of the matrix stresses and

strains are taken as the RVE matrix stress $\sigma_{i j}^{m}$. Similarly, the RVE fiber stress $\sigma_{i j}^{f}$ is found. The average composite strain is the volumetric average of the matrix and fiber RVE strains. Likewise, the average composite stress is the volumetric average of the matrix and fiber RVE stresses (see Section 3.8.)

\subsection{Damage representation}

The different phases and damage modes in a composite may be represented at any time by a network (Figure 3.2). The nodes of this network are connected through transformation laws according to the theoretical principles adopted. Three phases exist in a composite: matrix, fiber, and composite. Likewise, three damage modes are considered in this work: matrix cracking, fiber breaking, and fiber-matrix debonding or interphase damage. Three configurations are needed to capture all the damage modes.

- Effective configuration (EC): It is the configuration where all damage has been removed. The constituents are in their virgin state. Due to the removal of damage, the matrix and fiber volume fractions are different from the original.

- Partially damaged configuration (PDC): The matrix and fiber are damaged but there is no debonding damage. It is assumed that the area of the damaged material remains 


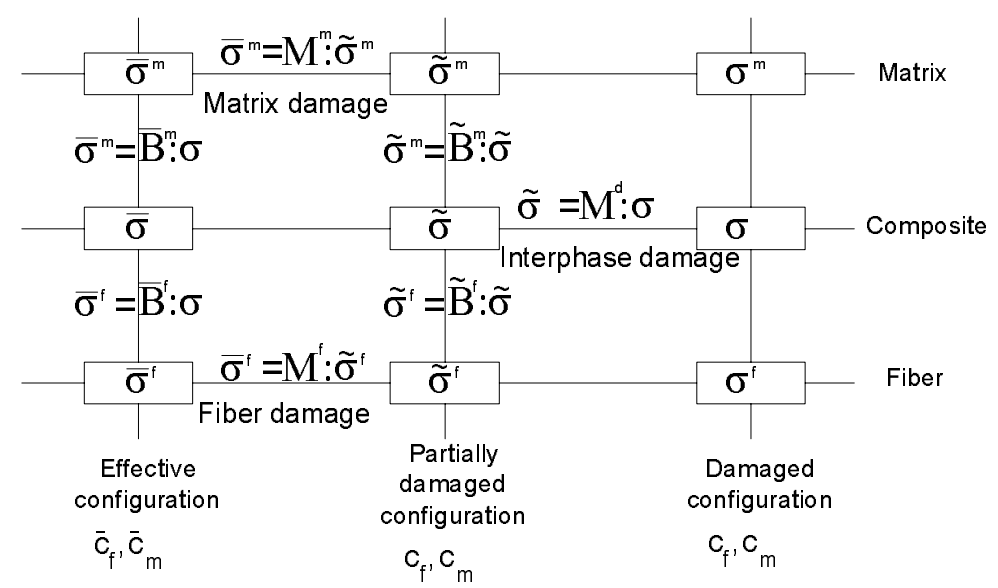

Figure 3.2: Configurations and phases in damaged composite

the same as the original; the matrix and fiber volume fractions are the same as the original. A tilde $\left(^{\sim}\right)$ over a variable indicates a quantity defined in the PDC, e.g. $\tilde{\sigma}$.

- Damaged configuration (DC): It is the real configuration where the composite damage has been added to the PDC. Assuming the composite damage does not influence the volume fractions, the matrix and fiber volume fractions are the same as the original.

Each node of the network represents a configuration and a phase. The matrix and fiber phases are connected to the composite phase through stress concentration factors. These may be different for each configuration. The configurations are connected to each other through a damage tensor. Between the EC and PDC the matrix and fiber stresses are related to their virgin states through the following equation

$$
\begin{aligned}
& \bar{\sigma}_{i j}^{m}=M_{i j k l}^{m} \tilde{\sigma}_{k l}^{m} \\
& \bar{\sigma}_{i j}^{f}=M_{i j k l}^{f} \tilde{\sigma}_{k l}^{f}
\end{aligned}
$$

The overall effective stress is related to the local effective stresses by making use of the micromechanical model such that

$$
\bar{\sigma}_{i j}=\bar{c}^{m} \bar{\sigma}_{i j}^{m}+\bar{c}^{f} \bar{\sigma}_{i j}^{f}
$$


The relation between the strains are

$$
\begin{aligned}
& \bar{\varepsilon}_{i j}^{m}=M_{k l i j}^{-m} \hat{\varepsilon}_{k l}^{m} \\
& \bar{\varepsilon}_{i j}^{f}=M_{k l i j}^{-f} \hat{\varepsilon}_{k l}^{f}
\end{aligned}
$$

Similarly, the micromechanical model yields a relationship between the overall strain and the local strains such that

$$
\bar{\varepsilon}_{i j}=\bar{c}^{m} \bar{\varepsilon}_{i j}^{m}+\bar{c}^{f} \bar{\varepsilon}_{i j}^{f}
$$

Between the PDC and DC all kinds of interphase damage are included (debonding, delamination, etc.) The relation of stresses and strains are

$$
\begin{aligned}
& \tilde{\sigma}_{i j}=M_{i j k l}^{d} \sigma_{k l} \\
& \tilde{\varepsilon}_{i j}=M_{k l i j}^{-d} \varepsilon_{k l}
\end{aligned}
$$

If the stress all the stress concentration factors and damage tensors are known, it is possible to go from one configuration and phase to another. The applied stress is represented by the overall composite stress $\sigma_{i j}$.

\subsection{Stress and strain concentration factors}

In order to move from one phase to another in the network shown in Figure 3.2, it is necessary to determine the stress concentration factor for each configuration.

\subsubsection{Effective configuration}

In the effective configuration all damage has been removed; the material behaves elastically. Therefore, the stress and strain concentration factors are the elastic stress concentration factors 


$$
\begin{aligned}
\bar{\sigma}_{i j}^{m} & =\bar{B}_{i j k l}^{m} \bar{\sigma}_{k l} \\
\bar{\sigma}_{i j}^{f} & =\bar{B}_{i j k l}^{f} \bar{\sigma}_{k l}
\end{aligned}
$$

and

$$
\begin{aligned}
\bar{\varepsilon}_{i j}^{m} & =\bar{A}_{i j k l}^{m} \bar{\varepsilon}_{k l} \\
\bar{\varepsilon}_{i j}^{f} & =\bar{A}_{i j k l k l}^{f} \bar{\varepsilon}_{k l}
\end{aligned}
$$

where $\bar{B}^{m}$ and $\bar{B}^{f}$ are the elastic stress concentration factors and $\bar{A}^{m}$ and $\bar{A}^{f}$ are the elastic strain concentration factors. They can be found by using the Mori-Tanaka method [31] or the Luciano-Barbero method [14]. See Appendix D.

The volume fractions change between configurations because the volume of each phase is altered due to damage. In order to find the volume fractions for the effective configuration, first the uniaxial case is addressed. Let $c^{f}$ and $c^{m}$ the original matrix and fiber volume fractions respectively; $A^{0}$ and $A$ are the original and current area of the composite respectively; $A_{m}$ and $A_{f}$ are the areas of the matrix and fibers respectively. When damage appears in both materials, the amount of area for each phase and the total area are

$$
\begin{aligned}
A^{f} & =c^{f} A^{0}\left(1-\Omega^{f}\right) \\
A^{m} & =c^{m} A^{0}\left(1-\Omega^{m}\right) \\
A & =A^{f}+A^{m}=A^{0}\left[c^{f}\left(1-\Omega^{f}\right)+c^{m}\left(1-\Omega^{m}\right)\right]
\end{aligned}
$$

Therefore, the volume fractions in the effective configuration are

$$
\begin{aligned}
\bar{c}^{f} & =\frac{A^{f}}{A}=\frac{\left(1-\Omega^{f}\right)}{\left(1-\Omega^{f}\right)+\frac{c^{m}}{c^{f}}\left(1-\Omega^{m}\right)} \\
\bar{c}^{m} & =\frac{A^{m}}{A}=\frac{\left(1-\Omega^{m}\right)}{\left(1-\Omega^{m}\right)+\frac{c^{f}}{c^{m}}\left(1-\Omega^{f}\right)}
\end{aligned}
$$

For the three dimensional case and following Voyiadjis and Park [7], the damage variables of Eqn. (3.10) are replaced by equivalent damage tensors defined as 


$$
\Omega_{e q}=\frac{\left(\Omega_{i j} \Omega_{i j}\right)^{1 / 2}}{\Omega^{*}}
$$

where $\Omega^{*}$ is the critical value of the damage variable for the uniaxial case. Therefore the volume fractions in the effective configuration become

$$
\begin{aligned}
\bar{c}^{f} & =\frac{\left(1-\Omega_{e q}^{f}\right)}{\left(1-\Omega_{e q}^{f}\right)+\frac{c^{m}}{c^{f}}\left(1-\Omega_{e q}^{m}\right)} \\
\bar{c}^{m} & =\frac{\left(1-\Omega_{e q}^{m}\right)}{\left(1-\Omega_{e q}^{m}\right)+\frac{c^{f}}{c^{m}}\left(1-\Omega_{e q}^{f}\right)}
\end{aligned}
$$

\subsubsection{Partially damaged configuration}

In the partially damaged configuration, the matrix and fiber are composed of damaged matrix and damaged fibers but no interphase damage. It can be assumed that their volumes do not change with respect to time. Therefore the volume fractions are for the virgin material and constant. But the stress and strain concentration factors vary with time (see Figure 3.2).

Starting with the definitions of stress and strain concentration factors

$$
\begin{aligned}
& \tilde{\sigma}_{i j}^{m}=\tilde{B}_{i j k l}^{m} \tilde{\sigma}_{k l} \\
& \tilde{\sigma}_{i j}^{f}=\tilde{B}_{i j k l}^{f} \tilde{\sigma}_{k l}
\end{aligned}
$$

and

$$
\begin{aligned}
& \tilde{\varepsilon}_{i j}^{m}=\tilde{A}_{i j k l}^{m} \tilde{\varepsilon}_{k l} \\
& \tilde{\varepsilon}_{i j}^{f}=\tilde{A}_{i j k l k l}^{f} \tilde{\varepsilon}_{k l}
\end{aligned}
$$

and considering $(3.13),(3.1)$, and (3.7) yields

$$
\begin{aligned}
\tilde{\sigma}_{i j} & =\tilde{B}_{i j k l}^{-f} M_{k l m n}^{-f} \bar{B}_{m n p q}^{f} \bar{\sigma}_{p q} \\
\tilde{\sigma}_{i j} & =\tilde{B}_{i j k l}^{-m} M_{k l m n}^{-m} \bar{B}_{m n p q}^{m} \bar{\sigma}_{p q}
\end{aligned}
$$


Since the same transformation always maps $\tilde{\sigma}$ to $\bar{\sigma}$

$$
\tilde{B}_{i j k l}^{-f} M_{k l m n}^{-f} \bar{B}_{m n p q}^{f}=\tilde{B}_{i j k l}^{-m} M_{k l m n}^{-m} \bar{B}_{m n p q}^{m}
$$

which eventually leads to

$$
\tilde{B}_{i j k l}^{m}=M_{i j p q}^{-m} \bar{B}_{p q r s}^{m} \bar{B}_{r s m n}^{-f} M_{m n t u}^{f} \tilde{B}_{t u k l}^{f}
$$

and since

$$
\tilde{c}^{m} \tilde{B}_{i j k l}^{m}+\tilde{c}^{f} \tilde{B}_{i j k l}^{f}=I_{i j k l}
$$

a final expression for the stress concentration factor is obtained

$$
\tilde{B}_{i j k l}^{f}=\left(\hat{c}^{m} M_{i j p q}^{-m} \bar{B}_{p q r s}^{m} \bar{B}_{r s m n}^{-f} M_{m n k l}^{f}+\tilde{c}^{f} I_{i j k l}\right)^{-1}
$$

Following the same procedure and using Eqns. (2.18), (3.8), and (3.14) the strain concentration factor for the fiber is found to be

$$
\tilde{A}_{i j k l}^{f}=\left(\tilde{c}^{m} M_{i j p q}^{m} \bar{A}_{p q r s}^{m} \bar{A}_{r s m n}^{-f} M_{m n k l}^{-f}+\tilde{c}^{f} I_{i j k l}\right)^{-1}
$$

\subsubsection{Damaged configuration}

In the damaged configuration, the strains and stresses in the matrix and fiber are the same as in the partially damaged configuration since no additional damage is induced. This means that

$$
\begin{aligned}
& \tilde{\sigma}_{i j}^{m}=\sigma_{i j}^{m} \\
& \tilde{\sigma}_{i j}^{f}=\sigma_{i j}^{f}
\end{aligned}
$$


and

$$
\begin{aligned}
& \tilde{\varepsilon}_{i j}^{m}=\varepsilon_{i j}^{m} \\
& \tilde{\varepsilon}_{i j}^{f}=\varepsilon_{i j}^{f}
\end{aligned}
$$

From the definition of stress and strain concentration factors

$$
\begin{aligned}
\sigma_{i j}^{m} & =B_{i j k l}^{m} \sigma_{k l} \\
\sigma_{i j}^{f} & =B_{i j k l}^{f} \sigma_{k l}
\end{aligned}
$$

and strain concentration factors

$$
\begin{aligned}
\varepsilon_{i j}^{m} & =A_{i j k l}^{m} \varepsilon_{k l} \\
\varepsilon_{i j}^{f} & =A_{i j k l}^{f} \varepsilon_{k l}
\end{aligned}
$$

and by combining Eqns. (3.21), (3.23), and (3.13) the following expressions for the stress concentration factors are derived

$$
\begin{aligned}
B_{i j k l}^{m} & =\tilde{B}_{i j m n}^{m} M_{m n k l}^{d} \\
B_{i j k l}^{f} & =\tilde{B}_{i j m n}^{f} M_{m n k l}^{d}
\end{aligned}
$$

where the superscript $d$ indicates debonding or interphase damage. Similarly, considering Eqns. (3.22), (3.24), and (3.14) the following expressions are found for the strain concentration factors

$$
\begin{aligned}
& A_{i j k l}^{m}=\tilde{A}_{i j m n}^{m} M_{k l m n}^{d} \\
& A_{i j k l}^{f}=\tilde{A}_{i j m n}^{f} M_{k l m n}^{d}
\end{aligned}
$$

\subsection{Stiffness tensors and constitutive model}

To complete the composite damage model, the stiffness tensors and constitutive equations for each phase and configuration are derived here. First, the constitutive equations for the fiber 
and matrix are derived separately. The two constitutive equations are assembled afterwards, producing the partially damaged configuration. To this the debonding damage is added to obtain the final constitutive equation that includes the three types of damage in the damaged configuration.

\subsubsection{Constitutive model for matrix and fiber}

The constitutive equations for the matrix in the effective and partially damaged configurations are

$$
\begin{aligned}
d \bar{\sigma}_{i j}^{m} & =\bar{E}_{i j k l}^{m} d \bar{\varepsilon}_{k l}^{m} \\
d \tilde{\sigma}_{i j}^{m} & =\hat{D}_{i j k l}^{m} d \tilde{\varepsilon}_{k l}^{m}
\end{aligned}
$$

Differentiating Eqns. (3.1) and (3.3)

$$
\begin{aligned}
d \bar{\sigma}_{i j}^{m} & =d M_{i j k l}^{m} \tilde{\sigma}_{k l}^{m}+M_{i j k l}^{m} d \tilde{\sigma}_{k l}^{m} \\
d \bar{\varepsilon}_{i j}^{m} & =d M_{k l i j}^{-m} \tilde{\varepsilon}_{k l}^{m}+M_{k l i j}^{-m} d \tilde{\varepsilon}_{k l}^{m}
\end{aligned}
$$

Furthermore, considering Eqns. (2.43), and (2.68)

$$
\begin{aligned}
d M_{i j k l}^{m} & =\frac{\partial M_{i j k l}^{m}}{\partial \Omega_{m n}^{m}} d \Omega_{m n}^{m}=\frac{\partial M_{i j k l}^{m}}{\partial \Omega_{m n}^{m}} X_{m n p q}^{m} d \tilde{\sigma}_{p q}^{m} \\
d M_{k l i j}^{-m} & =\frac{\partial M_{k l i j}^{-m}}{\partial \Omega_{m n}^{m}} d \Omega_{m n}^{m}=\frac{\partial M_{k l i j}^{-m}}{\partial \Omega_{m n}^{m}} X_{m n p q}^{m} d \tilde{\sigma}_{p q}^{m}
\end{aligned}
$$

By introducing Eqns. (3.28), (3.29), (3.30), and (2.15) into (3.28) the final expression for the damaged stiffness tensor is

$$
\hat{D}_{i j k l}^{m}=N_{i j m n}^{-m} \bar{E}_{m n p q}^{m} M_{k l p q}^{-m}
$$

where 


$$
N_{i j k l}^{m}=\frac{\partial M_{i j p q}^{m}}{\partial \Omega_{m n}^{m}} X_{m n k l}^{m} \tilde{\sigma}_{p q}^{m}+M_{i j k l}^{m}-\bar{E}_{i j r s}^{m} \frac{\partial M_{m n r s}^{-m}}{\partial \Omega_{p q}^{m}} X_{p q k l}^{m} \tilde{E}_{m n t u}^{-m} \tilde{\sigma}_{t u}^{m}
$$

A similar equation can be derived for the fiber, by substituting the index $m$ by $f$.

$$
\hat{D}_{i j k l}^{f}=N_{i j m n}^{-f} \bar{E}_{m n p q}^{f} M_{k l p q}^{-f}
$$

and

$$
N_{i j k l}^{f}=\frac{\partial M_{i j p q}^{f}}{\partial \Omega_{m n}^{f}} X_{m n k l}^{f} \tilde{\sigma}_{p q}^{f}+M_{i j k l}^{f}-\bar{E}_{i j r s}^{f} \frac{\partial M_{m n r s}^{-f}}{\partial \Omega_{p q}^{f}} X_{p q k l}^{f} \tilde{E}_{m n t u}^{-f} \tilde{\sigma}_{t u}^{f}
$$

\subsubsection{Constitutive equation for the composite}

\section{Elastic moduli}

First, the composite elastic modulus in the partially damaged configuration is found. Beginning with the definition of elastic modulus

$$
\tilde{\sigma}_{i j}=\tilde{E}_{i j k l} \tilde{\varepsilon}_{k l}
$$

and combining Eqn. (3.13) and (3.36) results in

$$
\tilde{\sigma}_{i j}^{m}=\tilde{B}_{i j k l}^{m} \tilde{E}_{k l m n} \tilde{\varepsilon}_{m n}
$$

The elastic modulus for the matrix is

$$
\tilde{\sigma}_{i j}^{m}=\tilde{E}_{i j k l}^{m} \tilde{\varepsilon}_{k l}^{m}
$$

Combining Eqns. (3.37), (3.38) and (3.14), one can arrive at 


$$
\tilde{B}_{i j k l}^{m} \tilde{E}_{k l m n}=\tilde{E}_{i j k l}^{m} \tilde{A}_{k l m n}^{m}
$$

A similar equation can be derived for the fiber

$$
\tilde{B}_{i j k l}^{f} \tilde{E}_{k l m n}=\tilde{E}_{i j k l}^{f} \tilde{A}_{k l m n}^{f}
$$

By multiplying the first equation by $\hat{c}^{m}$ and the second by $\tilde{c}^{f}$ and adding them both, taking into account Eqn. (3.18), the final expression for the composite elastic modulus is

$$
\tilde{E}_{i j k l}=\tilde{c}^{m} \tilde{E}_{i j m n}^{m} \tilde{A}_{m n k l}^{m}+\tilde{c}^{f} \tilde{E}_{i j m n}^{f} \tilde{A}_{m n k l}^{f}
$$

A similar approach can be taken to arrive at the composite elastic modulus in the effective and in the damaged configuration.

$$
\begin{aligned}
& \bar{E}_{i j k l}=\bar{c}^{m} \bar{E}_{i j m n}^{m} \bar{A}_{m n k l}^{m}+\bar{c}^{f} \bar{E}_{i j m n}^{f} \bar{A}_{m n k l}^{f} \\
& E_{i j k l}=c^{m} E_{i j m n}^{m} A_{m n k l}^{m}+c^{f} E_{i j m n}^{f} A_{m n k l}^{f}
\end{aligned}
$$

It should be mentioned that in the effective configuration both constituents act elastically. However, since the volume fractions vary, the amount of material is not constant creating a non-linear response for the composite and a variable elastic modulus in Eqn. (3.42).

\section{Stiffness tensor}

The composite stiffness tensor in the effective configuration is found by differentiating Eqn. (3.7) first

$$
d \bar{\sigma}_{i j}^{m}=d \bar{B}_{i j k l}^{m} \bar{\sigma}_{k l}+\bar{B}_{i j k l}^{m} d \bar{\sigma}_{k l}
$$


The stress concentration factor is a function of the volume fraction $\bar{c}^{m}$, therefore Eqn. (3.44) is rewritten as

$$
d \bar{\sigma}_{i j}^{m}=\frac{\partial \bar{B}_{i j k l}^{m}}{\partial \bar{c}^{m}} d \bar{c}^{m} \bar{\sigma}_{k l}+\bar{B}_{i j k l}^{m} d \bar{\sigma}_{k l}
$$

From Eqns. (3.11) and (3.12) the volume fraction $\bar{c}^{m}$ is a function of the matrix and fiber damage

$$
d \bar{\sigma}_{i j}^{m}=\frac{\partial \bar{B}_{i j k l}^{m}}{\partial \bar{c}^{m}}\left(\frac{\partial \bar{c}^{m}}{\partial \Omega_{m n}^{m}} d \Omega_{m n}^{m}+\frac{\partial \bar{c}^{m}}{\partial \Omega_{m n}^{f}} d \Omega_{m n}^{f}\right) \bar{\sigma}_{k l}+\bar{B}_{i j k l}^{m} d \bar{\sigma}_{k l}
$$

and with Eqn. (2.68)

$$
d \bar{\sigma}_{i j}^{m}=\frac{\partial \bar{B}_{i j k l}^{m}}{\partial \bar{c}^{m}}\left(\frac{\partial \bar{c}^{m}}{\partial \Omega_{m n}^{m}} X_{m n p q}^{m} d \tilde{\sigma}_{p q}^{m}+\frac{\partial \bar{c}^{m}}{\partial \Omega_{m n}^{f}} X_{m n p q}^{f} d \tilde{\sigma}_{p q}^{f}\right) \bar{\sigma}_{k l}+\bar{B}_{i j k l}^{m} d \bar{\sigma}_{k l}
$$

Now, differentiating Eqn. (3.1)

$$
d \bar{\sigma}_{i j}^{m}=d M_{i j k l}^{m} \tilde{\sigma}_{k l}^{m}+M_{i j k l}^{m} d \tilde{\sigma}_{k l}^{m}
$$

Introducing Eqn. (3.31) into Eqn. (3.48) and factoring

$$
d \bar{\sigma}_{i j}^{m}=\left(\frac{\partial M_{i j k l}^{m}}{\partial \Omega_{m n}^{m}} X_{m n p q}^{m} \tilde{\sigma}_{k l}^{m}+M_{i j p q}^{m}\right) d \tilde{\sigma}_{p q}^{m}
$$

Combining Eqns. (3.47) and (3.49) and rearranging terms

$$
\begin{aligned}
& \left(\frac{\partial M_{i j k l}^{m}}{\partial \Omega_{m n}^{m}} X_{m n p q}^{m} \tilde{\sigma}_{k l}^{m}+M_{i j p q}^{m}-\frac{\partial \bar{B}_{i j k l}^{m}}{\partial \bar{c}^{m}} \frac{\partial \bar{c}^{m}}{\partial \Omega_{m n}^{m}} X_{m n p q}^{m} \bar{\sigma}_{k l}\right) d \tilde{\sigma}_{p q}^{m} \\
= & \frac{\partial \bar{B}_{i j k l}^{m}}{\partial \bar{c}^{m}} \frac{\partial \bar{c}^{m}}{\partial \Omega_{m n}^{f}} X_{m n p q}^{f} \bar{\sigma}_{k l} d \tilde{\sigma}_{p q}^{f}+\bar{B}_{i j k l}^{m} d \bar{\sigma}_{k l}
\end{aligned}
$$

or 


$$
\begin{aligned}
& \bar{B}_{r s i j}^{-m}\left(\frac{\partial M_{i j k l}^{m}}{\partial \Omega_{m n}^{m}} X_{m n p q}^{m} \tilde{\sigma}_{k l}^{m}+M_{i j p q}^{m}-\frac{\partial \bar{B}_{i j k l}^{m}}{\partial \bar{c}^{m}} \frac{\partial \bar{c}^{m}}{\partial \Omega_{m n}^{m}} X_{m n p q}^{m} \bar{\sigma}_{k l}\right) d \tilde{\sigma}_{p q}^{m} \\
= & \bar{B}_{r s i j}^{-m} \frac{\partial \bar{B}_{i j k l}^{m}}{\partial \bar{c}^{m}} \frac{\partial \bar{c}^{m}}{\partial \Omega_{m n}^{f}} X_{m n p q}^{f} \bar{\sigma}_{k l} d \tilde{\sigma}_{p q}^{f}+d \bar{\sigma}_{r s}
\end{aligned}
$$

Let $\Gamma$ and $\Upsilon$ be defined for the matrix as

$$
\begin{aligned}
\Gamma_{r s p q}^{m} & =\bar{B}_{r s i j}^{-m}\left(\frac{\partial M_{i j k l}^{m}}{\partial \Omega_{m n}^{m}} X_{m n p q}^{m} \tilde{\sigma}_{k l}^{m}+M_{i j p q}^{m}-\frac{\partial \bar{B}_{j j k l}^{m}}{\partial \bar{c}^{m}} \frac{\partial \bar{c}^{m}}{\partial \Omega_{m n}^{m}} X_{m n p q}^{m} \bar{\sigma}_{k l}\right) \\
\Upsilon_{r s p q}^{m} & =\bar{B}_{r s i j}^{-m} \frac{\partial \bar{B}_{i j k l}^{m}}{\partial \bar{c}^{m}} \frac{\partial \bar{c}^{m}}{\partial \Omega_{m n}^{f}} X_{m n p q}^{f} \bar{\sigma}_{k l}
\end{aligned}
$$

Similar expressions are defined for the fibers by interchanging the indexes $m$ and $f$

$$
\begin{aligned}
\Gamma_{r s p q}^{f} & =\bar{B}_{r s i j}^{-f}\left(\frac{\partial M_{i j k l}^{f}}{\partial \Omega_{m n}^{f}} X_{m n p q}^{f} \tilde{\sigma}_{k l}^{f}+M_{i j p q}^{f}-\frac{\partial \bar{B}_{i j k l}^{f}}{\partial \bar{c}^{f}} \frac{\partial \bar{c}^{f}}{\partial \Omega_{m n}^{f}} X_{m n p q}^{f} \bar{\sigma}_{k l}\right) \\
\Upsilon_{r s p q}^{f} & =\bar{B}_{r s i j}^{-f} \frac{\partial \bar{B}_{i j k l}^{f}}{\partial \bar{c}^{f}} \frac{\partial \bar{c}^{f}}{\partial \Omega_{m n}^{m}} X_{m n p q}^{m} \bar{\sigma}_{k l}
\end{aligned}
$$

Eqn. (3.51) is now reduced to

$$
\Gamma_{r s p q}^{m} d \tilde{\sigma}_{p q}^{m}=\Upsilon_{r s p q}^{m} d \tilde{\sigma}_{p q}^{f}+d \bar{\sigma}_{r s}
$$

A similar equation can be found for the fiber

$$
\Gamma_{r s p q}^{f} d \tilde{\sigma}_{p q}^{f}=\Upsilon_{r s p q}^{f} d \tilde{\sigma}_{p q}^{m}+d \bar{\sigma}_{r s}
$$

Following a similar derivation with the strains, the following equations can be found

$$
\begin{aligned}
& \digamma_{r s p q}^{m} d \tilde{\sigma}_{p q}^{m}=\Pi_{r s p q}^{m} d \tilde{\sigma}_{p q}^{f}+d \bar{\varepsilon}_{r s} \\
& \digamma_{r s p q}^{f} d \tilde{\sigma}_{p q}^{f}=\Pi_{r s p q}^{f} d \tilde{\sigma}_{p q}^{m}+d \bar{\varepsilon}_{r s}
\end{aligned}
$$


with

$$
\begin{aligned}
\digamma_{r s p q}^{m} & =\bar{A}_{r s i j}^{-m}\left(\frac{\partial M_{i j k l}^{m}}{\partial \Omega_{m n}^{m}} X_{m n p q}^{m} \tilde{\varepsilon}_{k l}^{m}+M_{k l i j}^{m} \tilde{D}_{k l p q}^{-m}-\frac{\partial \bar{A}_{i j k l}^{m}}{\partial \bar{c}^{m}} \frac{\partial \bar{c}^{m}}{\partial \Omega_{m n}^{m}} X_{m n p q}^{m} \tilde{\varepsilon}_{k l}\right) \\
\Pi_{r s p q}^{m} & =\bar{A}_{r s i j}^{-m} \frac{\partial \bar{A}_{i j k l}^{m}}{\partial \bar{c}^{m}} \frac{\partial \bar{c}^{m}}{\partial \Omega_{m n}^{f}} X_{m n p q}^{f} \tilde{\varepsilon}_{k l} \\
\digamma_{r s p q}^{f} & =\bar{A}_{r s i j}^{-f}\left(\frac{\partial M_{i j k l}^{f}}{\partial \Omega_{m n}^{f}} X_{m n p q}^{f} \tilde{\varepsilon}_{k l}^{f}+M_{k l i j}^{f} \tilde{D}_{k l p q}^{-f}-\frac{\partial \bar{A}_{i j k l}^{f}}{\partial \bar{c}^{f}} \frac{\partial \bar{c}^{f}}{\partial \Omega_{m n}^{f}} X_{m n p q}^{f} \tilde{\varepsilon}_{k l}\right) \\
\Pi_{r s p q}^{f} & =\bar{A}_{r s i j}^{-f} \frac{\partial \bar{A}_{i j k l}^{f}}{\partial \bar{c}^{f}} \frac{\partial \bar{c}^{f}}{\partial \Omega_{m n}^{m}} X_{m n p q}^{m} \tilde{\varepsilon}_{k l}
\end{aligned}
$$

Eliminating $d \bar{\varepsilon}_{r s}$ from Eqns. (3.58) and (3.59)

$$
d \tilde{\sigma}_{i j}^{m}=\left(\Pi_{i j k l}^{f}+\digamma_{i j k l}^{m}\right)^{-1}\left(\Pi_{k l p q}^{m}+\digamma_{k l p q}^{f}\right) d \tilde{\sigma}_{p q}^{f}
$$

Again, from Eqn. (3.58)

$$
d \bar{\varepsilon}_{r s}=\left[\digamma_{r s i j}^{m}\left(\Pi_{i j k l}^{f}+\digamma_{i j k l}^{m}\right)^{-1}\left(\Pi_{k l p q}^{m}+\digamma_{k l p q}^{f}\right)-\Pi_{r s p q}^{m}\right] d \tilde{\sigma}_{p q}^{f}
$$

Finally, combining Eqn. (3.56), (3.65) and considering that $d \bar{\sigma}_{i j}=\bar{D}_{i j k l} d \bar{\varepsilon}_{k l}$

$$
\bar{D}_{r s i j}=\alpha_{r s p q} \beta_{p q i j}^{-1}
$$

with

$$
\begin{aligned}
\alpha_{r s p q} & =\Gamma_{r s p q}^{f}-\Upsilon_{r s i j}^{f}\left(\Pi_{i j k l}^{f}+\digamma_{i j k l}^{m}\right)^{-1}\left(\Pi_{k l p q}^{m}+\digamma_{k l p q}^{f}\right) \\
\beta_{m n p q} & =\digamma_{m n i j}^{m}\left(\Pi_{i j k l}^{f}+\digamma_{i j k l}^{m}\right)^{-1}\left(\Pi_{k l p q}^{m}+\digamma_{k l p q}^{f}\right)-\Pi_{m n p q}^{m}
\end{aligned}
$$

For the composite stiffness tensor in the partially damaged configuration, Eqns. (3.2) and (3.4) are differentiated 


$$
\begin{aligned}
d \tilde{\sigma}_{i j} & =\tilde{c}^{m} d \tilde{\sigma}_{i j}^{m}+\tilde{c}^{f} d \tilde{\sigma}_{i j}^{f} \\
d \tilde{\varepsilon}_{i j} & =\tilde{c}^{m} d \tilde{\varepsilon}_{i j}^{m}+\tilde{c}^{f} d \tilde{\varepsilon}_{i j}^{f}
\end{aligned}
$$

From the definition of stiffness tensor in the partially damaged configuration $d \tilde{\sigma}_{i j}=$ $\tilde{D}_{i j k l} d \tilde{\varepsilon}_{k l}$ and using Eqns. (3.69) and (3.70)

$$
\tilde{c}^{m} d \tilde{\sigma}_{i j}^{m}+\tilde{c}^{f} d \tilde{\sigma}_{i j}^{f}=\tilde{D}_{i j k l}\left(\tilde{c}^{m} d \tilde{\varepsilon}_{k l}^{m}+\tilde{c}^{f} d \tilde{\varepsilon}_{k l}^{f}\right)
$$

and considering the definition of stiffness tensor in the partially damaged configuration for the matrix and fiber

$$
\tilde{c}^{m} d \tilde{\sigma}_{i j}^{m}+\tilde{c}^{f} d \tilde{\sigma}_{i j}^{f}=\tilde{D}_{i j k l}\left(\hat{c}^{m} \tilde{D}_{k l m n}^{-m} d \tilde{\sigma}_{m n}^{m}+\tilde{c}^{f} \tilde{D}_{k l m n}^{-f} d \tilde{\sigma}_{m n}^{f}\right)
$$

Introducing Eqn. (3.64) and rearranging terms

$$
\begin{aligned}
d \tilde{\sigma}_{i j}^{f}= & \left\{\tilde{D}_{i j k l}\left[\frac{\tilde{c}^{m}}{\tilde{c}^{f}} \tilde{D}_{k l m n}^{-m}\left(\Pi_{m n r s}^{f}+\digamma_{m n r s}^{m}\right)^{-1}\left(\Pi_{r s p q}^{m}+\digamma_{r s p q}^{f}\right)+\tilde{D}_{k l p q}^{-f}\right]\right. \\
& \left.-\frac{\tilde{c}^{m}}{\tilde{c}^{f}}\left(\Pi_{i j k l}^{f}+\digamma_{i j k l}^{m}\right)^{-1}\left(\Pi_{k l p q}^{m}+\digamma_{k l p q}^{f}\right)\right\} d \tilde{\sigma}_{p q}^{f}
\end{aligned}
$$

This is possible only if

$$
\begin{aligned}
I_{i j p q}= & \tilde{D}_{i j k l}\left[\frac{\tilde{c}^{m}}{\tilde{c}^{f}} \tilde{D}_{k l m n}^{-m}\left(\Pi_{m n r s}^{f}+\digamma_{m n r s}^{m}\right)^{-1}\left(\Pi_{r s p q}^{m}+\digamma_{r s p q}^{f}\right)+\tilde{D}_{k l p q}^{-f}\right] \\
& -\frac{\tilde{c}^{m}}{\tilde{c}^{f}}\left(\Pi_{i j k l}^{f}+\digamma_{i j k l}^{m}\right)^{-1}\left(\Pi_{k l p q}^{m}+\digamma_{k l p q}^{f}\right)
\end{aligned}
$$

which leads to

$$
\begin{aligned}
\tilde{D}_{i j t u}= & {\left[I_{i j p q}+\frac{\tilde{c}^{m}}{\tilde{c}^{f}}\left(\Pi_{i j k l}^{f}+\digamma_{i j k l}^{m}\right)^{-1}\left(\Pi_{k l p q}^{m}+\digamma_{k l p q}^{f}\right)\right] } \\
& {\left[\frac{\tilde{c}^{m}}{\tilde{c}^{f}} \tilde{D}_{p q m n}^{-m}\left(\Pi_{m n r s}^{f}+\digamma_{m n r s}^{m}\right)^{-1}\left(\Pi_{r s t u}^{m}+\digamma_{r s t u}^{f}\right)+\tilde{D}_{p q t u}^{-f}\right]^{-1} }
\end{aligned}
$$


Finally, following a derivation similar Section 3.9.1 the stiffness tensor in the damaged configuration is computed with

$$
D_{i j k l}=N_{i j m n}^{-d} \tilde{D}_{m n p q} M_{k l p q}^{-d}
$$

\subsection{Summary}

In this chapter the constitutive equations for the matrix, fiber, and composite have been derived. The elastic moduli are necessary for their use in an incremental analysis, since the increments are considered elastic. The stiffness tensors are needed when the material has started damaging. All these tensors are based on the stress and strain concentration factors which have been derived for each configuration. 


\section{Chapter 4}

\section{CONSTANTS DETERMINATION}

\subsection{Introduction}

The uniaxial loading of a bar is the first step in the determination of material properties affecting the damage behavior of the material. In this chapter, procedures to compute these properties will be presented. They are based on the stress-strain curves of the composite and constituents. The determination of material constants for a single material will be described first. Afterwards, procedures involving composite data will be presented. Once found, these properties can be used to analyze a general state of stress problem.

It is assumed that no plastic deformation occurs and all non-linearity in the stress-strain curve is caused exclusively by damage. The damage will be measured by the scalar variable $\Omega$. Many equations used in this chapter are simplifications for the uniaxial case from the general equations developed in Chapters 2 and 3.

\subsection{Thermodynamic force}

For a material subjected to an axial load, the free-energy function $\Psi$ can be written as

$$
\Psi=\frac{1}{2} \frac{\sigma^{2}}{E}
$$

where $\sigma=P / A$ is the applied or Cauchy stress and $E$ is the secant modulus of the stress-strain curve. The secant modulus can be obtained from the stress-strain curve (See 
Figure 4.1) as

$$
E=\frac{\sigma}{\varepsilon}
$$

\section{Strain equivalence principle}

In the strain equivalence principle, the deformations during elastic loading or unloading are the same in the damaged and undamaged configurations, therefore

$$
\varepsilon=\frac{\bar{\sigma}}{\bar{E}}=\frac{\sigma}{E}
$$

where $\sigma=P / A_{\text {eff }}$ is the effective or real stress, taking into account the damage present in the cross sectional area and $\bar{E}$ is the Young's modulus. Considering Eqn. (2.4), the relation between $E$ and the Young's modulus $\bar{E}$ is

$$
E=\bar{E}(1-\Omega)
$$

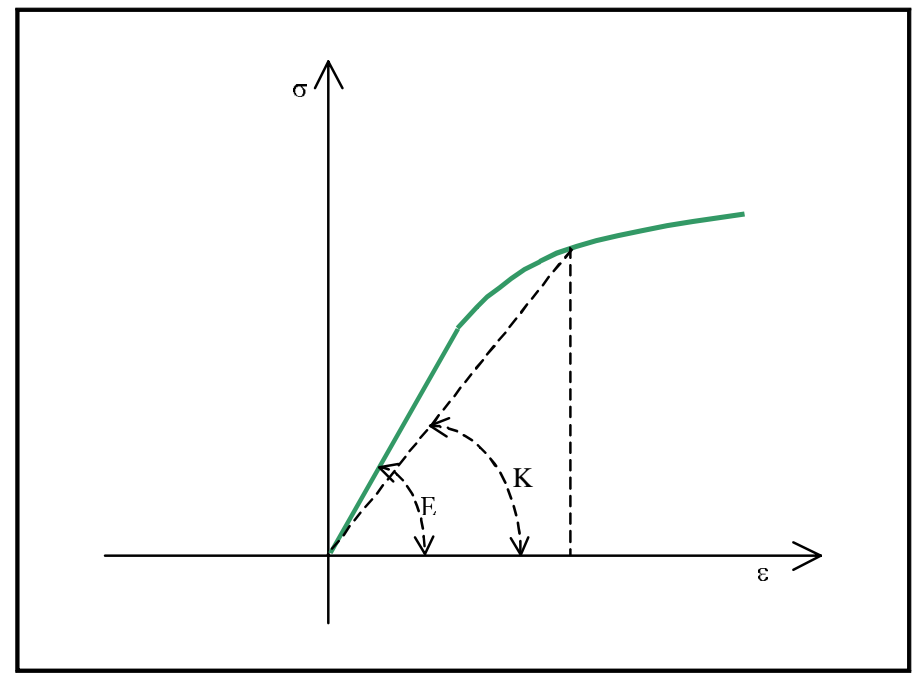

Figure 4.1: Secant modulus $E$

The free energy function is therefore written as 


$$
\Psi=\frac{1}{2} \frac{\sigma^{2}}{\bar{E}(1-\Omega)}
$$

The thermodynamic force conjugate to the damage variable is

$$
Y=-\frac{\partial \Psi}{\partial \Omega}
$$

Using Eqn. (4.5)

$$
Y=-\frac{\sigma^{2}}{2 \bar{E}(1-\Omega)^{2}}
$$

Another useful expression can be obtained by combining Eqns. (4.2), (4.4) and (4.7)

$$
Y=-\frac{\bar{E} \varepsilon^{2}}{2}
$$

At the onset of damage, when $\Omega=0$, the value of $Y$ is

$$
Y_{0}=-\frac{\sigma_{0}^{2}}{2 \bar{E}}
$$

where $\sigma_{0}$ is the stress level when damage starts (damage threshold).

\section{Energy equivalence principle}

In the energy equivalence principle, the energy stored or released during elastic loading or unloading are the same in the damaged and undamaged configurations, therefore

$$
W=\frac{\bar{\sigma}^{2}}{2 \bar{E}}=\frac{\sigma^{2}}{2 E}
$$

And considering again Eqn. (2.4), the relation between $E$ and the original Young's modulus $\bar{E}$ is 


$$
E=\bar{E}(1-\Omega)^{2}
$$

Following a similar approach as the strain equivalence principle, the expressions for the thermodynamic force for the energy equivalence are

$$
\begin{aligned}
Y & =-\frac{\sigma^{2}}{\bar{E}(1-\Omega)^{3}} \\
Y & =-\varepsilon^{2} \bar{E}(1-\Omega) \\
Y_{0} & =-\frac{\sigma_{0}^{2}}{\bar{E}}
\end{aligned}
$$

\subsection{Hardening parameter and damage surface}

Experimental studies have shown that the behavior of damaged materials exhibits hardening. This means that the boundary of the damage surface changes its shape during the loading process. The position of the damage surface depends on a scalar hardening parameter $\kappa$ that monotonically increases as damage propagates. A measure of the hardening parameter can be taken as

$$
\kappa=-\int_{0}^{t} Y \dot{\Omega} d t=-\int_{0}^{\Omega} Y d \Omega
$$

The damage surface can be defined in terms of the hardening tensor $H$ and the thermodynamic force $Y$ as

$$
g=\frac{Y^{2}}{H^{2}}-1
$$

During loading from the damage surface $g=0$, and therefore it is verified that

$$
H=-Y
$$


To complete the model an evolution law for the hardening tensor $H$ is specified. An increment in damage corresponds to an increment in $H$ through the damage multiplier $u$.

$$
d H=u d \Omega
$$

And upon integration

$$
H=u \Omega+H_{0}
$$

The expression $H_{0}$ has been introduced as a integration constant. Therefore, $H_{0}$ is the hardening tensor threshold. The damage multiplier $u$ will vary on the damage state, i.e. $u=u(\kappa)$. A reasonable form for $u$ is

$$
u=u_{0}+\lambda \eta\left(\frac{\kappa}{\lambda}\right)^{\xi}
$$

where $u_{0}$ is the damage multiplier threshold. $\eta$ and $\xi$ are the hardening factor and exponent respectively, materials parameters to be determined. The constant $H_{0}$ is related to the initial location of the damage surface and it is the value of $-Y$ when damage starts appearing. To work with an dimensionless parameter, the following expression is introduced

$$
H_{0}=\lambda v_{0}^{2}
$$

The constant $v_{\mathbf{0}}$ defines the initial threshold against damage, and it can be obtained from the condition that the onset of damage corresponds to the stress level at which the virgin material starts exhibiting nonlinearity. By combining Eqns. (4.19), (4.20), and (4.21) the final expression for the hardening tensor $H$ is

$$
H=\left[u_{0}+\lambda \eta\left(\frac{\kappa}{\lambda}\right)^{\xi}\right] \Omega+\lambda v_{0}^{2}
$$

At the onset of damage $\Omega=0$, and considering Eqn. (4.17) 


$$
H_{0}=-Y_{0}=\frac{\sigma_{0}^{2}}{\bar{E}}
$$

Note that with Eqn. (4.14) $v_{0}$ can be computed as

$$
v_{0}=\frac{\sigma_{0}}{\sqrt{\bar{E} \lambda}}
$$

\subsection{Damage evolution law}

Using the consistency condition $d g=0$, with Eqn. (4.16), the following expression is found

$$
d g=\frac{\partial g}{\partial Y} d Y+\frac{\partial g}{\partial H} d H=0
$$

By differentiating Eqn. (4.16)

$$
d Y-\frac{Y}{H} d H=0
$$

According to Eqn. (4.7), and (4.22), $Y=Y(\sigma, \Omega)$ and $H=H(\kappa, \Omega)$. By differentiating both expressions and taking them into Eqn. (4.26), the following expression is obtained

$$
\frac{\partial Y}{\partial \sigma} d \sigma+\frac{\partial Y}{\partial \Omega} d \Omega-\frac{Y}{H}\left(\frac{\partial H}{\partial \Omega} d \Omega+\frac{\partial H}{\partial \kappa} d \kappa\right)=0
$$

From Eqn. (4.15), it is seen that $d \kappa=\frac{\partial \kappa}{\partial \sigma} d \sigma-Y d \Omega$. Introducing this into Eqn. (4.27), and rearranging terms

$$
\left(\frac{\partial Y}{\partial \sigma}-\frac{Y}{H} \frac{\partial H}{\partial \kappa} \frac{\partial \kappa}{\partial \sigma}\right) d \sigma+\left[\frac{\partial Y}{\partial \Omega}+\frac{Y}{H}\left(Y \frac{\partial H}{\partial \kappa}-\frac{\partial H}{\partial \Omega}\right)\right] d \Omega=0
$$

The damage evolution law is therefore expressed as 


$$
d \Omega=\frac{\frac{Y}{H} \frac{\partial H}{\partial \kappa} \frac{\partial \kappa}{\partial \sigma}-\frac{\partial Y}{\partial \sigma}}{\frac{\partial Y}{\partial \Omega}+\frac{Y}{H}\left(Y \frac{\partial H}{\partial \kappa}-\frac{\partial H}{\partial \Omega}\right)} d \sigma
$$

This equation can also be expressed as

$$
d \Omega=X d \sigma
$$

with

$$
X=\frac{\frac{Y}{H} \frac{\partial H}{\partial \kappa} \frac{\partial \kappa}{\partial \sigma}-\frac{\partial Y}{\partial \sigma}}{\frac{\partial Y}{\partial \Omega}+\frac{Y}{H}\left(Y \frac{\partial H}{\partial \kappa}-\frac{\partial H}{\partial \Omega}\right)}
$$

This is the evolution law for a uniaxial case, based on the general formulation given in Sections $4.2,4.3$, and 4.4 .

\subsection{Graphical determination of damage parameters}

In this section, graphical procedures are presented for the determination of damage parameters needed to describe the uniaxial mode. Due to the initial orthotropy of the threedimensional model, these parameters can be used to model the general case. The procedures are presented for the strain equivalence and energy equivalence approaches.

\subsubsection{Kinematic law $X$}

\section{Strain equivalence principle}

Assuming that $\sigma=\sigma(\varepsilon)$ is known and considering Figure 4.2.

$$
\frac{\sigma}{\varepsilon}=E=\bar{E}(1-\Omega)
$$

or 


$$
\Omega=1-\frac{\sigma}{\bar{E} \varepsilon}
$$

By differentiating Eqn. (4.33) with respect to $\sigma$, the value $X$ of Eqn. (4.30) is obtained

$$
X=\frac{d \Omega}{d \sigma}=-\frac{1}{\bar{E} \varepsilon}\left(1-\frac{\sigma / \varepsilon}{d \sigma / d \varepsilon}\right)=\frac{1}{\bar{E} \varepsilon}\left(\frac{E}{E_{t}}-1\right)
$$

The various terms of Eqn. (4.34) at a point in the stress-strain curve are shown in Figure 4.2. Alternatively, the value of $1 / X$ can be obtained as shown in Figure 4.2, where line $O R$ is parallel to line $Q P$.

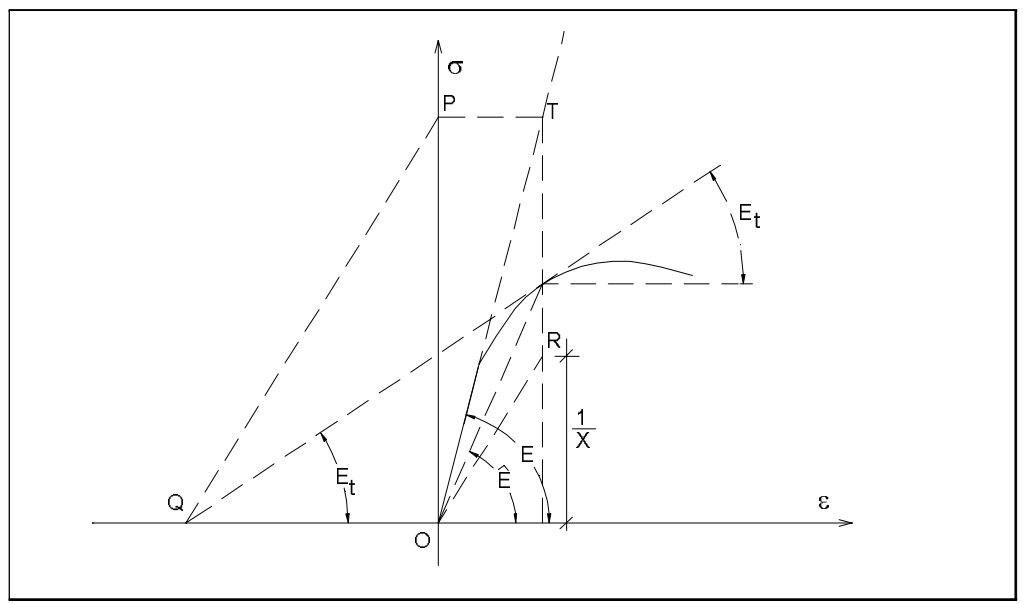

Figure 4.2: Determination of $X$. Strain equivalence principle.

At the onset of damage $\bar{E} \varepsilon=\sigma_{0}$ (damage threshold), $E=\bar{E}$, and Eqn. (4.34) becomes

$$
X_{0}=\frac{1}{\sigma_{0}}\left(\frac{\bar{E}}{E_{t o}}-1\right)
$$

If the material starts damaging when $\varepsilon=0$, the damage threshold $\sigma_{0}=0$. In this case the value of $X$ is indeterminate in Eqn. (4.34) when $\varepsilon=0$. By applying L'Hospital rule and taking the limit, the value of $X_{0}$ becomes

$$
X_{\mathbf{0}}=-\frac{\left.\frac{d^{2} \sigma}{d \varepsilon^{2}}\right|_{\varepsilon=0}}{2 \bar{E} E_{t 0}}
$$


This equation is valid only when the damage threshold is zero.

\section{Energy equivalence principle}

Following a similar approach and taking into consideration Eqn. (4.11), the following expression for $\Omega$ is found

$$
\Omega=1-\sqrt{\frac{\sigma}{\bar{E} \varepsilon}}
$$

and the expression for $X$ is, with the same elements as shown in Figure 4.2

$$
X=\frac{1}{2 \varepsilon \sqrt{\bar{E} E}}\left(\frac{E}{E_{t}}-1\right)
$$

Similarly, a geometrical procedure is explained in Figure 4.3, with the point $T$ defined with the geometric mean of $\bar{E}$ and $E$, or of $\sigma$ and $\bar{\sigma}$, that is

$$
\sigma_{T}=\sqrt{\sigma \bar{\sigma}}
$$

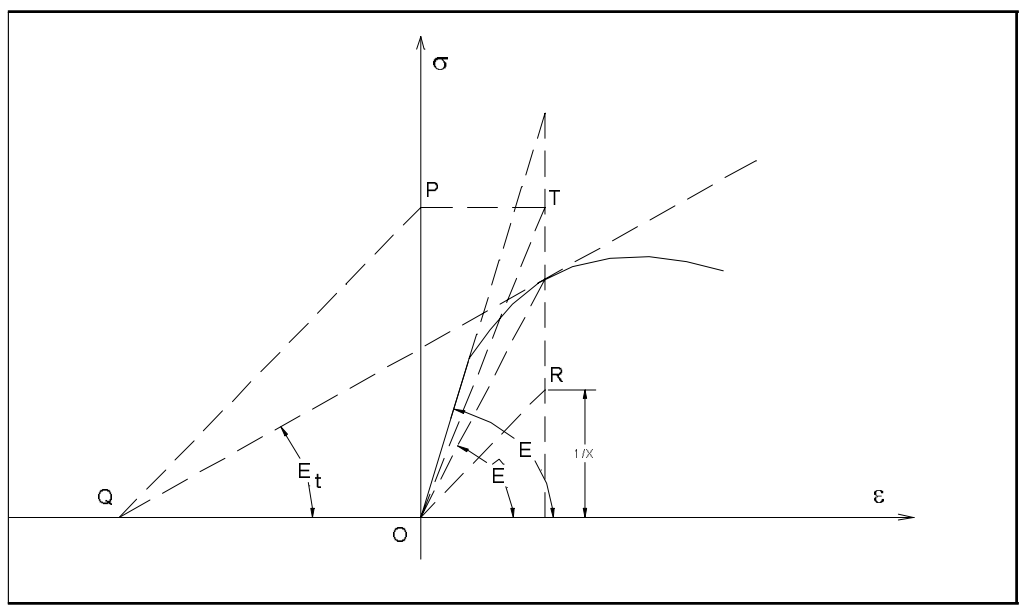

Figure 4.3: Determination of $X$. Energy equivalence principle.

At the onset of damage $\bar{E} \varepsilon=\sigma_{\mathbf{0}}$ (damage threshold), $E=\bar{E}$, and Eqn. (4.38) becomes 


$$
X_{0}=\frac{1}{2 \sigma_{0}}\left(\frac{\bar{E}}{E_{t o}}-1\right)
$$

This value of $X_{\mathbf{0}}$ in the energy equivalence approach is half the value of the strain equivalence principle.

Again, if the damage starts at $\varepsilon=0$ and $\sigma_{0}=0$, Eqn. (4.38) is indeterminate. By applying L'Hospital rule the variation of damage at its onset is

$$
X_{0}=-\frac{\left.\frac{d^{2} \sigma}{d \varepsilon^{2}}\right|_{\varepsilon=0}}{4 \sqrt{\bar{E} E_{t 0}} E_{t 0}}
$$

\subsubsection{Damage multiplier threshold $u_{o}$}

\section{Strain equivalence principle case}

From Eqns. (4.19), (4.23), and (4.12) an expression for the parameter $u$ can be found as

$$
u=\frac{1}{2 \bar{E}}\left[\frac{\sigma^{2}}{(1-\Omega)^{2}}-\sigma_{0}^{2}\right] \frac{1}{\Omega}=\frac{1}{2 \bar{E}}\left[\frac{\sigma^{2}-\sigma_{0}^{2}(1-\Omega)^{2}}{\Omega(1-\Omega)^{2}}\right]
$$

At the onset of damage $\Omega=0, \sigma=\sigma_{0}$, and $u=u_{0}$. Eqn. (4.42) becomes indeterminate. To find the limit when $\Omega=0$, L'Hospital rule is applied again to both parts of the fraction and the following expression is obtained

$$
u_{\mathbf{0}}=\lim _{\Omega \rightarrow 0} u=\frac{1}{\bar{E}}\left[\frac{\sigma \frac{d \sigma}{d \Omega}+\sigma_{\mathbf{0}}^{2}(1-\Omega)}{1+3 \Omega^{2}}\right]_{\Omega=\mathbf{0}}=\frac{1}{\bar{E}}\left[\sigma_{\mathbf{0}}\left(\frac{d \sigma}{d \Omega}\right)_{\Omega=\mathbf{0}}+\sigma_{\mathbf{0}}^{2}\right]
$$

and because of Eqn. (4.30)

$$
u_{0}=\frac{\sigma_{0}}{\bar{E}}\left(\frac{1}{X_{0}}+\sigma_{0}\right)
$$


where $X_{0}$ is the kinematic relation at the start of damage. It is possible to relate $u_{0}$ to geometrical properties of the stress-strain curve. Eqn. (4.35) can be combined with Eqn. (4.44) to yield

$$
u_{0}=\frac{\sigma_{0}^{2}}{\bar{E}-E_{t 0}}=\sigma_{0} \frac{\varepsilon_{0} \varepsilon_{t o}}{\varepsilon^{\prime}}
$$

with the terms $\varepsilon_{0}, \varepsilon_{t 0}$, and $\varepsilon^{\prime}$ explained graphically in Figure 4.4.

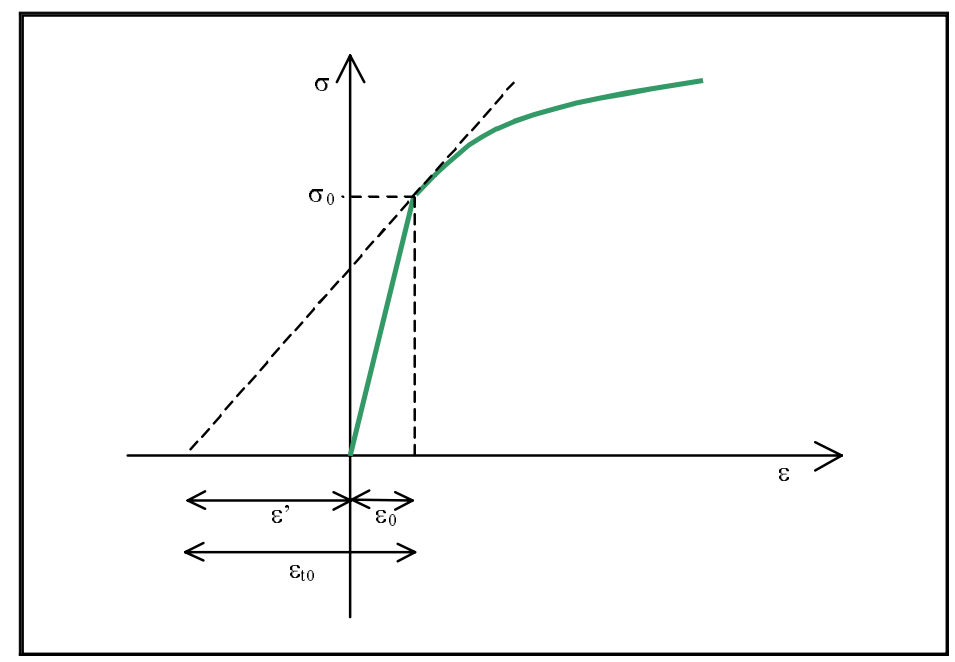

Figure 4.4: Graphical determination of parameter $u_{o}$. Strain equivalence principle.

Some important properties arise when analyzing Eqn. (4.45):

- If the material starts damaging right after loading, meaning $\sigma_{0}=0$, then $u_{0}=0$ as well. Then the curve is non-linear from the origin. The initial Young's modulus is not evident and some other method must be used to find it.

- The value of $u_{0}$ is related to an abrupt change in the $\sigma-\varepsilon$ slope from $\overline{\mathrm{E}}$ to a lesser value $E_{t}$ at the damage onset. If there is no change in slope the value of $u_{0}$ theoretically becomes infinite. But what really happens is that $u_{0}=0$ and the value of $\xi$ is negative yielding an infinite value of $u$ when damage starts.

\section{Energy equivalence principle case}

With the expression for $Y$ for the energy equivalence principle, the equation for $u$ is 


$$
u=\frac{1}{\bar{E}}\left[\frac{\sigma^{2}-\sigma_{0}^{2}(1-\Omega)^{3}}{\Omega(1-\Omega)^{3}}\right]
$$

After applying L'Hospital rule, since the expression is indeterminate at the onset of damage

$$
u_{0}=\frac{\sigma_{0}}{\bar{E}}\left(\frac{2}{X_{0}}+3 \sigma_{0}\right)
$$

and with the value of $X_{0}$ given by Eqn. (4.40)

$$
u_{0}=\frac{\sigma_{0}^{2}}{\bar{E}} \frac{3 \bar{E}+E_{t 0}}{\bar{E}-E_{t 0}}
$$

\subsubsection{Hardening parameter $\kappa$}

By differentiating Eqn. (4.33) with respect to $\varepsilon$

$$
d \Omega=-\frac{1}{\bar{E} \varepsilon}\left(\frac{d \sigma}{d \varepsilon}-\frac{\sigma}{\varepsilon}\right) d \varepsilon
$$

And using the definition of the hardening parameter $\kappa$ given by Eqn. (4.15) with Eqn. (4.8), another expression for the hardening parameter is found

$$
\kappa=\frac{1}{2}\left(\int \sigma d \varepsilon-\int \varepsilon d \sigma\right)=\frac{1}{2}\left(A_{1}-A_{2}\right)
$$

where $A_{1}$ is the area under (horizontal line area in Figure 4.5) and $A_{2}$ is the area left of (vertical line area in Figure 4.5) the $\sigma-\varepsilon$ curve. Furthermore, the hardening parameter $\kappa$ is the area located between the $\sigma-\varepsilon$ curve and the line passing through the point and the origin of coordinates (diagonal line area $A$ in Figure 4.5 ).

The same results are obtained using Eqns. (4.13) and (4.37) instead of Eqns. (4.8) and (4.33) for the energy equivalence principle. 


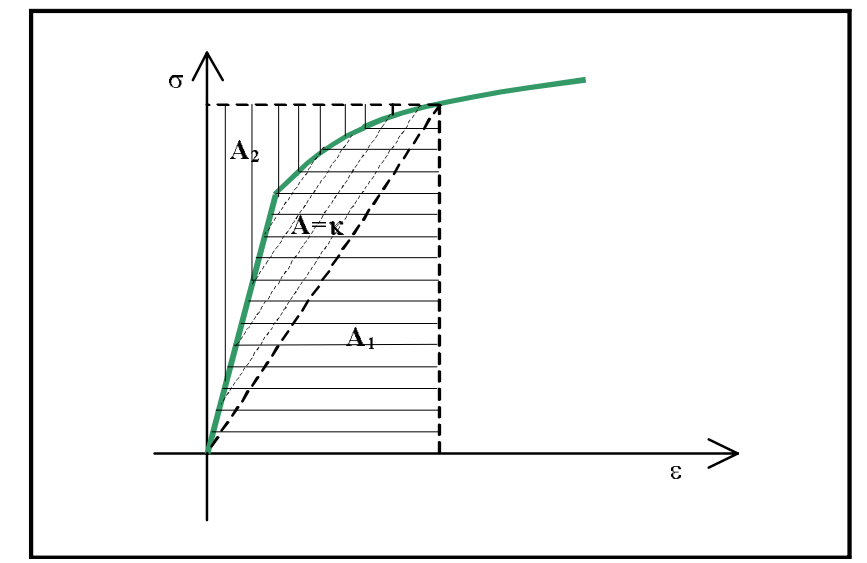

Figure 4.5: Graphical determination of hardening parameter $\kappa$.

Considering the definition of $Y$ for the strain equivalence principle given by 4.7 , the hardening parameter $\kappa$, defined by 4.15 , for this case is

$$
\kappa=\frac{\sigma^{2}}{2 \bar{E}} \int_{0}^{\Omega} \frac{d \Omega}{(1-\Omega)^{2}}=\frac{\sigma^{2}}{2 \bar{E}} \frac{\Omega}{1-\Omega}
$$

while the expression for the energy equivalence principle is

$$
\kappa=\frac{\sigma^{2}}{2 \bar{E}} \frac{\Omega(2-\Omega)}{(1-\Omega)^{2}}
$$

\subsection{Hardening factor $\eta$ and hardening exponent $\xi$}

\subsubsection{Strain equivalence principle case}

From Eqn. (4.22) and using Eqn. (4.21) the following expression can be written

$$
\lambda \eta\left(\frac{\kappa}{\lambda}\right)^{\xi}=\frac{H-H_{0}}{\Omega}-u_{0}
$$

and considering Eqns. (4.17), (4.23), and (4.8) 


$$
\lambda \eta\left(\frac{\kappa}{\lambda}\right)^{\xi}=\frac{\bar{E}}{2} \frac{\varepsilon^{2}-\varepsilon_{0}^{2}}{\Omega}-u_{0}
$$

and using the value of given by Eqn. (4.33)

$$
\lambda \eta\left(\frac{\kappa}{\lambda}\right)^{\xi}=\frac{\bar{E}^{2} \varepsilon}{2} \frac{\varepsilon^{2}-\varepsilon_{0}^{2}}{\bar{E} \varepsilon-\sigma}-u_{0}
$$

By the graphical procedure described in Section 4.5.3, it is possible to find the values $\sigma_{\lambda}$ and $\varepsilon_{\lambda}$ that make $\kappa=\lambda$. Those values in Eqn. (4.55), will allow the determination of $\eta$ as seen below

$$
\eta=\frac{1}{\lambda}\left(\frac{\bar{E}^{2} \varepsilon_{\lambda}}{2} \frac{\varepsilon_{\lambda}^{2}-\varepsilon_{0}^{2}}{\bar{E} \varepsilon_{\lambda}-\sigma_{\lambda}}-u_{0}\right)
$$

Finally, the value of $\xi$ will be determined by plotting the right hand side of Eqn. (4.55), which is usually experimental data, and curve-fitting it with a power law curve whose exponent will be the value of $\xi$ (see Appendix C).

\subsubsection{Energy equivalence principle}

A derivation similar to Eqn. (4.55) yields this equation

$$
\eta\left(\frac{\kappa}{\lambda}\right)^{\xi}=\frac{1}{\lambda}\left\{\frac{\bar{E}\left[-\varepsilon_{0}^{2} \bar{E} \varepsilon+\sqrt{\sigma \bar{E} \varepsilon}\left(\varepsilon^{2}-\varepsilon_{0}^{2}\right)+\sigma \varepsilon^{2}\right]}{\bar{E} \varepsilon-\sigma}-u_{0}\right\}
$$

Finding the values $\sigma_{\lambda}$ and $\varepsilon_{\lambda}$ and that make $\kappa=\lambda, \eta$ can be found as

$$
\eta=\frac{1}{\lambda} \frac{\bar{E}\left[-\varepsilon_{0}^{2} \bar{E} \varepsilon_{\lambda}+\sqrt{\sigma_{\lambda} \bar{E} \varepsilon_{\lambda}}\left(\varepsilon_{\lambda}^{2}-\varepsilon_{0}^{2}\right)+\sigma_{\lambda} \varepsilon_{\lambda}^{2}\right]}{\bar{E} \varepsilon_{\lambda}-\sigma_{\lambda}}-u_{0}
$$

Once the values of $\sigma_{\lambda}$ and $\varepsilon_{\lambda}$ are found, $\eta$ can be determined as 


$$
\eta=\frac{1}{\lambda}\left\{\frac{\bar{E}\left[-\varepsilon_{0}^{2} \bar{E} \varepsilon_{\lambda}+\sqrt{\sigma_{\lambda} \bar{E} \varepsilon_{\lambda}}\left(\varepsilon_{\lambda}^{2}-\varepsilon_{0}^{2}\right)+\sigma_{\lambda} \varepsilon_{\lambda}^{2}\right]}{\bar{E} \varepsilon_{\lambda}-\sigma_{\lambda}}-u_{0}\right\}
$$

And the value of $\xi$ will be determined similarly as in the strain equivalence principle.

\subsection{Stress-strain curve from model}

The nonlinear stress-strain equations can be found in terms of the material properties defined in previous sections.

\subsubsection{Energy equivalence principle}

First Eqn. (4.22) is retrieved and, using Eqns. (4.17), (4.12) and (4.52) written as

$$
\Omega \lambda \eta\left[\frac{\sigma^{2} \Omega(2-\Omega)}{2 \bar{E} \lambda(1-\Omega)^{2}}\right]^{\xi}-\frac{\sigma^{2}}{\bar{E}(1-\Omega)^{3}}+\frac{\sigma_{0}^{2}}{\bar{E}}+\Omega u_{0}=0
$$

Eqn. (4.60) together with Eqn. (4.37) provide the parametric stress-strain curve, $\Omega$ being the parameter.

In the particular case when $\sigma_{0}=0$, Eqn. (4.60) takes the form

$$
\sigma=\left\{\frac{1}{\bar{E} \lambda \eta \Omega(1-\Omega)^{3}}\left[\frac{2 \bar{E} \lambda(1-\Omega)^{2}}{\Omega(2-\Omega)}\right]^{\xi}\right\}^{\frac{1}{2(\xi-1)}}
$$

Alternatively, Eqns. (4.50) and (4.37) can be used instead to provide yet another equation

$$
\left[u_{0}+\lambda \eta\left(\frac{\frac{1}{2}\left(\int \sigma d \varepsilon+\int \varepsilon d \sigma\right)}{\lambda}\right)^{\xi}\right]\left(1-\sqrt{\frac{\sigma}{\bar{E} \varepsilon}}\right)+\lambda v_{0}^{2}-\varepsilon \sqrt{\sigma \bar{E} \varepsilon}=0
$$

An incremental relation can be found by differentiating Eqn. (4.62), and rearranging terms 


$$
\begin{aligned}
& {\left[\eta \xi \varepsilon\left(\frac{\lambda}{\kappa}\right)^{1-\xi}\left(1-\sqrt{\frac{\sigma}{\bar{E} \varepsilon}}\right)+\frac{u_{0}+\lambda \eta\left(\frac{\kappa}{\lambda}\right)^{\xi}}{\sqrt{\sigma \bar{E} \varepsilon}}+\varepsilon \sqrt{\frac{\bar{E} \varepsilon}{\sigma}}\right] d \sigma } \\
= & {\left[\eta \xi \sigma\left(\frac{\lambda}{\kappa}\right)^{1-\xi}\left(1-\sqrt{\frac{\sigma}{\bar{E} \varepsilon}}\right)+\frac{u_{0}+\lambda \eta\left(\frac{\kappa}{\lambda}\right)^{\xi}}{\varepsilon} \sqrt{\frac{\sigma}{\bar{E} \varepsilon}}-3 \sqrt{\sigma \bar{E} \varepsilon}\right] d \varepsilon }
\end{aligned}
$$

This equation can be used to plot the stress-strain curve incrementally.

In terms of the secant and tangent moduli

$$
\begin{aligned}
& {\left[\eta \xi \varepsilon\left(\frac{\lambda}{\kappa}\right)^{1-\xi}\left(1-\sqrt{\frac{E}{\bar{E}}}\right)+\frac{u_{0}+\lambda \eta\left(\frac{\kappa}{\lambda}\right)^{\xi}}{\varepsilon \sqrt{E \bar{E}}}+\varepsilon \sqrt{\frac{\bar{E}}{E}}\right] E_{t} } \\
= & {\left[\eta \xi E \varepsilon\left(\frac{\lambda}{\kappa}\right)^{1-\xi}\left(1-\sqrt{\frac{E}{\bar{E}}}\right)+\frac{u_{0}+\lambda \eta\left(\frac{\kappa}{\lambda}\right)^{\xi}}{\varepsilon} \sqrt{\frac{E}{\bar{E}}}-3 \varepsilon \sqrt{E \bar{E}}\right] }
\end{aligned}
$$

The maximum value of $\sigma$ is given by $d \sigma=0$, which yields the equation

$$
\eta \xi \sigma\left(\frac{\lambda}{\kappa}\right)^{1-\xi}\left(1-\sqrt{\frac{\sigma}{\bar{E} \varepsilon}}\right)+\frac{\left(u_{0}+\lambda \eta\left(\frac{\kappa}{\lambda}\right)^{\xi}\right)}{\varepsilon} \sqrt{\frac{\sigma}{\bar{E} \varepsilon}}-3 \sqrt{\sigma \bar{E} \varepsilon}=0
$$

The solution of Eqn. (4.65) will yield the maximum value $\sigma$ for the model.

\subsection{Application to Janson and Hult model}

In the Janson and Hult model [36] the variation of damage is proportional to the variation of strain.

$$
d \Omega=\frac{\bar{E}}{D} d \varepsilon
$$

where $D$ is called damage parameter. The process of damage starts when $\varepsilon=\varepsilon_{0}$. 


\subsubsection{Strain equivalence principle}

Using Eqn. (4.2) and (4.4), the stress-strain curve when $\varepsilon>\varepsilon_{0}$ is found to be (see Figure 4.6)

$$
\sigma=\bar{E} \varepsilon\left[1-\frac{\bar{E}}{D}\left(\varepsilon-\varepsilon_{0}\right)\right]
$$

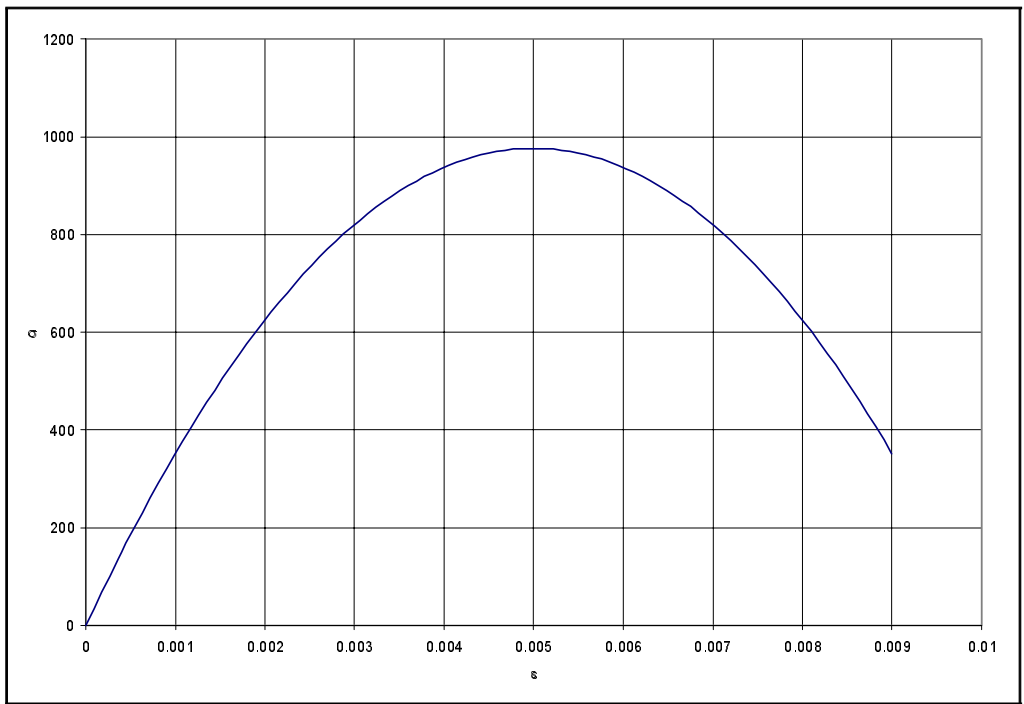

Figure 4.6: Stress-strain curve for a Janson and Hult material.

The tangent modulus at the damage onset is

$$
E_{t o}=\left(\frac{d \sigma}{d \varepsilon}\right)_{\varepsilon=\varepsilon_{0}}=\bar{E}\left[1-\frac{\bar{E}}{D}\left(2 \varepsilon-\varepsilon_{\mathbf{0}}\right)\right]_{\varepsilon=\varepsilon_{0}}=\bar{E}\left(1-\frac{\bar{E}}{D} \varepsilon_{\mathbf{0}}\right)
$$

It can be seen that the original Young's modulus changes abruptly to an always lesser value $E_{t 0}$ shown in Eqn. (4.68).

The area $A$, equal to the hardening parameter $\kappa$, in Figure 4.5 can be found as

$$
\kappa=\frac{\bar{E}^{2}}{6 D}\left(\varepsilon^{3}-\varepsilon_{0}^{3}\right)
$$


The value of $\varepsilon_{\lambda}$ to be used in Eqn. (4.56) is given by

$$
\varepsilon_{\lambda}^{3}=\frac{6 D \lambda}{\bar{E}^{2}}+\varepsilon_{0}^{3}
$$

The value of $u_{0}$ can be found using Eqn. (4.45).

$$
u_{0}=D \varepsilon_{0}
$$

By applying Eqn. (4.56), the value of $\eta$ is obtained as

$$
\eta=\frac{D}{2 \lambda}\left(\varepsilon_{\lambda}-\varepsilon_{0}\right)
$$

\subsubsection{Energy equivalence principle}

Similarly, using Eqn. (4.2) and Eqn. (4.11) the stress strain curve is:

$$
\sigma=\bar{E} \varepsilon\left[1-\frac{\bar{E}}{D}\left(\varepsilon-\varepsilon_{0}\right)\right]^{2}
$$

The maximum stress $\sigma_{\max }$ is computed with

$$
\sigma_{\max }=\frac{4}{27 D^{3}}\left(D+\bar{E} \varepsilon_{0}\right)^{3}
$$

The critical damage $\Omega^{*}$ is found with

$$
\Omega^{*}=\frac{D-2 \bar{E} \varepsilon_{0}}{3 D}
$$

The following expressions are found for $u_{0}$ and $\eta$

$$
u_{0}=\varepsilon_{0}\left(2 D-\sigma_{0}\right)
$$


and

$$
\eta=\frac{\bar{E}\left(\varepsilon_{0}^{2}-\varepsilon_{\lambda}^{2}\right)+\sqrt{D\left[D+\bar{E}\left(\varepsilon_{0}-\varepsilon_{\lambda}\right)\right]}\left(\varepsilon_{0}+\varepsilon_{\lambda}\right)+D\left(-3 \varepsilon_{0}+\varepsilon_{\lambda}\right)}{\lambda}
$$

\subsection{Axially loaded composite bar}

It is assumed that both the matrix and the fibers are elastic. The load and fiber orientations coincide. Only two damage modes are considered: fiber breakage and matrix cracking in the axial direction. If the problem focuses in the axial effects the debonding damage is negligible, even though it may be important for the transverse effects. Also negligible are the transverse damage undergone by the matrix and fiber are negligible.

Two configurations can be can be analyzed for both materials. The damaged configuration (DC) and the effective configuration (EC), obtained by removing all damage in both materials. The relations between the configurations and the different material phases are shown in Figure 4.7 .

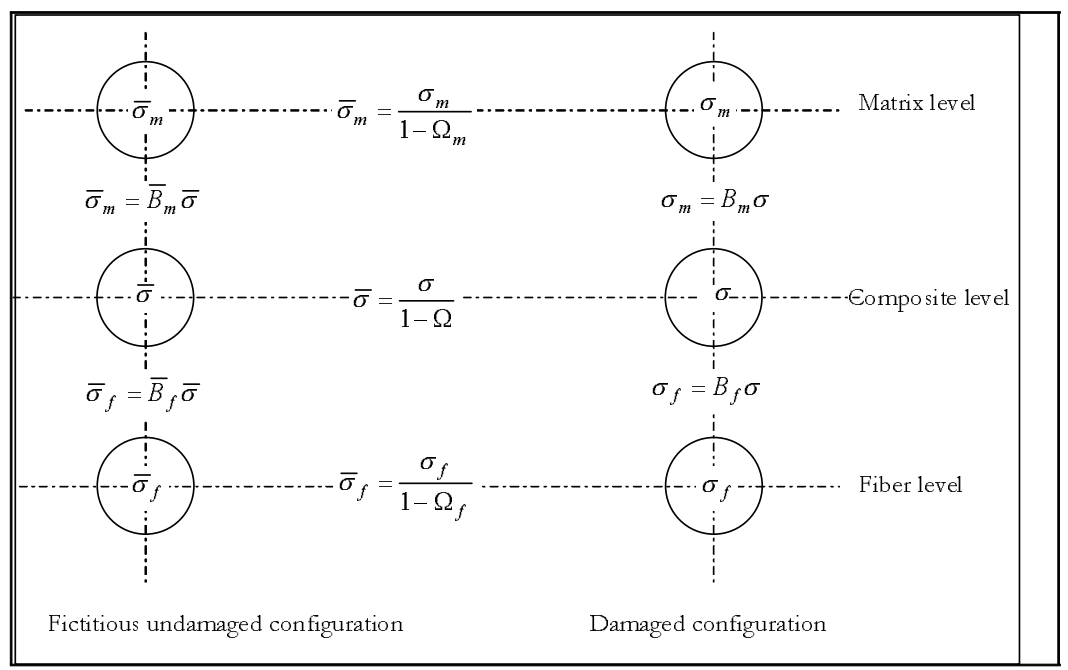

Figure 4.7: Phases and transformation between damage configurations of a composite.

In Figure 4.7, $\bar{B}_{m}$ and $\bar{B}_{f}$ are the matrix and fiber elastic stress concentration factors. By applying the rule of mixtures, good approximations for these factors are 


$$
\begin{aligned}
\bar{B}_{m} & =\frac{\bar{E}_{m}}{\bar{c}_{m} \bar{E}_{m}+\bar{c}_{f} \bar{E}_{f}} \\
\bar{B}_{f} & =\frac{\bar{E}_{f}}{\bar{c}_{m} \bar{E}_{m}+\bar{c}_{f} \bar{E}_{f}}
\end{aligned}
$$

where $\bar{c}_{m}$ and $\bar{c}_{f}$ are the matrix and fiber volume ratios in the $\mathrm{EC}$ and $\overline{\mathrm{E}}_{m}$ and $\overline{\mathrm{E}}_{f}$ are their virgin elastic moduli respectively.

Because of the damage that has appeared the matrix and fiber ratios are not the same as the original ones. The current areas of the matrix, fiber, and composite are

$$
\begin{aligned}
\bar{A}_{m} & =\left(1-\Omega_{m}\right) A_{m}=\left(1-\Omega_{m}\right) c_{m} A_{m} \\
\bar{A}_{f} & =\left(1-\Omega_{f}\right) A_{f}=\left(1-\Omega_{f}\right) c_{f} A_{f} \\
\bar{A} & =\bar{A}_{m}+\bar{A}_{f}=\left[\left(1-\Omega_{m}\right) c_{m}+\left(1-\Omega_{f}\right) c_{f}\right] A
\end{aligned}
$$

Therefore the current volume fractions are

$$
\begin{aligned}
\bar{c}_{m} & =\frac{\bar{A}_{m}}{\bar{A}}=\frac{\left(1-\Omega_{m}\right) c_{m}}{\left(1-\Omega_{m}\right) c_{m}+\left(1-\Omega_{f}\right) c_{f}} \\
\bar{c}_{f} & =\frac{\bar{A}_{f}}{\bar{A}}=\frac{\left(1-\Omega_{f}\right) c_{f}}{\left(1-\Omega_{m}\right) c_{m}+\left(1-\Omega_{f}\right) c_{f}}
\end{aligned}
$$

\subsubsection{Stress concentration factors in the damaged configuration}

To determine the stress concentration factors, $B_{m}$ and $B_{f}$, in the damaged configuration, first the relations between the stresses in the matrix, fiber, and composite in the EC are considered

$$
\begin{aligned}
\bar{\sigma}_{m} & =\bar{B}_{m} \bar{\sigma} \\
\bar{\sigma}_{f} & =\bar{B}_{f} \bar{\sigma}
\end{aligned}
$$

Then, the relations between the stresses in the DC and FUC for the matrix and fiber are 


$$
\begin{aligned}
\bar{\sigma}_{m} & =\frac{\sigma_{m}}{1-\Omega_{m}} \\
\bar{\sigma}_{f} & =\frac{\sigma_{f}}{1-\Omega_{f}}
\end{aligned}
$$

similar to Eqn. (4.81) the relations between the stresses in the matrix, fiber and composite in the DC are

$$
\begin{aligned}
\sigma_{m} & =B_{m} \sigma \\
\sigma_{f} & =B_{f} \sigma
\end{aligned}
$$

By combining Eqns. (4.81), (4.82), and (4.83) the following relation is obtained

$$
\frac{B_{m}}{\left(1-\Omega_{m}\right) \bar{B}_{m}}=\frac{B_{f}}{\left(1-\Omega_{f}\right) \bar{B}_{f}}
$$

Since the stress resultants must be in equilibrium

$$
\sigma_{m} A_{m}+\sigma_{f} A_{f}=\sigma A
$$

It is assumed that the volume fractions in the DC are the same as the original ones. By dividing Eqn. (4.85) by $A$, the following expression is obtained

$$
\sigma_{m} c_{m}+\sigma_{f} c_{f}=\sigma
$$

Combining Eqn. (4.83), Eqn. (4.84), and Eqn. (4.86) the stress concentration factors in the DC are

$$
\begin{aligned}
B_{m} & =\frac{\bar{E}_{m}\left(1-\Omega_{m}\right)}{c_{m} \bar{E}_{m}\left(1-\Omega_{m}\right)+c_{f} \bar{E}_{f}\left(1-\Omega_{f}\right)} \\
B_{f} & =\frac{\bar{E}_{f}\left(1-\Omega_{f}\right)}{c_{m} \bar{E}_{m}\left(1-\Omega_{m}\right)+c_{f} \bar{E}_{f}\left(1-\Omega_{f}\right)}
\end{aligned}
$$


If the strain equivalence concept is used, Eqn. (4.4) can be introduced into Eqn. (4.87) to yield

$$
\begin{aligned}
B_{m} & =\frac{E_{m}}{c_{m} E_{m}+c_{f} E_{f}} \\
B_{f} & =\frac{E_{f}}{c_{m} E_{m}+c_{f} E_{f}}
\end{aligned}
$$

Otherwise, the energy equivalence concept will yield

$$
\begin{aligned}
B_{m} & =\frac{\sqrt{E_{m} \bar{E}_{m}}}{c_{m} \sqrt{E_{m} \bar{E}_{m}}+c_{f} \sqrt{E_{f} \bar{E}_{f}}} \\
B_{f} & =\frac{\sqrt{E_{f} \bar{E}_{f}}}{c_{m} \sqrt{E_{m} \bar{E}_{m}}+c_{f} \sqrt{E_{f} \bar{E}_{f}}}
\end{aligned}
$$

Regardless of the method, it is always verified that

$$
c_{m} B_{m}+c_{f} B_{f}=1
$$

\subsubsection{Determination of in-situ matrix stress-strain curve}

The stress-strain curve for the composite is determined experimentally. A typical curve is shown in Figure 4.9. The stress-strain curve for the fibers can be determined from statistical distribution of fiber strength.

The Weibull expression often used to describe the cumulative probability, $F(\sigma)$, that a fiber of length $\delta$ will fail at stress $\sigma$, is given as

$$
F(\sigma)=1-\exp \left[\frac{-\delta}{L_{0}}\left(\frac{\sigma}{\sigma_{0}}\right)^{m}\right]
$$

The values of $\sigma_{0}$ and $m$, which represents the characteristic strength of the fiber, and the dispersion of the fiber strength, can be determined from fiber strength experiments. $L_{0}$ is the characteristic gauge length corresponding to stress, $\sigma_{0}$. Eqn. (4.91) can be simplified as shown below 


$$
\begin{gathered}
\alpha=\frac{1}{L_{0} \sigma_{0}^{m}} \\
F(\sigma)=1-\exp \left[-\delta \alpha \sigma^{m}\right]
\end{gathered}
$$

If the average strength, $\sigma_{a v}$, for a given gauge length $L_{0}$ is known instead of $\alpha$, Eqn. (4.94) can be used to calculate the value of $\alpha$.

$$
\alpha=\left[\frac{\Gamma\left(1=\frac{1}{m}\right)}{\sigma_{a v}}\right]^{m} \frac{1}{L_{0}}
$$

If no fiber breaks exist initially in the composite, Eqn. (4.93) provides the percentage of fibers in a bundle which are broken as a function of the stress in the unbroken fibers.

In Eqn. (4.93) $\delta$ is the ineffective length which can be obtained analytically for an elastic matrix (Rosen [33]) or numerically for a viscoplastic matrix (Kelly and Barbero [32])

Since $F(\sigma)$ is the amount of fiber damage, it can be used in place of $\Omega$ in Eqn. (4.4) or Eqn. (A.10) to compute the secant modulus $\hat{\sigma}$. Using Eqn. (4.2) the fiber stress-strain curve, in terms of Cauchy stress, is

$$
\sigma_{b}=\bar{E} \exp \left[-\delta \alpha \sigma^{m}\right] \varepsilon
$$

For carbon fiber Toray T300, $\overline{\mathrm{E}}_{f}=230 G p a, \sigma_{a v}=2.63 G p a, m=9$ and fiber diameter $d_{f}=7$ microns. For an elastic matrix the ineffective length

$$
\frac{\delta}{d_{f}}=\frac{1}{2}\left[\left(\frac{1-c_{f}^{1 / 2}}{c_{f}^{1 / 2}}\right)\left(\frac{\bar{E}_{f}}{G_{m}}\right)\right]^{1 / 2} \cosh ^{-1}\left[\frac{1+(1-\phi)^{2}}{2(1-\phi)}\right]
$$

with $\phi=0.9$ (Rosen [33]) and $d_{f}$ the fiber diameter. The stress-strain curve is shown in Figure 4.8 for T300 fibers, $c_{f}=0.6$, in Vinylester Derakane 411-45 (see Table 2.4 in Barbero $[37])$. 


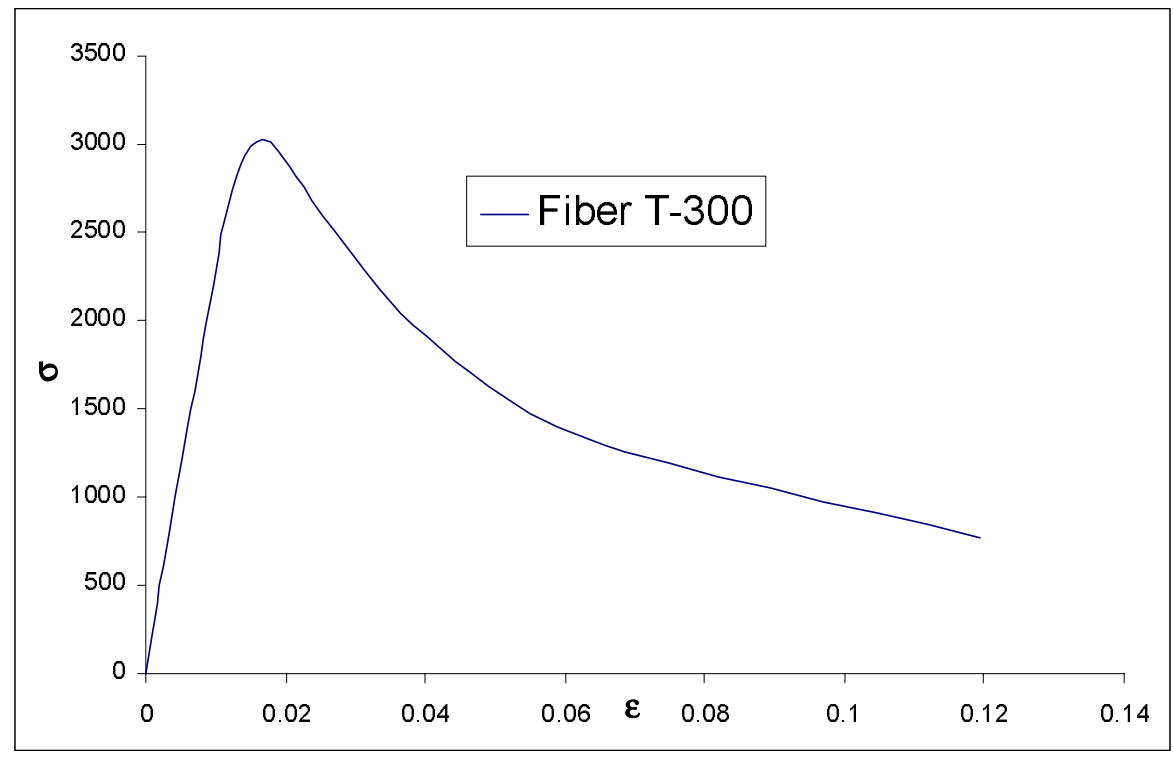

Figure 4.8: Stress strain curve for fiber T300 (Rosen model).

$$
G_{m}=\frac{\bar{E}_{m}}{2\left(1+\nu_{m}\right)}=1.13 \mathrm{Gpa}
$$

Eqn. (4.95) gives the bundle stress (for the bundle of fibers) not the strength in the composite, which is

$$
\sigma_{c}=\sigma_{b}\left[c_{f}+\frac{\bar{E}_{m}}{\bar{E}_{f}}\left(1-c_{f}\right)\right]
$$

according to Eqn. 4.61 in Barbero [37]. The bundle stress is

$$
\sigma_{b}=\sigma(1-\Omega)
$$

and the strain is

$$
\varepsilon=\frac{\sigma_{b}}{E_{f}}=\frac{\sigma_{b}}{\bar{E}_{b}}
$$


Once the stress-strain curve of fiber and composite are known, the stress-strain curve for the matrix is obtained as follows.

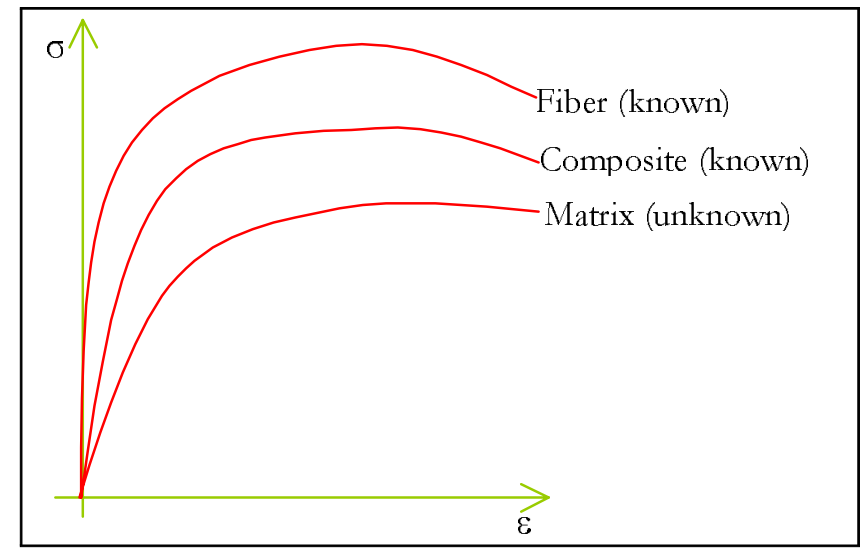

Figure 4.9: Stress-strain curves for a matrix, fiber and the composite.

By combining Eqns. (4.88)b, (4.4), and (4.80) solving the resulting quadratic equation for $1-\Omega_{m}$, the following expression is found

$$
\frac{1-\Omega_{m}}{1-\Omega_{f}}=\frac{\bar{E}_{f}}{2 \bar{E}_{m} B_{f}} \pm \sqrt{\left(\frac{\bar{E}_{f}}{2 \bar{E}_{m} B_{f}}\right)^{2}-\frac{\bar{c}_{f} \bar{E}_{f}}{\bar{c}_{m} \bar{E}_{m}}\left(1-\frac{1}{B_{f}}\right)}
$$

From Eqn. (4.83), the relation between the stresses in the matrix and fiber is

$$
\frac{\sigma_{m}}{\sigma_{f}}=\frac{B_{m}}{B_{f}}=\frac{E_{m}}{E_{f}}=\frac{\bar{E}_{m}\left(1-\Omega_{m}\right)}{\bar{E}_{f}\left(1-\Omega_{f}\right)}
$$

Combining Eqns. (4.100) and (4.101), the final expression for the stress in the matrix is obtained

$$
\sigma_{m}=\frac{\sigma}{2} \pm \sqrt{\left(\frac{\sigma}{2}\right)^{2}-\frac{\bar{c}_{f} \bar{E}_{m}}{\bar{c}_{m} \bar{E}_{f}} \sigma_{f}\left(\sigma_{f}-\sigma\right)}
$$

Eqn. (4.102) can be applied at every point of the composite and fiber stress-strain curves and therefore the matrix curve can be obtained from them. Once this is accomplished, the material parameters for the matrix are obtained according to Sections 4.5 and 4.6. 


\subsubsection{Parameter identification}

As an example of the procedure proposed in Chapter 4, the following set of data will be used to determine all the material constants needed to solve a damage problem.

The data presented in Table 4.1 is known for the matrix and a fiber bundle:

\begin{tabular}{|c|c|c|c|}
\hline \multicolumn{2}{|c|}{ Matrix } & \multicolumn{2}{c|}{ Fiber bundle } \\
\hline$\varepsilon$ & $\sigma$ (Mpa) & $\varepsilon$ & $\sigma$ (Mpa) \\
\hline 0 & 0 & 0 & 0 \\
\hline 0.006875 & 550 & 0.005 & 1460 \\
\hline 0.01 & 680 & 0.01 & 2350 \\
\hline 0.015 & 740 & 0.015 & 2650 \\
\hline 0.02 & 705 & 0.02 & 2750 \\
\hline 0.025 & 525 & 0.025 & 2650 \\
\hline 0.03 & 300 & 0.03 & 2400 \\
\hline 0.035 & 0 & 0.035 & 1800 \\
\hline 0.040 & & 0.04 & 950 \\
\hline 0.045 & & 0.045 & 0 \\
\hline
\end{tabular}

Table 4.1: Experimental data for fiber and composite

A plot of this data is shown in Figure 4.10.

Additional material constants are shown in 4.2 .

\begin{tabular}{|c|c|c|}
\hline & Matrix & Fiber bundle \\
\hline Elastic modulus $\overline{\mathrm{E}}(\mathrm{Mpa})$ & 80000 & 410000 \\
\hline Damage threshold $\sigma_{\mathbf{0}}(\mathrm{Mpa})$ & 550 & 0 \\
\hline Lame constant $\lambda(\mathrm{Mpa})$ & 46154 & 132026 \\
\hline
\end{tabular}

Table 4.2: Additional data for matrix and fiber bundle

The non-linear portion of both curves were fitted with polynomial curves and the slope were computed at the onset of damage. They yielded the results given in Table 4.3 for the tangent modulus $E_{t o}$ at the damage onset. With these values the constants $u_{0}$ can be computed with Eqn. 4.48. The results are also given in Table 4.3.

To determine the constants $\eta$ and $\xi$ Eqn. (4.57) is retrieved and the data fit presented in Appendix $\mathrm{C}$ is used. For that matter the following substitutions are made: 


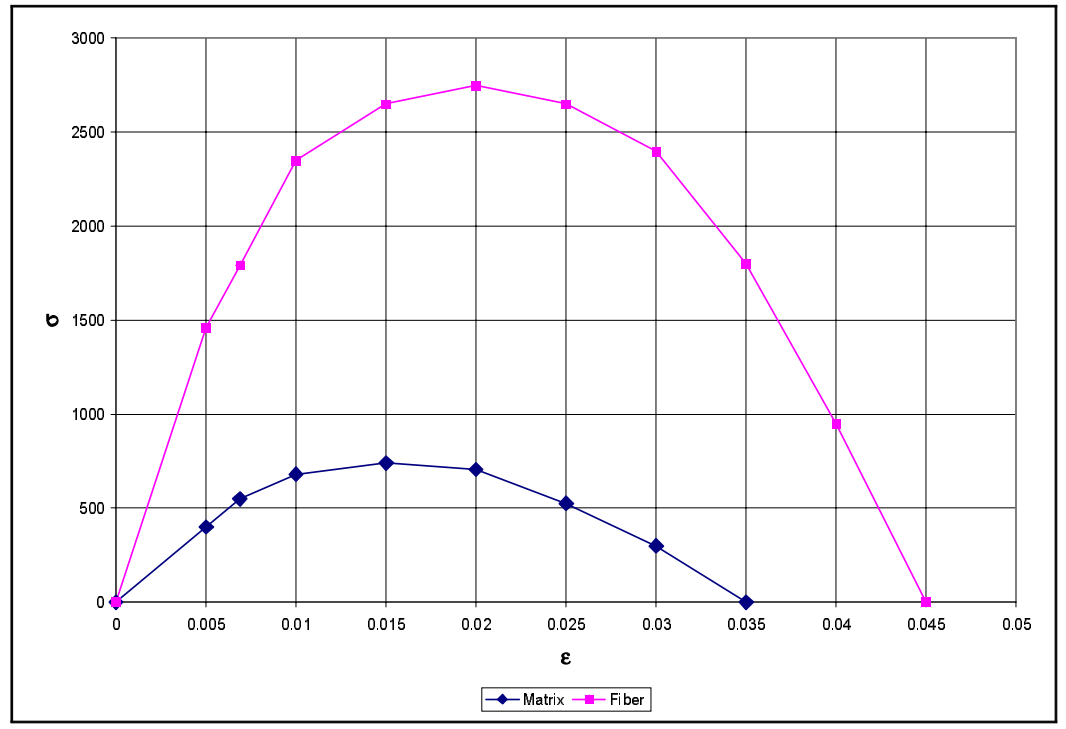

Figure 4.10: Stress-strain curve for matrix and fiber bundle.

\begin{tabular}{|c|c|c|}
\hline & Matrix & Fiber bundle \\
\hline Tanget modulus at damage onset $E_{t o}(\mathrm{Mpa})$ & 54700 & 348000 \\
\hline Hardening parameter $u_{\mathbf{0}}(\mathrm{Mpa})$ & 44.04 & 0 \\
\hline
\end{tabular}

Table 4.3: Tangent modulus at damage onset and hardening parameter

$$
\begin{aligned}
a & =\lambda \eta \\
b & =\xi \\
f(x) & =\frac{\kappa}{\lambda} \\
y & =\frac{\bar{E}\left[-\varepsilon_{0}^{2} \bar{E} \varepsilon+\sqrt{\sigma \bar{E} \varepsilon}\left(\varepsilon^{2}-\varepsilon_{0}^{2}\right)+\sigma \varepsilon^{2}\right]}{\bar{E} \varepsilon-\sigma}-u_{0}
\end{aligned}
$$

Applying the equations presented in Appendix $\mathrm{C}$ with the known points of the stressstrain curves the values in Table 4.4 are found:

\subsubsection{Accuracy of the model}

In order to measure the approximation of the model given, a uniaxial damage model will be solved. First, the experimental data will be used to solve and then the model presented. 


\begin{tabular}{|c|c|c|}
\hline & Matrix & Fiber bundle \\
\hline$a$ & 26.80 & 94.66 \\
\hline$b=\xi$ & -0.8013 & 0.2489 \\
\hline$\eta$ & $1.0658 \mathrm{E}-7$ & 0.01349 \\
\hline
\end{tabular}

Table 4.4: Values of $\eta$ and $\xi$

Using Eqn. (4.85) the composite stress-strain curve can be determined as shown in Figure 4.11 .

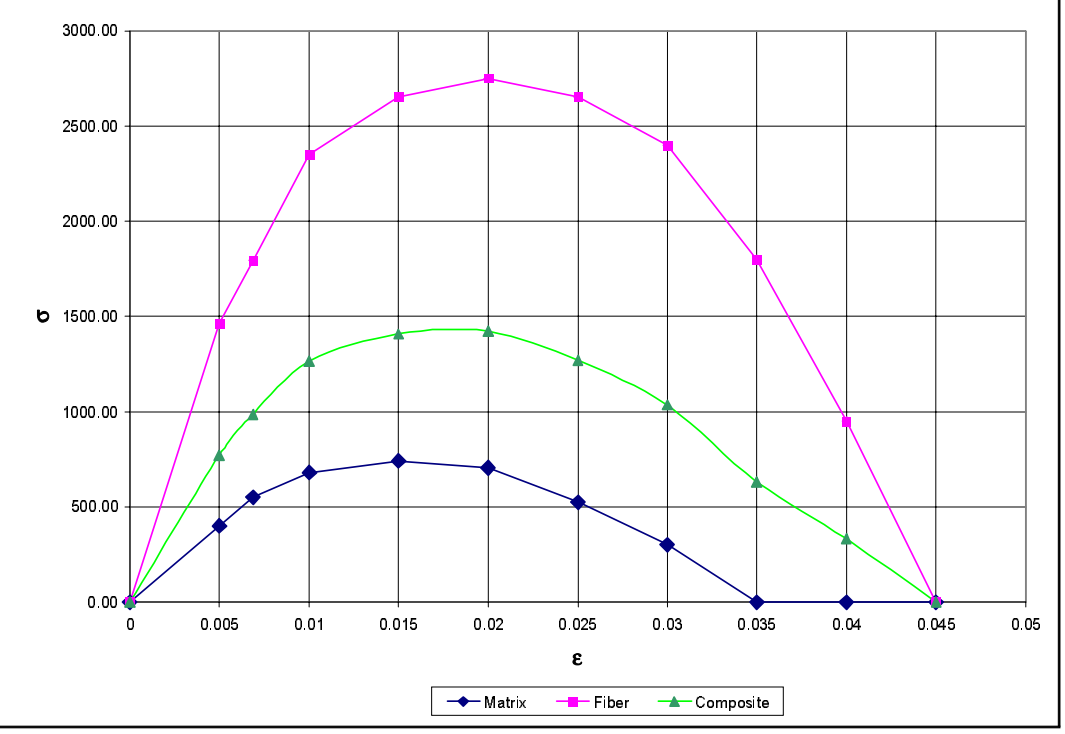

Figure 4.11: Composite curve obtained from matrix and fiber curve.

For the purpose of comparison, Figure shows the fiber stress-strain curve obtained with the model parameters and the real stress-strain curve.

\subsection{Transversely loaded composite bar}

In this case the direction of the uniaxial load is perpendicular to the principal direction of the material (the direction of the fibers, see Figure 4.13). It is assumed that the two damage modes are matrix cracking and fiber and matrix debonding. No fiber breakage is expected to occur. 


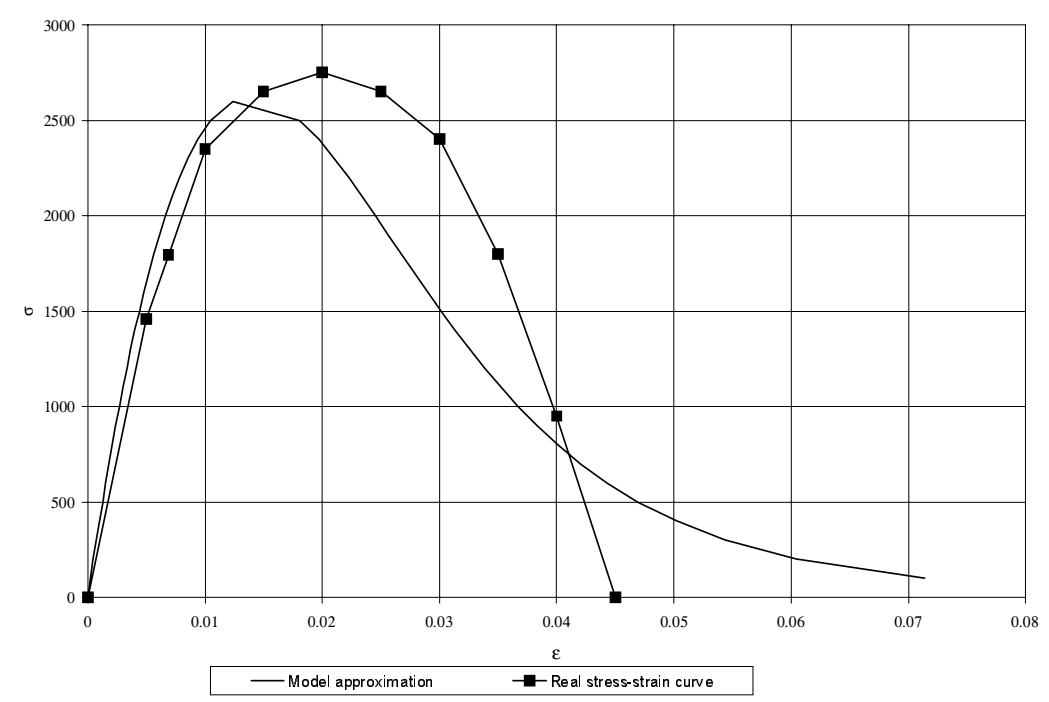

Figure 4.12: Comparison of model and real stress-strain curve for the fibers.

Debonding damage is measured by a scalar $\Omega_{d}$ defined as the length of the fibers that are not in contact with the matrix (Figure 4.14)

$$
\Omega_{d}=\frac{\sum l_{d}}{\sum l_{0}}
$$

For a transversely loaded bar, the stresses in the matrix, fiber and composite are the same in any configuration. However, the strains are different for each phase. The rule of mixtures provides a good approximation for the relation between strains

$$
\varepsilon^{m} c^{m}+\varepsilon^{f} c^{f}=\varepsilon
$$

where:

$\varepsilon^{m}=$ strain in the matrix

$\varepsilon^{f}=$ strain in the fiber

$\varepsilon=$ strain in the composite 


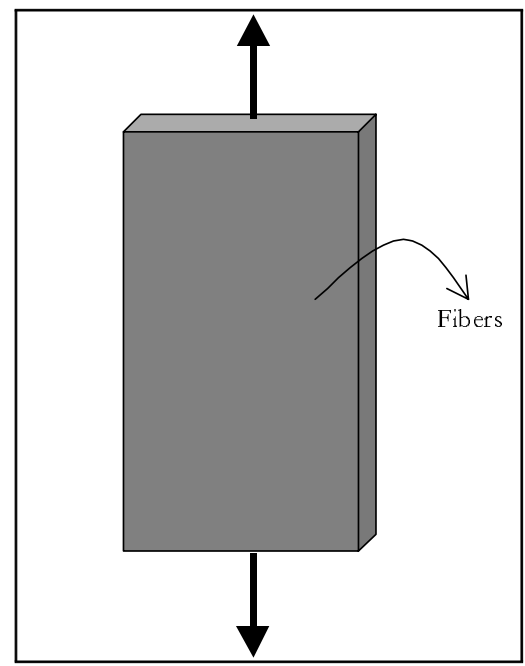

Figure 4.13: Bar transversely loaded.

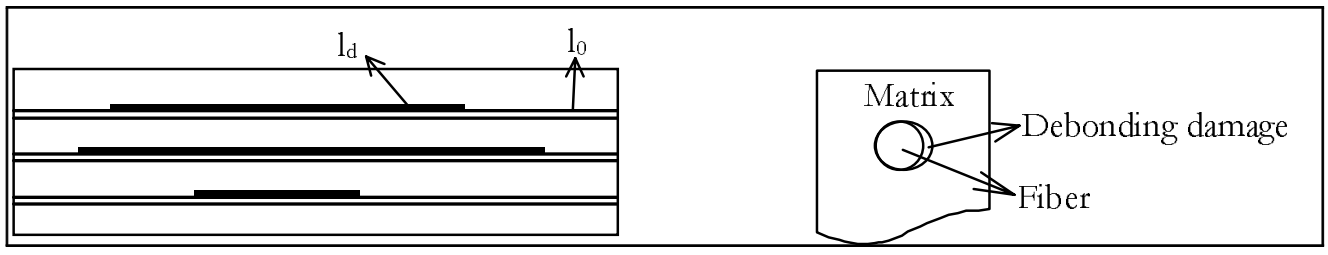

Figure 4.14: Quantification of debonding damage.

Since the stresses are the same in each phase

$$
\begin{aligned}
\sigma^{m} & =\sigma^{f}=\sigma^{c} \\
E^{m} \varepsilon^{m} & =E^{f} \varepsilon^{f}=E^{c} \varepsilon^{c}
\end{aligned}
$$

Combining Eqns. (4.104) and (4.105), a relation between the secant moduli is obtained

$$
\frac{c^{m}}{E^{m}}+\frac{c^{f}}{E^{f}}=\frac{1}{E}
$$

The absence of fiber damage allows a serial transformation between the stresses in the damaged and effective configurations, as shown in Figure 4.15. The equations governing this transformation are 


$$
\begin{aligned}
\bar{\sigma} & =\frac{\tilde{\sigma}}{1-\Omega^{m}} \\
\tilde{\sigma} & =\frac{\sigma}{1-\Omega^{d}}
\end{aligned}
$$

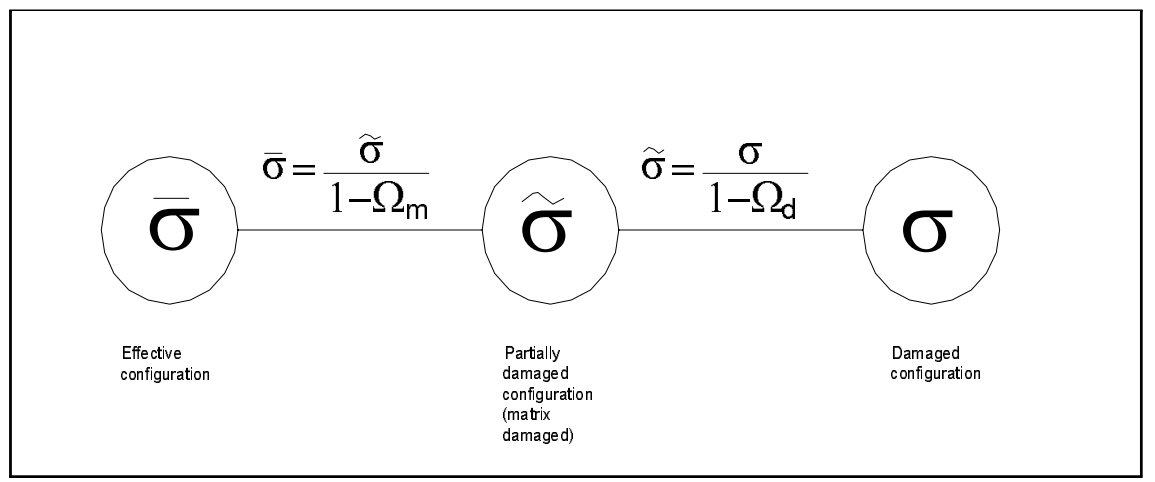

Figure 4.15: Stress transformations for a transversely loaded bar.

What is usually known are the stress-strain curve of the composite and of the matrix, the latter obtained through a longitudinal uniaxial test. What is usually required to complete the debonding damage model is the stress-strain curve of the composite due exclusively by interphase damage with no matrix damage. From this curve the debonding damage parameters will be determined. The known composite stress-strain curve contains the matrix damage, which must be subtracted.

The stress-strain curve of the composite is $\sigma=f_{1}(\varepsilon)$, the stress-strain curve of the matrix is $\hat{\sigma}^{m}=\hat{\sigma}=f_{2}\left(\hat{\varepsilon}^{m}\right)$. Substituting in Eqn. (4.108)

$$
f_{2}\left(\hat{\varepsilon}^{m}\right)=\frac{f_{1}\left(\varepsilon^{c}\right)}{1-\Omega^{d}}
$$

The energy equivalence principle applied to the PDC and DC yields

$$
\hat{\varepsilon}^{m}=\frac{\tilde{E}}{\tilde{E}^{m}} \tilde{\varepsilon}=\frac{\tilde{E}}{\tilde{E}^{m}}\left(1-\Omega^{d}\right) \varepsilon
$$

The relation between strains in the damaged and partially damaged configurations, given by the energy equivalence principle, is 


$$
\tilde{\varepsilon}=\left(1-\Omega^{d}\right) \varepsilon
$$

Eqn. (4.111) is introduced into Eqn. (4.110) to yield

$$
\hat{\sigma}\left[\frac{\tilde{E}}{\tilde{E}^{m}}\left(1-\Omega^{d}\right) \varepsilon^{c}\right]=\frac{\sigma\left(\varepsilon^{c}\right)}{1-\Omega^{d}}
$$

From Eqn. (4.106)

$$
E=\frac{\tilde{E}^{m} \tilde{E}^{f}}{\tilde{c}^{m} \tilde{E}^{f}+\tilde{c}^{f} \tilde{E}^{m}}
$$

From Eqn. (4.11)

$$
\begin{aligned}
\tilde{E}^{m} & =\bar{E}^{m}\left(1-\Omega^{m}\right)^{2} \\
\tilde{E}^{f} & =\bar{E}^{f}
\end{aligned}
$$

It is also assumed that the volume fractions in the damaged and partially damaged configurations are the same

$$
\begin{aligned}
\tilde{c}^{m} & =c^{m} \\
\tilde{c}^{f} & =c^{f}
\end{aligned}
$$

Introducing Eqns. (4.113), (4.114), and (4.115) into Eqn. (4.112)

$$
\hat{\sigma}\left[\frac{\bar{E}^{f}}{c^{m} \bar{E}^{f}+c^{f} \bar{E}^{m}\left(1-\Omega^{m}\right)^{2}}\left(1-\Omega^{d}\right) \varepsilon^{c}\right]=\frac{\sigma\left(\varepsilon^{c}\right)}{1-\Omega^{d}}
$$

Eqn. (4.116) allows the determination of $\Omega^{d}$ for every value of $\varepsilon^{c}$. Let $\sigma^{\prime}$ be the stress in the composite due exclusively to debonding damage. Introducing Eqn. (4.37) in Eqn. (4.116) 


$$
\hat{\sigma}\left[\frac{\bar{E}^{f}}{c^{m} \bar{E}^{f}+c^{f} \bar{E}^{m}\left(1-\Omega^{m}\right)^{2}} \sqrt{\frac{\sigma^{\prime}}{\bar{E} \varepsilon} \varepsilon}\right]=\frac{\sigma(\varepsilon)}{\sqrt{\frac{\sigma^{\prime}}{E \varepsilon}}}
$$

where the elastic composite modulus to be used in Eqn. (4.117) is

$$
\bar{E}=\frac{\bar{E}^{m} \bar{E}^{f}}{c^{m} \bar{E}^{m}+c^{f} \bar{E}^{f}}
$$

\subsection{Summary}

As a summary of the variables introduced in this chapter, Table 4.5 describes the parameters used in the model and the equation number where they can be found for both approaches:

\begin{tabular}{|c|c|c|c|}
\hline Parameter & Meaning & Strain equivalence approach & Energy equivalence approach \\
\hline$\Psi$ & free energy function & Eqn. 4.5 & \\
\hline$E$ & secant elastic modulus & Eqn. 4.4 & Eqn. 4.11 \\
\hline$Y$ & thermodynamic force & Eqn. 4.7 & Eqn. 4.12 \\
\hline$Y_{0}$ & thermodynamic force at damage onset & Eqn. 4.9 & Eqn. 4.13 \\
\hline$\kappa$ & scalar hardening parameter & Eqn. 4.51 & Eqn. 4.52 \\
\hline$H$ & tensorial hardening parameter & Eqn. 4.22 & Eqn. 4.22 \\
\hline$u$ & hardening parameter & Eqn. 4.42 & Eqn. 4.46 \\
\hline$u_{0}$ & hardening parameter at damage onset & Eqn. 4.44 & Eqn. 4.47 \\
\hline$\eta$ & shape of model stress-strain curve & Eqn. 4.56 & Eqn.4.59 \\
\hline$\xi$ & size of model stress-strain curve & Data fit & Data fit \\
\hline$X$ & damage evolution law & Eqn. 4.34 & Eqn. 4.38 \\
\hline$E_{t}$ & tangent modulus & curve & curve \\
\hline$X_{0}$ & damage evolution law at damage onset & Eqn. 4.35 & Eqn. 4.40 \\
\hline \multirow[t]{2}{*}{$\bar{B}$} & \multirow{2}{*}{$\begin{array}{l}\text { stress concentration factor } \\
\text { in effective configuration }\end{array}$} & Eqn. 4.78 & Eqn. Eqn. 4.78 \\
\hline & & & \\
\hline$\hat{B}$ & stress concentration factor in damaged configuration & Eqn. 4.88 & Eqn. 4.89 \\
\hline
\end{tabular}
strain equivalence and energy equivalence.

Table 4.5: Summary of material parameters 


\section{Chapter 5}

\section{FINITE ELEMENT IMPLEMENTATION}

\subsection{Introduction}

The use of the finite element method as a tool for the solution of composite micromechanics problems has become increasingly popular in recent years because of several important factors. First, the adaptation of a finite element formulation for solution utilizing a high-speed digital computer is straightforward and not problem-specific. Second, the types of approximations which are present to some degree in all of the analytical models are not inherent to the finite element method.

A large number of nonlinear finite element micromechanics solutions have been obtained for many technically relevant composite material problems. Among the more notable papers found in the literature are the applications of the periodic hexagonal array model of Teply and Dvorak [22], the studies of whisker-reinforced aluminum by Christman and coworkers [23], and the analysis of the response of viscoplastic matrix composite under thermal and mechanical loads by Sherwood and Boyle [24].

The model presented in this dissertation has been implemented into a finite element program, since this has proved to be the best tool for validating this type of model. The main features of the program are:

- A plane stress or plane strain element with 4,8 , or 9 nodes.

- A modified Newton-Raphson method is used, updating the stiffness tensor at the be- 
ginning of each increment.

- Dynamic storage is used, using RVAR array for real values and IVAR array for integer values. Pointers are defined for each vector or matrix used in the program.

Only the finite element implementation aspects are described in this chapter with reference to the theoretical equations in previous chapters. For details regarding standard finite element method procedures, please refer to Chrisfield [34] and Owen and Hinton [29].

\subsection{Damaged stiffness and evolution law}

The two most important questions in any damage model are the computation of the stiffness tensor and the damage evolution law. These are performed through the subroutines DMAT and DAMAGE. Differently from the plasticity case, the damage surface is represented by Eqn. (2.50) in the thermodynamic force space $Y_{i j}$ instead of the stress space.

\subsection{Nodal forces}

Nodal forces are produced by the stress field that satisfies the elasto-damage conditions. The difference between these forces and the applied load gives the residual forces.

In the most general situation, an element is inside the damage surface as shown in Figure 5.1. During the application of an increment of load, that element (or part of it) may damage. All stress and strains quantities are monitored at each Gaussian integration point and therefore whether damage has occurred can be determined at such points. Consequently an element can have partially-elastic deformation and partially-damaged deformation. For any load increment it is necessary to determine what proportion is elastic $(k-1)$ and which part (k) produces damage. Then adjust the stress and strain terms until the yield criterion and the constitutive laws are satisfied. The procedure adopted, based on Owen and Hinton [29], is as follows:

1. The applied loads for the $i^{\text {th }}$ iteration are the residual forces $R^{i-1}$ which produce displacement increments $d \delta^{i}$ and strain increments $d \varepsilon^{i}$.

2. The incremental stress changes $d \sigma_{e}^{i}$ are computed assuming elastic behavior 


$$
d \sigma_{i j(e)}=E_{i j k l} d \varepsilon_{k l}
$$

where the subscript $e$ stands for elastic stress.

3. Accumulate the total stress for each element Gauss point as $\sigma_{e}^{i}=\sigma^{i-1}+d \sigma_{e}^{i}$ where $\sigma^{i-1}$ are the converged stresses for the iteration $i-1$.

4. The next step depends on whether or not damage took place at the Gauss point during: the $i-1$ iteration. The presence of damage is revealed by the value of $g$ reached at the $i-1$ iteration, which was computed with Eqn. (2.50) and stored in memory. If $g^{i-1}=0$, that means the point was in the damage surface. If $g^{i-1}<0$, it means the point was inside the damage surface; that is no damage occurred.

5. If the Gauss point was in the damage surface, it is now necessary to determine whether the point is loading or unloading. To do so, one can compute the value of $g$ in the current iteration. If the value of $g<0$, it means that the point is unloading elastically and therefore the stress increment $d \sigma_{e}^{i}$ computed is the real one. In this case $k=0$ since the behavior is all elastic. If $g=0$ it means that the loading is neutral. If $g>0$ it means that the point is still loading. The $g$ computed at this point is not the real one, since in its computation the damage Eqn. (2.50) is used and it has not been updated. Instead, the old damage variables have to be used. It is possible to know whether $g>0$ even though it is not possible to know its real value at this time. For this case, the increment in damage is found with the variable $X_{i j k l}$ of Eqn. (4.23) using the value of $\sigma^{i-1}$. With the value of $X_{i j k l}$, the tangent stiffness tensor $D_{i j k l}$ is computed with Eqn. (2.79). Then, the real increment in stress is found with $d \sigma_{i j}=D_{i j k l} d \varepsilon_{k l}$. Now, the increment in stress is found with Eqn. (4.22), and the final state of stress is computed. Obviously, for this case $k=1$, since there is no elastic behavior.

6. If the Gauss point was inside the damage surface, again it is necessary to compute the current $g$ value. If $g<0$, the Gauss point is still elastic and therefore the elastic increment is the real one. If $g>0$, it means that part of the behavior is elastic and part is damaged. Then, it is necessary to find the elastic $(k-1)$ and damage part $(k)$. To do so, first the value of the stress state that will reach just the damage surface is computed. Such value can be defined as a factor of $\sigma^{i-1}$, like $n \sigma^{i-1}$. At this point, it is helpful to remember that the load path up to the damage surface is in the straight line 
connecting the position of the point in the $Y$ space and the origin of coordinates. This is a characteristic of the damage phenomenon whereby the elastic unloading produces no residual deformation, and therefore the unloading and the elastic loading must be in the path described above. Also, it should be remembered that according to the definition given by Eqn. (2.48), $Y$ is function of the square of $\sigma$, that is $Y=f\left(\sigma^{2}\right)$.

With that explanation, the following equations can be written

$$
\begin{aligned}
g^{i-1} & =H_{i j} H_{j k} Y_{k l} Y_{l i}-1 \\
0 & =n^{4} H_{i j} H_{j k} Y_{k l} Y_{l i}-1
\end{aligned}
$$

From these two equations

$$
n=\left(\frac{1}{g^{i-1}+1}\right)^{1 / 4}
$$

The value $n \sigma^{i-1}$ is used to find the value of $X$ just at the damage surface. Actually, Eqn. (2.69) which gives the expression for $X$ can be used directly by introducing the factor $n$ appropriately in selected terms, and use $\sigma^{i-1}$ in the computation of $X$. For instance, $\frac{\partial g}{\partial Y_{m n}}$ is function of $n^{2}, \frac{\partial Y_{m n}}{\partial \sigma_{k l}}$ is function of $n, Y$ is a function of $n^{2}, \frac{\partial Y_{m n}}{\partial \Omega_{p q}}$ is function of $n^{2}, \frac{\partial g}{\partial H_{m n}}$ is function of $n^{4}$. With this, the expression for $X$ is given below

$$
X_{i j k l}=-\frac{\frac{\partial g}{\partial Y_{m n}} \frac{\partial Y_{m n}}{\partial \sigma_{k l}} \frac{\partial g}{\partial Y_{i j}}}{n\left[\frac{\partial g}{\partial Y_{m n}} \frac{\partial Y_{m n}}{\partial \Omega_{p q}}+\frac{\partial g}{\partial H_{m n}}\left(\frac{\partial H_{m n}}{\partial \Omega_{p q}}-n^{2} \frac{\partial H_{m n}}{\partial \kappa} Y_{p q}\right)\right] \frac{\partial g}{\partial Y_{p q}}}
$$

This value of $X$ will be used to compute $D$ and the incremental damaged stress $d \sigma^{\Omega}$ with the portion $(k)$ of the strain increment that accompanied the damage process.

$$
d \sigma_{i j}^{\Omega}=k D_{i j k l} d \varepsilon_{k l}
$$

The increment of damage is found with the value of $X$ and $d \sigma^{\Omega}$ with Eqn. (2.68).

The value of $k$ has not yet been determined. To compute the part of the original stress increment that was elastic, in Figure 5.1, it can be seen that 


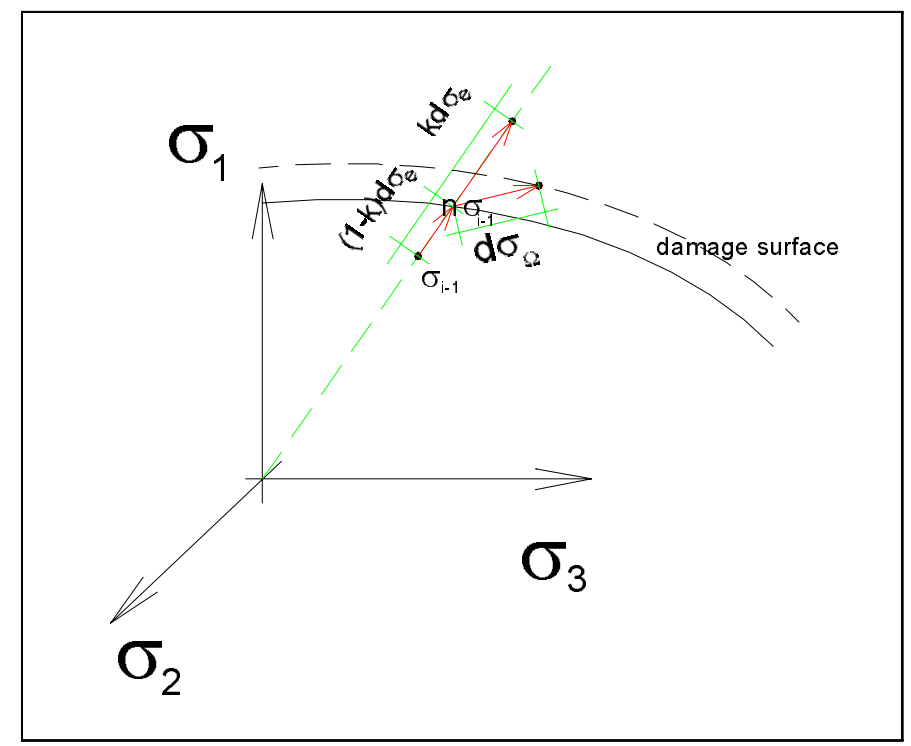

Figure 5.1: Elastic and damaged components of a stress increment.

$$
1-k=\frac{n \sigma^{i-1}-\sigma^{i-1}}{d \sigma_{e}^{i}}=(n-1) \frac{\sigma^{i-1}}{d \sigma_{e}^{i}}
$$

or

$$
k=1-(n-1) \frac{\sigma^{i-1}}{d \sigma_{e}^{i}}
$$

The above quotient between tensors is possible since they are parallel, that is, they have the same principal directions. This value of $1-k$ gives the elastic portion of the stress increment. The total and final stress increment is found with

$$
d \sigma=(1-k) d \sigma^{e}+d \sigma^{\Omega}
$$

or, taking into account Eqns. (5.1) and (5.6)

$$
d \sigma_{i j}=\left[(1-k) \hat{E}_{i j k l}+k D_{i j k l}\right] d \varepsilon_{k l}
$$




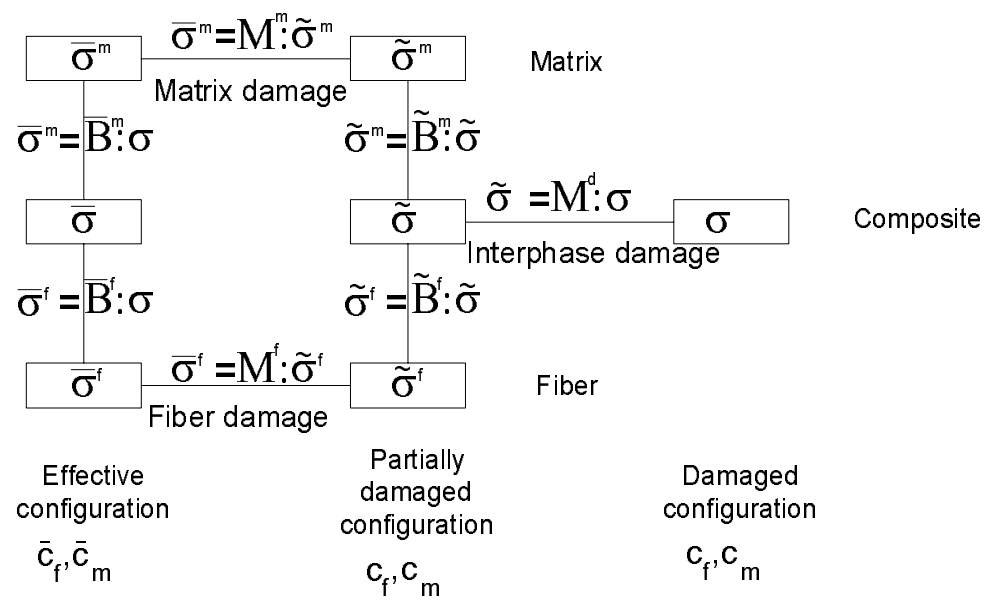

Figure 5.2: Configurations for damage computation

Finally, the nodal forces in the element are computed with

$$
f=\int_{A} B^{T} \sigma^{T} d A
$$

\subsection{Computation of damage for the composite}

At a certain state during the loading process it is assumed that the damage tensors of the three constituents and the stress and strain concentration factor in the effective and partially damaged configurations are stored in memory for all the elements and Gauss points. The increment in load produces an increment in displacement in the element. The procedure to compute the additional damage in the constituents is as follows (See Figure 5.2):

- Through classical FEM formulation the incremental strains in the Gauss points are computed.

- Since there are only three components of strain (plane strain) in the program, they are converted into 3D strains, $d \varepsilon^{(i)}$, by using the equations of plane stress.

- The vector of total strains is updated with these incremental displacements; $\varepsilon^{(i)}=$ $\varepsilon^{(i-1)}+d \varepsilon^{(i)}$ 
- It is assumed that the increment in strain will produce elastic increments of strains and stress in each constituent. The composite incremental strains in the partially damaged configuration are found with $d \tilde{\varepsilon}=M^{-d}: d \varepsilon$

- The matrix and fiber incremental strains are found with $d \tilde{\varepsilon}^{m}=A^{m}: d \tilde{\varepsilon}$, and $d \tilde{\varepsilon}^{f}=$ $A^{f}: d \tilde{\varepsilon}$.

- A loop is now performed over each constituent starting with the matrix, then the fibers and finally the composite.

For the matrix and the fibers (IPHASE=1, and IPHASE=2, procedure shown for matrix):

- Compute the elastic increment in stress with: $d \tilde{\sigma}_{e}^{m}=\tilde{E}^{m}: d \tilde{\varepsilon}^{m}$

- Compute stresses with the elastic increment: $\tilde{\sigma}_{e}^{m}=\tilde{\sigma}^{m}+d \tilde{\sigma}_{e}^{m}$

- Compute the thermodynamic force $Y_{e}^{m}$ with $\tilde{\sigma}_{e}^{m}$

- Compute value $g$ of damage surface: $g=H^{-1}: H^{-1}: Y: Y-1$

- If $g>0$ damage has occurred. Using section 5.3 compute $n$ and $X$

- If $g<0$ the material has not damaged; $X=0$

- Compute $\tilde{D}^{m}$ using Eqn. (3.32).

- Compute the damaged stress: $d \tilde{\sigma}_{\Omega}^{m}=\tilde{D}^{m}: d \tilde{\varepsilon}^{m}$

- Compute the portion $k$ of damaged stress, using Eqn. (5.7)

- Compute the increment of stress with Eqn. (5.8)

- Compute the increment of damage with Eqn. (2.68)

- Update the damage and stress tensors.

- Compute the stress in the composite due to the stress in the matrix

For the composite:

- Compute the elastic increment in stress with: $d \sigma_{e}=E: d \varepsilon$ 
- Compute stresses with the elastic increment: $\sigma_{e}=\sigma+d \sigma_{e}$

- Compute the thermodynamic force $Y_{e}$ with $\sigma_{e}$

- Compute value $g$ of damage surface: $g=H^{-1}: H^{-1}: Y: Y-1$

- If $g>0$ damage has occurred. Using section 5.3 compute $n$ and $X$

- If $g<0$ the material has not damaged; $X=0$

- Compute $D$ using Eqn. (3.76).

- Compute the damaged stress: $d \sigma_{\Omega}=D: d \varepsilon$

- Compute the portion $k$ of damaged stress, using Eqn. (5.7)

- Compute the increment of stress with Eqn. (5.8)

- Compute the increment of damage with Eqn. (2.68)

- Update the damage and stress tensors.

- Compute the nodal force using Eqn. (3.5). 


\section{Chapter 6}

\section{SUMMARY AND CONCLUSIONS}

In this work a damage model for polymer matrix composites has been introduced. The model is restricted to a single lamina with small non plastic deformations. A local analysis approach was used and the effects of damage on the matrix, fibers and composite were assembled to obtain the overall composite response.

The damage model is orthotropic and represented by a second order tensor. The matrix is isotropic; the fibers and composite orthotropic. The fiber behavior was analyzed using a statistical distribution. The evolution laws were implemented from classical dissipative theories.

Formulation for stress and strain concentration factors in damaged configurations were introduced based on the elastic concentration factors. Constitutive equations were derived for the matrix, fibers and composite. The effect of crack closure was also taken into account.

The determination of material constants was established with the use of simple experimental tests like longitudinal and transverse loading of composite specimens.

The proposed equations were implemented numerically using finite element method. Due to lack of experimental data, validation of the model is not complete and should be undertaken with additional laboratory results.

Future work may include the extension of the finite element to three dimensional case in order to include delamination damage, the establishment of a testing program to determine the material constants needed to characterize the model. ASTM standards for parameter identification is also suggested. 


\section{Appendix A}

\section{Derivatives of $M$ with respect to $\Omega$}

Paramount to the damage model presented here is the fact that $M_{i j k l}$ is related to the secondorder damage tensor $\Omega_{i j}$ through Eqn. (2.43). In the formulation of the damage evolution laws, the derivatives of $M_{i j k l}$ with respect to $\Omega_{i j}$ usually appear. These derivatives, in turn, involve the derivatives of $T_{i j}$ and $F_{i j k l}$ with respect to $\Omega_{i j}$. Looking at equation (2.37), it should be noted that the tensor $\mathbf{R}$ is a step functions, changing abruptly only when the sign of $\sigma_{i j}$ changes. But $\mathbf{R}$ remains constant. The effect is that the effective stress defined in equation (2.42) is discontinuous when the sign of $\sigma_{i j}$ changes. The discontinuity may be seen in the first derivative. When differentiating equation (2.39) and (2.25) only the derivatives of the principal values of the damage tensor will be necessary. These can be obtained by differentiating equation (2.22) with respect to the damage tensor $\Omega_{i j}$. The first differentiation yields

$$
\frac{\partial \Omega^{\prime}}{\partial \Omega_{m n}}=\frac{\frac{\partial I_{1}}{\partial \Omega_{m n}} \Omega^{\prime 2}-\frac{\partial I_{2}}{\partial \Omega_{m n}} \Omega^{\prime}+\frac{\partial I_{3}}{\partial \Omega_{m n}}}{3 \Omega^{\prime 2}-2 I_{1} \Omega^{\prime}+I_{2}}
$$

and the second differentiation 


$$
\begin{aligned}
\frac{\partial^{2} \Omega^{\prime}}{\partial \Omega_{m n} \partial \Omega_{p q}}= & {\left[\left(2 I_{1}-6 \Omega^{\prime}\right) \frac{\partial \Omega^{\prime}}{\partial \Omega_{m n}} \frac{\partial \Omega^{\prime}}{\partial \Omega_{p q}}+\Omega^{\prime 2} \frac{\partial^{2} I_{1}}{\partial \Omega_{m n} \partial \Omega_{p q}}\right.} \\
& +2 \Omega^{\prime}\left(\frac{\partial I_{1}}{\partial \Omega_{m n}} \frac{\partial \Omega^{\prime}}{\partial \Omega_{p q}}+\frac{\partial I_{1}}{\partial \Omega_{p q}} \frac{\partial \Omega^{\prime}}{\partial \Omega_{m n}}\right) \\
& -\Omega^{\prime} \frac{\partial^{2} I_{2}}{\partial \Omega_{m n} \partial \Omega_{p q}}-\left(\frac{\partial I_{2}}{\partial \Omega_{m n}} \frac{\partial \Omega^{\prime}}{\partial \Omega_{p q}}+\frac{\partial I_{2}}{\partial \Omega_{p q}} \frac{\partial \Omega^{\prime}}{\partial \Omega_{m n}}\right) \\
& \left.+\frac{\partial^{2} I_{3}}{\partial \Omega_{m n} \partial \Omega_{p q}}\right] \div\left[3 \Omega^{\prime 2}-2 I_{1} \Omega^{\prime}+I_{2}\right]
\end{aligned}
$$

By looking at Eqns (2.23) the derivatives of the invariants with respect to the damage tensor are

$$
\begin{aligned}
\frac{\partial I_{1}}{\partial \Omega_{m n}}= & \delta_{m n} \\
\frac{\partial^{2} I_{1}}{\partial \Omega_{m n} \partial \Omega_{p q}}= & 0 \\
\frac{\partial I_{2}}{\partial \Omega_{m n}}= & I_{1} \delta_{m n}-\Omega_{i j} J_{i j m n} \\
\frac{\partial^{2} I_{2}}{\partial \Omega_{m n} \partial \Omega_{p q}}= & \delta_{p q} \delta_{m n}-J_{i j p q} J_{i j m n} \\
\frac{\partial I_{3}}{\partial \Omega_{m n}}= & \varepsilon_{i j k}\left(J_{1 i m n} \Omega_{2 j} \Omega_{3 k}+J_{2 j m n} \Omega_{1 i} \Omega_{3 k}+J_{3 k m n} \Omega_{1 i} \Omega_{2 j}\right) \\
\frac{\partial^{2} I_{3}}{\partial \Omega_{m n} \partial \Omega_{p q}}= & \varepsilon_{i j k} J_{1 i m n}\left(J_{2 j p q} \Omega_{3 k}+J_{3 k p q} \Omega_{2 j}\right)+\varepsilon_{i j k} J_{2 j m n}\left(J_{1 i p q} \Omega_{3 k}+J_{3 k p q} \Omega_{1 i}\right) \\
& +\varepsilon_{i j k} J_{3 k m n}\left(J_{1 i p q} \Omega_{2 j}+J_{2 j p q} \Omega_{1 i}\right)
\end{aligned}
$$

where the constant tensor $J_{i j k l}$ is defined in Eqn. (B.17). After replacing Eqns. (A.3) in Eqns. (A.1) and (A.2) they reduce to

$$
\frac{\partial \Omega^{\prime}}{\partial \Omega_{m n}}=\frac{\Omega^{\prime 2} \delta_{m n}-\Omega^{\prime}\left(I_{1} \delta_{m n}-\Omega_{i j} J_{i j m n}\right)+\epsilon_{i j k}\left(J_{1 i m n} \Omega_{2 j} \Omega_{3 k}+J_{2 j m n} \Omega_{1 i} \Omega_{3 k}+J_{3 k m n} \Omega_{1 i} \Omega_{2 j}\right)}{3 \Omega^{\prime 2}-2 I_{1} \Omega^{\prime}+I_{2}}
$$




$$
\begin{aligned}
\frac{\partial^{2} \Omega^{\prime}}{\partial \Omega_{m n} \Omega_{p q}}= & {\left[\left(\left(2 I_{1}-6 \Omega^{\prime}\right) \frac{\partial \Omega^{\prime}}{\partial \Omega_{m n}} \frac{\partial \Omega^{\prime}}{\partial \Omega_{p q}}\right)+2 \Omega^{\prime}\left(\delta_{m n} \frac{\partial \Omega^{\prime}}{\partial \Omega_{p q}}+\delta_{p q} \frac{\partial \Omega^{\prime}}{\partial \Omega_{m n}}\right)\right.} \\
& -\Omega^{\prime}\left(\delta_{p q} \delta_{m n}-J_{i j p q} J_{i j m n}\right)-\left(I_{1} \delta_{m n}-\Omega_{i j} J_{i j m n}\right) \frac{\partial \Omega^{\prime}}{\partial \Omega_{p q}} \\
& \left.-\left(I_{1} \delta_{p q}-\Omega_{i j} J_{i j p q}\right) \frac{\partial \Omega^{\prime}}{\partial \Omega_{m n}}+\frac{\partial^{2} I_{3}}{\partial \Omega_{m n} \partial \Omega_{p q}}\right] \div\left(3 \Omega^{\prime 2}-2 I_{1} \Omega^{\prime}+I_{2}\right)
\end{aligned}
$$

Let $A_{i j k l}$ and $B_{i j k l}$ be defined as

$$
\begin{aligned}
A_{i j k l} & =\left(I_{1} \delta_{i j}-\Omega_{m n} J_{m n i j}\right) \frac{\partial \Omega^{\prime}}{\partial \Omega_{k l}} \\
B_{i j k l} & =\delta_{i j} \frac{\partial \Omega^{\prime}}{\partial \Omega_{k l}}
\end{aligned}
$$

Eqn. (A.5) becomes

$$
\begin{aligned}
\frac{\partial^{2} \Omega^{\prime}}{\partial \Omega_{m n} \Omega_{p q}}= & {\left[\left(I_{1}-6 \Omega^{\prime}\right) \frac{\partial \Omega^{\prime}}{\partial \Omega_{m n}} \frac{\partial \Omega^{\prime}}{\partial \Omega_{p q}}+4 \Omega^{\prime} \operatorname{symm}\left(B_{m n p q}\right)+\Omega^{\prime} I_{m n p q}\right.} \\
& \left.-2 \operatorname{symm}\left(A_{m n p q}\right)+\frac{\partial^{2} I_{3}}{\partial \Omega_{m n} \partial \Omega_{p q}}\right] \div\left(3 \Omega^{\prime 2}-2 I_{1} \Omega^{\prime}+I_{2}\right)
\end{aligned}
$$

Now going back to equation (2.43), and considering equations (2.37), the fourth-order damage tensor $\mathbf{M}_{i j k l}$ can be differentiated with respect to the second order damage tensor $\Omega_{i j}$, and obtain the needed equations

$$
\begin{aligned}
\frac{\partial M_{i j k l}}{\partial \Omega_{m n}}= & \left(\frac{\partial T_{i p}}{\partial \Omega_{m n}} T_{j q} F_{p q r s}+T_{i p} \frac{\partial T_{j q}}{\partial \Omega_{m n}} F_{p q r s}+T_{i p} T_{j q} \frac{\partial F_{p q r s}}{\partial \Omega_{m n}}\right) T_{r k} T_{s l} \\
& +T_{i p} T_{j q}\left(F_{p q r s} \frac{\partial T_{r k}}{\partial \Omega_{m n}} T_{s l}+F_{p q r s} T_{r k} \frac{\partial T_{s l}}{\partial \Omega_{m n}}\right)
\end{aligned}
$$

Now, the derivative $\frac{\partial T_{i j}}{\partial \Omega_{m n}}$ must be found. Since Eqn. (2.20) contains a singular tensor, only the first two equations will be differentiated together with Eqn. (2.21), to arrive at the following expressions for $\frac{\partial T_{i j}}{\partial \Omega_{m n}}$ and $\frac{\partial^{2} T_{i j}}{\partial \Omega_{m n} \partial \Omega_{p q}}$ 


$$
\frac{\partial T_{i j}}{\partial \Omega_{k l}}=P_{j m}^{-1} \frac{\partial L_{m n}}{\partial \Omega_{k l}} T_{i n}
$$

and

$$
\frac{\partial^{2} T_{i j}}{\partial \Omega_{m n} \partial \Omega_{p q}}=P_{j s}^{-1}\left(\frac{\partial^{2} L_{s k}}{\partial \Omega_{m n} \partial \Omega_{p q}} T_{i k}+\frac{\partial L_{s k}}{\partial \Omega_{m n}} \frac{\partial T_{i k}}{\partial \Omega_{p q}}-\frac{\partial P_{s k}}{\partial \Omega_{m n}} \frac{\partial T_{i k}}{\partial \Omega_{p q}}\right)
$$

with

$$
\begin{aligned}
P & =\left[\begin{array}{ccc}
\Omega_{11}-\Omega_{q} & \Omega_{12} & \Omega_{13} \\
\Omega_{21} & \Omega_{22}-\Omega_{q} & \Omega_{23} \\
T_{q 1} & T_{q 2} & T_{q 3}
\end{array}\right] \\
L= & {\left[\begin{array}{ccc}
\Omega_{11}-\Omega_{q} & \Omega_{12} & \Omega_{13} \\
\Omega_{21} & \Omega_{22}-\Omega_{q} & \Omega_{23} \\
0 & 0 & 0
\end{array}\right] }
\end{aligned}
$$

The index $q$ above corresponds to the first index of $T_{i j}$. That means there is a $P$ tensor for each eigenvector.

Also the derivatives of $F_{i j k l}$ with respect to $\Omega_{m n}$ must be calculated. By differentiating Eqn. (2.39) twice, the following expressions are obtained

$$
\begin{aligned}
\frac{\partial F_{i j k l}}{\partial \Omega_{m n}}= & {\left[\frac{\zeta_{i j k l}\left\langle\sigma_{k l}^{\prime}\right\rangle}{\left(1-\Omega_{i j}^{\prime}\right)^{2}}+\frac{C_{n} \zeta_{i j k l}\left\langle-\sigma_{k l}^{\prime}\right\rangle}{\left(1-C_{n} \Omega_{i j}^{\prime}\right)^{2}}\right] \frac{\partial \Omega_{i j}^{\prime}}{\partial \Omega_{m n}} } \\
& +\frac{1}{2} C_{s}\left(I_{i j k l}-\zeta_{i j k l}\right)\left[\frac{1}{\left(1-C_{s} \Omega_{i i}^{\prime}\right)^{2}} \frac{\partial \Omega_{i i}^{\prime}}{\partial \Omega_{m n}}+\frac{1}{\left(1-C_{s} \Omega_{j j}^{\prime}\right)^{2}} \frac{\partial \Omega_{j j}^{\prime}}{\partial \Omega_{m n}}\right]
\end{aligned}
$$




$$
\begin{aligned}
\frac{\partial^{2} F_{i j k l}}{\partial \Omega_{m n} \partial \Omega_{p q}}= & {\left[\frac{2 \zeta_{i j k l}\left\langle\sigma_{k l}^{\prime}\right\rangle}{\left(1-\Omega_{i j}^{\prime}\right)^{3}}+\frac{2 C_{n}^{2} \zeta_{i j k l}\left\langle-\sigma_{k l}^{\prime}\right\rangle}{\left(1-C_{n} \Omega_{i j}^{\prime}\right)^{3}}\right] \frac{\partial \Omega_{i j}^{\prime}}{\partial \Omega_{p q}} \frac{\partial \Omega_{i j}^{\prime}}{\partial \Omega_{m n}} } \\
& +\left[\frac{\zeta_{i j k l}\left\langle\sigma_{k l}^{\prime}\right\rangle}{\left(1-\Omega_{i j}^{\prime}\right)^{2}}+\frac{C_{n} \zeta_{i j k l}\left\langle-\sigma_{k l}^{\prime}\right\rangle}{\left(1-C_{n} \Omega_{i j}^{\prime}\right)^{2}}\right] \frac{\partial^{2} \Omega_{i j}^{\prime}}{\partial \Omega_{m n} \partial \Omega_{p q}} \\
& +\frac{C_{s}^{2}\left(I_{i j k l}-\zeta_{i j k l}\right)}{\left(1-C_{s} \Omega_{i i}^{\prime}\right)^{3}} \frac{\partial \Omega_{i i}^{\prime}}{\partial \Omega_{p q}} \frac{\partial \Omega_{i i}^{\prime}}{\partial \Omega_{m n}}+\frac{1}{2} \frac{C_{s}\left(I_{i j k l}-\zeta_{i j k l}\right)}{\left(1-C_{s} \Omega_{i i}^{\prime}\right)^{2}} \frac{\partial^{2} \Omega_{i i}^{\prime}}{\partial \Omega_{m n} \partial \Omega_{p q}} \\
& +\frac{C_{s}^{2}\left(I_{i j k l}-\zeta_{i j k l}\right)}{\left(1-C_{s} \Omega_{j j}^{\prime}\right)^{3}} \frac{\partial \Omega_{j j}^{\prime}}{\partial \Omega_{p q}} \frac{\partial \Omega_{j j}^{\prime}}{\partial \Omega_{m n}}+\frac{1}{2} \frac{C_{s}\left(I_{i j k l}-\zeta_{i j k l}\right)}{\left(1-C_{s} \Omega_{j j}^{\prime}\right)^{2}} \frac{\partial^{2} \Omega_{j j}^{\prime}}{\partial \Omega_{m n} \partial \Omega_{p q}}
\end{aligned}
$$

Another derivative that will prove to be helpful in the model is $\partial M_{i j k l}^{-1} / \partial \Omega_{m n}$. To find it, from Eqn. (2.43), this expression can be written

$$
I_{r s k l}=M_{r s i j}^{-1} T_{i m} T_{j n} F_{m n p q} T_{p k} T_{q l}
$$

Once it is differentiated with respect to $\Omega_{m n}$ and solved for $\partial M_{i j k l}^{-1} / \partial \Omega_{m n}$

$$
\begin{aligned}
\frac{\partial M_{i j k l}^{-1}}{\partial \Omega_{m n}}= & -M_{i j r s}^{-1} W_{c d k l}^{-1}\left[\left(\frac{\partial T_{r c}}{\partial \Omega_{m n}} T_{s d}+\frac{\partial T_{r d}}{\partial \Omega_{m n}} T_{s c}\right)+\left(T_{r c} \frac{\partial T_{s d}}{\partial \Omega_{m n}}+T_{r d} \frac{\partial T_{s c}}{\partial \Omega_{m n}}\right)\right. \\
& +2 T_{r t} T_{s u} \frac{\partial F_{t u b a}}{\partial \Omega_{m n}} F_{b a c d}^{-1}+2 T_{r t} T_{s u} F_{t u p a} \frac{\partial T_{p q}}{\partial \Omega_{m n}} T_{q b}^{-1} F_{b a c d}^{-1} \\
& \left.+2 T_{r t} T_{s u} F_{t u b p} \frac{\partial T_{p q}}{\partial \Omega_{m n}} T_{q a}^{-1} F_{b a c d}^{-1}\right]
\end{aligned}
$$

with

$$
W_{i j k l}=T_{i k} T_{j l}+T_{i l} T_{j k}
$$




\section{Appendix B}

\section{Tensor Algebra}

In the derivation of many of the equations in this dissertation, many tensorial operations were needed. Since most of these operations are not easily found in textbooks, they are presented here for reference.

\section{B.1 Tensor symmetries}

There are two kinds of symmetries in a fourth-order tensor. A tensor $A_{i j k l}$ has a minor symmetry when

$$
A_{i j k l}=A_{j i k l}=A_{i j l k}
$$

The same tensor has a major symmetry when

$$
A_{i j k l}=A_{k l i j}
$$

\section{B.2 Matrix representation of a tensor}

A tensor $A_{i j k l}$ with a minor symmetry has only 36 independent constants. Therefore it can be represented by a $6 x 6$ matrix called reduced form. Let small cap $[a]$ be the reduced form of the tensor capital $A$. The reduced form is used in the computer program to save storage 
and time. Each element of $[a]$ corresponds to an element in the tensor $A_{i j k l}$ according to the following transformation:

$$
a_{\alpha \beta} \rightarrow A_{i j k l}
$$

with:

$$
\begin{aligned}
& \alpha=i \text { when } i=j \\
& \alpha=9-(i+j) \text { when } i \neq j
\end{aligned}
$$

The same transformations apply between $\beta$ and $k$ and $l$.

In matrix representation, the above mapping can be shown as:

$$
[a]=\left[\begin{array}{cccccc}
A_{1111} & A_{1122} & A_{1133} & A_{1123} & A_{1113} & A_{1112} \\
A_{2211} & A_{2222} & A_{2233} & A_{2223} & A_{2213} & A_{2212} \\
A_{3311} & A_{3322} & A_{3333} & A_{3323} & A_{3313} & A_{3312} \\
A_{2311} & A_{2322} & A_{2333} & A_{2323} & A_{2313} & A_{2312} \\
A_{1311} & A_{1322} & A_{1333} & A_{1323} & A_{1313} & A_{1312} \\
A_{1211} & A_{1222} & A_{1233} & A_{1223} & A_{1213} & A_{1212}
\end{array}\right]
$$

It would be convenient to perform the usual tensor operations using the $6 \times 6$ matrices representing them, especially if the result can, in turn, be represented by another reduced form. This will save additional memory and time since it is faster to operate on 36 elements than on 81 elements. Examples of these operations are the inner product of two fourth-order tensors and the inverse of a fourth-order tensor. However, care should be taken because there cannot be a direct exchange of indexes in the transformed matrices in performing those operations. As an example, if $A_{i j k l}$ and $B_{i j k l}$ are fourth-order tensors and $C_{i j k l}$ is the result of the following tensor multiplication

$$
\begin{aligned}
\mathbf{C} & =\mathbf{A}: \mathbf{B} \\
C_{i j k l} & =A_{i j m n} B_{m n k l}
\end{aligned}
$$


and let $[a],[b]$, and $[c]$ the reduced $6 \mathrm{x} 6$ matrix representations of the above tensors, it is verified that

$$
\begin{aligned}
{[a][b] } & \neq[c] \text { or } \\
a_{\alpha \beta} b_{\beta \gamma} & \neq c_{\alpha \gamma} \text { (matrix multiplication) }
\end{aligned}
$$

Anyway, the reduced forms can still be used if the rules set forth in the next sections are followed.

\section{B.3 Inner-product tensor multiplication}

Taking the example of the previous section, an element like $C_{1211}$ has the following value

$$
\begin{aligned}
C_{1211}= & A_{1211} B_{1111}+A_{1222} B_{2211}+A_{1233} B_{3311} \\
& +2 A_{1212} B_{1211}+2 A_{1213} B_{1311}+2 A_{1223} B_{2311}
\end{aligned}
$$

One way to get this result by performing a matrix multiplication is to multiply the last three columns of the matrix $[a]$ by 2 , and then perform the multiplication

$$
\begin{aligned}
& {[c]=\left[\begin{array}{cccccc}
A_{1111} & A_{1122} & A_{1133} & 2 A_{1123} & 2 A_{1113} & 2 A_{1112} \\
A_{2211} & A_{2222} & A_{2233} & 2 A_{2223} & 2 A_{2213} & 2 A_{2212} \\
A_{3311} & A_{3322} & A_{3333} & 2 A_{3323} & 2 A_{3313} & 2 A_{3312} \\
A_{2311} & A_{2322} & A_{2333} & 2 A_{2323} & 2 A_{2313} & 2 A_{2312} \\
A_{1311} & A_{1322} & A_{1333} & 2 A_{1323} & 2 A_{1313} & 2 A_{1312} \\
A_{1211} & A_{1222} & A_{1233} & 2 A_{1223} & 2 A_{1213} & 2 A_{1212}
\end{array}\right]} \\
& {\left[\begin{array}{cccccc}
B_{1111} & B_{1122} & B_{1133} & B_{1123} & B_{1113} & B_{1112} \\
B_{2211} & B_{2222} & B_{2233} & B_{2223} & B_{2213} & B_{2212} \\
B_{3311} & B_{3322} & B_{3333} & B_{3323} & B_{3313} & B_{3312} \\
B_{2311} & B_{2322} & B_{2333} & B_{2323} & B_{2313} & B_{2312} \\
B_{1311} & B_{1322} & B_{1333} & B_{1323} & B_{1313} & B_{1312} \\
B_{1211} & B_{1222} & B_{1233} & B_{1223} & B_{1213} & B_{1212}
\end{array}\right]}
\end{aligned}
$$


This transformation can be produced by using the transformation matrix $R$

$$
[R]=\left[\begin{array}{llllll}
1 & 0 & 0 & 0 & 0 & 0 \\
0 & 1 & 0 & 0 & 0 & 0 \\
0 & 0 & 1 & 0 & 0 & 0 \\
0 & 0 & 0 & 2 & 0 & 0 \\
0 & 0 & 0 & 0 & 2 & 0 \\
0 & 0 & 0 & 0 & 0 & 2
\end{array}\right]
$$

Substituting this matrix in Eqn. (B.6)

$$
[c]=[a][R][b]
$$

\section{B.4 Tensor inversion}

It is convenient to first define the fourth-order identity tensor. The fourth-order identity tensor $I_{i j k l}$ is a tensor that multiplied innerly by another fourth-order tensor yields this same tensor, that is

$$
I_{i j m n} A_{m n k l}=A_{i j k l}
$$

If $A_{i j k l}$ has a minor symmetry, the tensor that complies with the above definition is

$$
I_{i j k l}=\frac{1}{2}\left(\delta_{i k} \delta_{j l}+\delta_{i l} \delta_{j k}\right)
$$

where $\delta_{i j}$ is the Kronecker delta, defined as

$$
\begin{array}{ll}
\delta_{i j}=1 & \text { if } i=j \\
\delta_{i j}=0 & \text { if } i \neq j
\end{array}
$$

In the reduced form, the matrix $[i]$ is therefore 


$$
[i]=\left[\begin{array}{cccccc}
1 & 0 & 0 & 0 & 0 & 0 \\
0 & 1 & 0 & 0 & 0 & 0 \\
0 & 0 & 1 & 0 & 0 & 0 \\
0 & 0 & 0 & 1 / 2 & 0 & 0 \\
0 & 0 & 0 & 0 & 1 / 2 & 0 \\
0 & 0 & 0 & 0 & 0 & 1 / 2
\end{array}\right]=[R]^{-1}
$$

With the identity tensor already defined, the inverse of a tensor is defined as another tensor that multiplied innerly by the original tensor yields the identity tensor. That is

$$
A_{i j m n} A_{m n k l}^{-1}=I_{i j k l}
$$

The following notation is introduced:

$[a]^{-1}=$ inverse of the reduced form of $A_{i j k l}$

$\left[a^{-1}\right]=$ reduced form of the inverse of $A_{i j k l}$

If $A_{i j k l}$ has a minor symmetry, the procedure to find the components of $a_{\alpha \beta}^{-1}$ is:

1. Multiply the last three columns of $[a]$ by 2 by using the matrix $[R]$

2. Invert the obtained matrix.

3. Multiply the matrix by $[i]$

In order words, the matrix $[a]^{-1}$ is computed as:

$$
\left[a^{-1}\right]=[[a][R]]^{-1}[i]=[i][a]^{-1}[i]
$$




\section{B.5 Tensor differentiation}

\section{B.5.1 Derivative of a tensor with respect to itself}

For any symmetric second order tensor $\Phi_{i j}$, it is verified:

$$
d \Phi_{i j}=d \Phi_{j i}
$$

This leads to the following equation for differentiating a second-order symmetric tensor with respect to itself:

$$
\frac{\partial \Phi_{i j}}{\partial \Phi_{k l}}=J_{i j k l}
$$

where $J_{i j k l}$ is a fourth-order tensor defined as:

$$
\begin{array}{cc}
J_{i j k l}=1 & \text { if } i=k, \text { and } j=l \\
J_{i j k l}=1 & \text { if } i=l, \text { and } j=k \\
J_{i j k l}=0 & \text { otherwise }
\end{array}
$$

In its reduced form, the tensor $J_{i j k l}$ is represented as:

$$
[j]=\left[\begin{array}{llllll}
1 & 0 & 0 & 0 & 0 & 0 \\
0 & 1 & 0 & 0 & 0 & 0 \\
0 & 0 & 1 & 0 & 0 & 0 \\
0 & 0 & 0 & 1 & 0 & 0 \\
0 & 0 & 0 & 0 & 1 & 0 \\
0 & 0 & 0 & 0 & 0 & 1
\end{array}\right]
$$

\section{B.5.2 Derivative of the inverse of a tensor with respect to the tensor}

Starting with a property of the Kronecker $\delta$ : 


$$
A_{i j} A_{j k}^{-1}=\delta_{i k}
$$

Differentiating Eqn. (B.19) with respect to $A_{m n}$ and rearranging terms:

$$
A_{i j} \frac{\partial A_{j k}^{-1}}{\partial A_{m n}}=-\frac{\partial A_{i j}}{\partial A_{m n}} A_{j k}^{-1}
$$

Pre-multiplying both sides by $A_{l i}^{-1}$ and rearranging again:

$$
\frac{\partial A_{i j}^{-1}}{\partial A_{m n}}=-A_{i k}^{-1} \frac{\partial A_{k l}}{\partial A_{m n}} A_{l j}^{-1}
$$

And because of Eqn. (B.16):

$$
\frac{\partial A_{i j}^{-1}}{\partial A_{m n}}=-A_{i k}^{-1} J_{k l m n} A_{l j}^{-1}
$$




\section{Appendix $\mathbf{C}$}

\section{Minimum square of type $y=a[f(x)]^{b}$}

The data fit proposed in Section 4.6 requires a function of the type $y=a[f(x)]^{b}$. This case is not frequently covered in data-fitting cases. The equations needed to solve the problem are presented here. They are based on minimization of the summation of the squares of differences.

The difference between the given function and the experimental data for one point is:

$$
\Delta=a\left[f\left(x_{i}\right)\right]^{b}-y_{i}
$$

The square of this difference is:

$$
\Delta^{2}=a^{2}\left[f\left(x_{i}\right)\right]^{2 b}-2 a\left[f\left(x_{i}\right)\right]^{b} y_{i}+y_{i}^{2}
$$

The summation for all the points is:

$$
S=\sum \Delta^{2}=a^{2} \sum\left[f\left(x_{i}\right)\right]^{2 b}-2 a \sum\left[f\left(x_{i}\right)\right]^{b} y_{i}+\sum y_{i}^{2}
$$

To minimize this summation, the function $S$ is differentiated respect to $a$ and $b$ :

$$
\begin{aligned}
& \frac{\partial S}{\partial a}=a \sum\left[f\left(x_{i}\right)\right]^{2 b}-\sum\left[f\left(x_{i}\right)\right]^{b} y_{i}=0 \\
& \frac{\partial S}{\partial b}=a \sum\left[f\left(x_{i}\right)\right]^{2 b} \ln f\left(x_{i}\right)-\sum\left[f\left(x_{i}\right)\right]^{b} \ln f\left(x_{i}\right) y_{i}=0
\end{aligned}
$$


From Eqn. (C.4) and (C.5):

$$
\sum\left[f\left(x_{i}\right)\right]^{b} y_{i} \sum\left[f\left(x_{i}\right)\right]^{2 b} \ln f\left(x_{i}\right)-\sum\left[f\left(x_{i}\right)\right]^{2 b} \sum\left[f\left(x_{i}\right)\right]^{b} \ln f\left(x_{i}\right) y_{i}=0
$$

The transcendental equation (C.6) yields the value of $b$. It can be solved by trial and error. The value of the constant $a$ is found from Eqn. (C.4):

$$
a=\frac{\sum\left[f\left(x_{i}\right)\right]^{b} y_{i}}{\sum\left[f\left(x_{i}\right)\right]^{2 b}}
$$

If the resulting equation must pass through a certain point $\left(x_{0}, y_{0}\right)$ the following equation must be verified

$$
y_{0}=a\left[f\left(x_{0}\right)\right]^{b}
$$

After replacing with Eqn. (C.8) the summation of Eqn. (C.3) becomes

$$
S=y_{0}^{2} \sum\left[\frac{f\left(x_{i}\right)}{f\left(x_{0}\right)}\right]^{2 b}-2 y_{0} \sum\left[\frac{f\left(x_{i}\right)}{f\left(x_{0}\right)}\right]^{b} y_{i}+\sum y_{i}^{2}
$$

And differentiating with respect to $b$ to minimize

$$
\frac{\partial S}{\partial b}=y_{0} \sum\left[\frac{f\left(x_{i}\right)}{f\left(x_{0}\right)}\right]^{2 b} \ln \left[\frac{f\left(x_{i}\right)}{f\left(x_{0}\right)}\right]-\sum\left[\frac{f\left(x_{i}\right)}{f\left(x_{0}\right)}\right]^{b} y_{i} \ln \left[\frac{f\left(x_{i}\right)}{f\left(x_{0}\right)}\right]=0
$$

which, again, must be solved by trial and error 


\section{Appendix D}

\section{Elastic stress and strain concentration factors}

The following closed-form formulation for the elastic stress and strain concentration factors has been taken from Luciano and Barbero [14].

The stress concentration factor for the fibers is given by

$$
\bar{B}_{i j k l}^{f}=\left[I_{i j k l}+c^{m} \bar{E}_{i j m n}^{m}\left(I_{m n p q}-\frac{P_{m n r s} \bar{E}_{r s p q}^{m}}{c^{m}}\right)\left(\bar{E}_{p q k l}^{-f}-\bar{E}_{p q k l}^{-m}\right)\right]^{-1}
$$

The tensor $P$ is defined as

$$
P_{i j k l}=\frac{1}{\mu_{0}} \sum_{\xi}^{ \pm \infty} t(\xi)\left[g_{i j k l}^{(1)}-\frac{1}{1-\nu} g_{i j k l}^{(2)}+\frac{v}{1-\nu} g_{i j k l}^{(3)}\right]
$$

with

$$
\begin{gathered}
g_{i j k l}^{(1)}=\frac{1}{2 \xi^{2}}\left[\xi_{j}\left(\delta_{i l} \xi_{k}+\delta_{i k} \xi_{l}\right)+\xi_{i}\left(\delta_{j l} \xi_{k}+\delta_{j k} \xi_{l}\right)\right] \\
g_{i j k l}^{(2)}=\frac{\xi_{i} \xi_{j} \xi_{k} \xi_{l}}{\xi^{4}}
\end{gathered}
$$




$$
g_{i j k l}^{(3)}=\frac{\xi_{i} \xi_{j} \delta_{k l}}{\xi^{2}}
$$

and

$$
\xi^{2}=\xi_{i} \xi_{i}
$$

The following series are now defined

$$
\begin{aligned}
& S_{1}=\sum_{\xi}^{ \pm \infty} t(\xi) \bar{\xi}_{1}^{2} \\
& S_{2}=\sum_{\xi}^{ \pm \infty} t(\xi) \bar{\xi}_{2}^{2} \\
& S_{3}=\sum_{\xi}^{ \pm \infty} t(\xi) \bar{\xi}_{3}^{2} \\
& S_{4}=\sum_{\xi}^{ \pm \infty} t(\xi) \bar{\xi}_{1}^{4} \\
& S_{5}=\sum_{\xi}^{ \pm \infty} t(\xi) \bar{\xi}_{2}^{4} \\
& S_{6}=\sum_{\xi}^{ \pm \infty} t(\xi) \bar{\xi}_{3}^{4} \\
& S_{7}=\sum_{\xi}^{ \pm \infty} t(\xi) \bar{\xi}_{2}^{2} \bar{\xi}_{3}^{2} \\
& S_{9}=\sum_{\xi}^{ \pm \infty} t(\xi) \bar{\xi}_{1}^{2} \bar{\xi}_{2}^{2} \\
& S_{8}=\sum_{\xi} t(\xi) \bar{\xi}_{1}^{2} \bar{\xi}_{3}^{2} \\
& \pm \infty
\end{aligned}
$$

with 


$$
\bar{\xi}_{i}=\frac{\xi_{i}}{\xi}
$$

In terms of the series $S_{i}$ the tensor $P$ can be expressed in its reduced form (6x6 matrix) as

$$
[p]=\left[\begin{array}{ccc}
\frac{2 S_{1}}{\mu_{m}}-\frac{S_{4}}{2 \mu_{m}\left(1-\nu_{m}\right)} & -\frac{S_{9}}{2 \mu_{m}\left(1-\nu_{m}\right)} & -\frac{S_{8}}{2 \mu_{m}\left(1-\nu_{m}\right)} \\
\text { symm } & \frac{2 S_{2}}{\mu_{m}}-\frac{S_{5}}{2 \mu_{m}\left(1-\nu_{m}\right)} & -\frac{S_{7}}{2 \mu_{m}\left(1-\nu_{m}\right)} \\
\text { symm } & \text { symm } & \frac{2 S_{3}}{\mu_{m}}-\frac{S_{6}}{2 \mu_{m}\left(1-\nu_{m}\right)} \\
\text { symm } & \text { symm } & \text { symm } \\
\text { symm } & \text { symm } & \text { symm } \\
\text { symm } & \text { symm } & \text { symm } \\
0 & 0 & 0 \\
0 & 0 & 0 \\
0 & 0 & 0 \\
\frac{S_{7}}{2 \mu_{m}\left(1-\nu_{m}\right)} & \frac{S_{1}+S_{3}}{2 \mu_{m}}-\frac{S_{8}}{2 \mu_{m}\left(1-\nu_{m}\right)} & 0 \\
\text { symm } & \text { symm } & \frac{S_{1}+S_{2}}{2 \mu_{m}}-\frac{S_{9}}{2 \mu_{m}\left(1-\nu_{m}\right)}
\end{array}\right]
$$

For unidirectional composites with long circular cylindrical fibers the following expressions apply

$$
\begin{aligned}
& S_{1}=S_{4}=S_{8}=S_{9}=0 \\
& S_{2}=S_{3} \\
& S_{5}=S_{6}
\end{aligned}
$$

The series $S_{3}, S_{6}$, and $S_{7}$ can be expressed with these parabolic expressions

$$
\begin{aligned}
& S_{3}=0.49247-0.47603 c_{f}-0.02748 c_{f}^{2} \\
& S_{6}=0.36844-0.14944 c_{f}-0.27152 c_{f}^{2} \\
& S_{7}=0.12346-0.32035 c_{f}+0.23517 c_{f}^{2}
\end{aligned}
$$


The stress concentration factor for the matrix can now be found with:

$$
c^{m} \bar{B}_{i j k l}^{m}+c^{f} \bar{B}_{i j k l}^{f}=I_{i j k l}
$$

Now for the strain concentration factor for the fiber this following equation applies

$$
\bar{A}_{i j k l}^{f}=\left[I_{i j k l}+P_{i j m n}\left(\bar{E}_{m n k l}^{f}-\bar{E}_{m n k l}^{m}\right)\right]^{-1}
$$

and the strain concentration for the matrix can be found with

$$
c^{m} \bar{A}_{i j k l}^{m}+c^{f} \bar{A}_{i j k l}^{f}=I_{i j k l}^{f}
$$




\section{Bibliography}

[1] KACHANOV, L. M., "On the Time to Failure under Creep Conditions", Izv, AN SSSR, Otd. Tekhn. Nauk, No. 8, pp. 26-31, (1958)

[2] RABOTNOV, Y. N., "Creep Rupture", in Proceedings XII International Congress on Applied Mechanics, Stanford, Springer, Berlin, (1968)

[3] LEMAitRE, J., and ChABOCHE, J. L., " A Non-linear Model of Creep-Fatigue Damage Cumulation and Interaction", Proceedings IUTAM Symposium on Mechanics of Visco-elastic Media and Bodies, Springer, Gothenburg, (1974)

[4] HULT, J., On Topics in Applied Continuum Mechanics, (J. L. Zeman and F. Ziegler, eds.), Springer, New York, (1974)

[5] LECKIE, F. A., "The Constitutive Equations of Continuum Creep-Fatigue Damage Cumulation and Interaction, Philosophical Transactions Royal Society of London, vol. A288, pp. 27, (1978)

[6] MURAKAMI S., "Notion of Continuum Damage Mechanics and its Applications to Anisotropic Creep Damage Theory", Journal of Engineering Materials Technology, vol. 105, pp. 99-105 (1983)

[7] VOYIADJIS, G. Z., and PARK, T., "Local and Interfacial Damage Analysis of Metal Matrix Composites", International Journal of Engineering Sciences, vol. 33, No. 11, pp. $1595-1621,(1995)$

[8] MROZ, Z., "Mathematical Models of Inelastic Material Behavior", University of Waterloo, pp. 120-146, (1973)

[9] KACHANOV, L.M., "Introduction to Continuum Damage Mechanics", Martinus Nijhoff, Dordrecht, The Netherlands, (1986) 
[10] Lemaitre, J. and CHABOCHE, J. L., "Mechanics of Solid Materials", Cambridge University Press, (1990)

[11] HAYHURST, D. R., "Creep Rupture under Multiaxial State of Stress," Journal of Mechanics and Physics of Solids, vol. 29, No. 6, pp. 381-390, (1972)

[12] ARNOLD, S. M. and KRUCH S., "Differential Continuum Damage Mechanics Models for Creep and Fatigue of Unidirectional Metal Matrix Composites," NASA Technical Memorandum TM-105213, (1991)

[13] MURAKAMI, S., "Mechanical Modeling of Material Damage," Transactions of the ASME, vol. 55, pp. 280-286. (1988)

[14] LUCIANO, R. and BARBERO, E. J. "Formulas for the Stiffness of Composites with Periodic Microstructure," International Journal of Solids and Structures, vol. 31, No. 21, pp. 2933-2944, (1994)

[15] TAlrejA, R., "A Continuum Mechanics Characterization of Damage in Composite Materials," Proceedings Royal Society of London, A399, 195, (1985)

[16] CHRISTENSEN, R. M., "Tensor Transformation and Failure Criteria for the Analysis of Composite Materials," Journal of Composite Materials, 22, 874, (1988)

[17] HILL, R., "A Self-Consistent Mechanics of Composite Materials," Journal of the Mechanics and Physics of Solids, 13, 213, (1965)

[18] DVORAK, G. J., and BAHEI-EL-DIN, Y. A., "Elastic-Plastic Behavior of Fibrous Composites," Journal of Mechanics and Physics of Solids, 48, 809, (1979)

[19] NEEDLEMAN, A., "A continuum model for void nucleation by inclusion debonding," Journal of Applied Mechanics, Vol. 54, pp. 525-531, (1987)

[20] TVERGAARD, V., "Effect of fibre debonding in a whisker-reinforced metal." Materials Science and Engineering, Vol. A125, pp. 203-213, (1990)

[21] ABOUDI, J., "Constitutive Equations for Elastoplastic Composites with Imperfect Bonding," International Journal of Plasticity, Vol. 4, pp. 103-125, (1988)

[22] TEPLY, J. L., and DVORAK, G. J., "Dual Estimates of Overall Instantaneous Properties of Elasto-Plastic Composites," 5th International Symposium on Continuum Models of Discrete System, Nottingham, July 14-20, pp. 205-216, (1985) 
[23] Christman, T., NEEdleman, A., NUTT, S., and SURESH, S., "On the Microstructural Evolution and Micromechanical Modeling of Deformation of a Whisker Reinforced Metal Matrix Composite," Materials Science and Engineering A, Vol. 107, pp. 49-61, (1989)

[24] SHERWOOD, J. A., and BOYLE, M. J., "Investigation of the Thermomechanical Response of a Titanium-Aluminide/Silicon-Carbide Composite Using a Unified State Variable Model and the Finite Element Method," Microcracking-Induced Damage in Composites, AMD-Vol. 111., G. J. Dvorak and D. C. Lagoudas, eds. American Society of Mechanical Engineers, pp. 151-161, (1990)

[25] BARBERO, E., and GANGARAO, H. V. S., "Structural Applications of Composites in Infrastructure. Part I," SAMPE Journal, Vol. 27, No. 6, pp. 9-16, (1991)

[26] BARBERO, E., and GANGARAO, H. V. S., "Structural Applications of Composites in Infrastructure. Part II," SAMPE Journal, Vol. 28, No. 1, pp. 9-16, (1992)

[27] DAVIDSON, L., and STEVENS, A. L., "Thermomechanical constitution of spalling elastic bodies," Journal of Applied Physics, Vol. 44, pp. 667-674, (1973)

[28] KRAJCINOVIC, D., and FONSEKA, G. U., "The Continuous Damage Theory of Brittle Materials," Journal of Applied Mechanics, Vol. 48, pp. 809-824, (1981)

[29] OWEN, D. R. J., and HINTON E., "Finite Element in Plasticity: Theory and Practice," Pineridge Press Limited, pp. 249-253, (1980)

[30] STUMVOLL, M. and SWOBODA, G., "Deformation Behavior of Ductile Solids Containing Anisotropic Damage," Journal of Engineering Mechanics, Vol. 119, No. 7, pp. 1331-1352, (1993).

[31] WENG, G., International Journal of Engineering Sciences, Vol. 22, pp. 845, (1984)

[32] KELLY, K. W., and BARBERO, E., "The Effect of Fiber Damage on the Longitudinal Creep of a CFMMC," International Journal of Solid Structures, Vol. 30, No. 24, pp. $3417-3429,(1993)$

[33] ROSEn, B. W., "Tensile Failure of Fibrous Composites," AIAA Journal, Vol. 2, No. $11,(1964)$

[34] CHRISFIELD, M. A., "Non-linear Finite Element Analysis of Solids and Structures," John Wiley \& Sons 
[35] LEMAITRE, J., " A Course in Damage Mechanics," Springer-Verlag, (1992)

[36] JANSON, J., and HULT, J., "Damage Mechanics and Fracture Mechanics - A Combined Approach," Journal de Mechanique Applique, Vol 1, pp.69-84, (1977)

[37] BARBERO, E., "Introduction to Composite Materials Design," Taylor \& Francis, (1999)

[38] IWAKUMA, T., and NEMAT-NASSER, S., "Composites with Periodic Microstructure," Computers and Structures, Vol. 16, No. 1-4, pp. 13-19, (1983) 


\title{
LOCAL DAMAGE ANALYSIS OF FIBER REINFORCED POLYMER MATRIX COMPOSITES
}

\author{
by \\ Arsenio Caceres \\ A DISSERTATION \\ Submitted to \\ West Virginia University \\ in partial fulfillment of the requirements \\ for the degree of \\ Doctor of Philosophy \\ APPROVAL OF EXAMINING COMMITTEE
}

Dr. Carl Irwin

Dr. Tim Norman

Dr. Nithi Sivaneri

Dr. Julio Davalos

Date

Dr. Ever Barbero, Chairman 